\title{
Focal Spinal Nondisjunction in Primary Neurulation : Limited Dorsal Myeloschisis and Congenital Spinal Dermal Sinus Tract
}

\author{
Sui-To Wong, ${ }^{1}$ Dachling Pang ${ }^{2,3}$ \\ Department of Neurosurgery, Tuen Mun Hospital, Hong Kong, Hong Kong \\ Department of Paediatric Neurosurgery, ${ }^{2}$ University of California, Davis, CA, USA \\ Department of Paediatric Neurosurgery, ${ }^{3}$ Great Ormond Street Hospital for Children, NHS Trust, London, UK
}

Spinal dysraphic lesions due to focal nondisjunction in primary neurulation are commonly encountered in paediatric neurosurgery, but the "fog-of-war" on these conditions was only gradually dispersed in the past 10 years by the works of the groups led by the senior author and Prof. Kyu-Chang Wang. It is now clear that limited dorsal myeloschisis and congenital spinal dermal sinus tract are conditions at the two ends of a spectrum; and mixed lesions of them with various configurations exist. This review article summarizes the current understanding of these conditions' embryogenetic mechanisms, pathological anatomy and clinical manifestations, and their management strategy and surgical techniques.

Key Words : Limited dorsal myeloschisis · Spinal dermal sinus tract · Dermoid · Nondisjunction · Dysraphism · Focal spinal nondisjunctional disorders.

\section{INTRODUCTION}

Focal nondisjunction of the primary neural tube in its spinal cord region results in a normal or near-normal spinal cord, except for the presence of a tract anchoring the dorsal surface of the spinal cord to the base of a characteristic skin lesion. This tract, depending on its cellular constituents and its completeness in extent, can lead to tethered cord syndrome and/or collection of dermal sinus tissue with their dreadful effects. This review aims to give a concise and updated summary of the conditions that have been grouped under a common embryogenetic mechanism, including congenital spinal dermal sinus tract (CSDST), limited dorsal myeloschisis (LDM), and their mixed lesions ${ }^{3,5,6,11,25,39,42)}$. A comprehensive study on these focal spinal nondisjunctional disorders (FSNDs) can be found in a recent book chapter titled "Limited dorsal spinal nondisjunctional disorders ${ }^{\Re(3)}$.

- Received : April 21, 2020 •Revised : July 29, 2020 •Accepted : July 29, 2020

- Address for reprints : Dachling Pang

Department of Paediatric Neurosurgery, Great Ormond Street Hospital for Children, NHS Trust, Great Ormond Street, London WC1N3JH, UK Tel : +44-20-7405-9200, Fax : +44-20-7813-8279, E-mail : pangtv@aol.com, ORCID : https://orcid.org/0000-0002-6603-6546 


\section{EMBRYOGENETIC MECHANISMS}

\section{The basis for grouping these conditions as one entity}

Primary neurulation can be broadly divided into four main stages, occurring sequentially at each axial level of the embryo, during that the neural plate goes through 1) formation, 2) shaping, 3) dorsal bending, and 4) closure of the neural groove to become the primary neural tube ${ }^{2)}$. At the tissue level, the last stage actually consists of a complex sequence of three overlapping events $^{2,14,31,41)}$ (Fig. 1) : 1) A broad overlapping surface epithelium (SE) and neuroepithelium (NE) junction of several cells' thickness is formed at the paired neural folds. It occurs as the neural folds progressively elevate and converge towards the dorsal midline, and the NE cells enlarge in height and drag the flattened SE cells onto their dorsal surface. 2) The two overlapping epithelia through progressive delamination become completely separate, known as disjunction.
The delamination process starts with an inter-epithelial space in the middle part of the interface of the two epithelia. As the space expands, it acquires a crescent shape because the basal lamina of the two epithelia remains attached at the ventro-lateral extreme of their contact (ventral contact point). While the two neural folds are progressively approaching each other to close the dorsal midline gap, the inter-epithelial space extends further dorsally towards the dorsal midline. Ventrally, the basal lamina bridge at the ventral contact point finally breaks down, and the two epithelia separate there. A new basal lamina then forms on the "inter-epithelial surfaces" of each of the two epithelia. When the inter-epithelial space on each side reaches the dorsal-most meeting point of the two epithelia and separates them, it consummates disjunction. 3) During the same period, fusion of the two epithelial layers at the dorsal midline occurs. Fusion is preceded by apposition of the two opposing sets of epithelia at the tips of the two neural folds, and formation of intercellular adhesion at contact points,

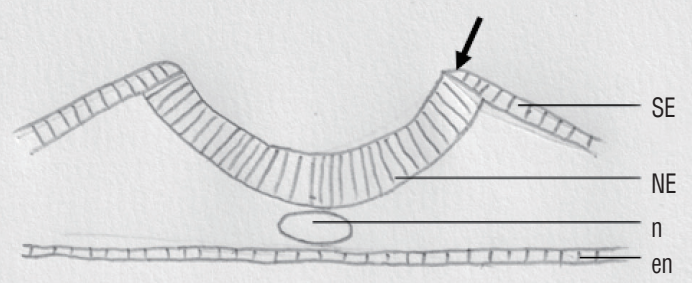

(A)

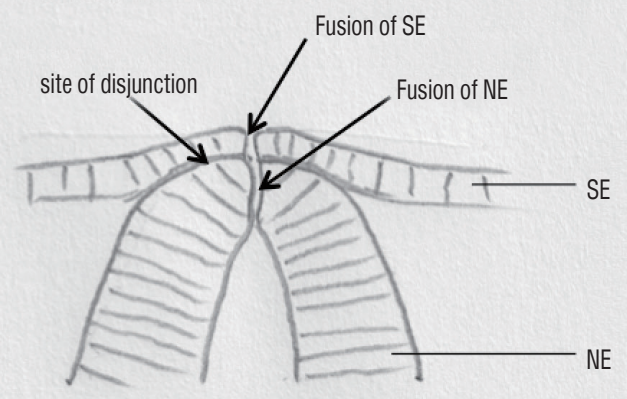

(C)

Fig. 1. Normal primary neurulation. Diagrams showing the major steps in closure of the neural groove in an axial level. A : Elevation of the neural folds (arrow). B : Progressive elevation of the neural folds. Delamination at the neuroepithelium - surface epithelium interface. C: Components involved in the final phase in closure of the neural groove. $D$ : Closed neural tube at an axial level. Reused from Wong et al. ${ }^{43)}$ with permission from Springer Nature. $\mathrm{SE}$ : surface epithelium, NE : neuroepithelium, $\mathrm{n}$ : notochord, en : endoderm. 
which are initially discontinuous from superficial to deep ${ }^{33}$. In normal embryos, completion of neural groove closure at an axial level is marked by the appearance of a continuous basal lamina under the SE across the dorsal midline, and a continuous sheath of basal lamina around the NE (primary neural tube) at that level ${ }^{15)}$. There are two subtle details of these intercalated processes that are important to our embryogenetic hypotheses : first, fusion of the two epithelial layers likely proceeds independently of each other ${ }^{41)}$. Secondly, although the exact timing of epithelial fusion and disjunction is unknown, intuitively, fusion of the epithelia must precede disjunction of the SE and NE.

Faults occurring at a focal point during these last phases of neural groove closure give rise to FSNDs, which are characterized by nondisjunction and incomplete fusion of a focal, or "limited", segment anywhere along the future spinal cord down to the S1 or S2 cord level ${ }^{9,18,19,27)}$. Depending on the aberrant behaviours of the various primordial cells involved in the neural groove closure, several anatomical phenotypes of the final malformation are seen. In addition, the matured features of the subtype malformations of FSND are determined by individual or combined errors of the primordial SE and NE cells, as well as the mesoderm and neural crest cells (Fig. $2)^{31,32,37)}$.

At the molecular genetic level, the mechanisms of primary neurulation have only been partially elucidated. The different stages of primary neurulation have different key molecular players $^{20)}$. In FSNDs, the underlying faulty molecular events are likely confined to those related to fusion of the epithelia and delamination/disjunction occurring at the dorsal midline. One group of regulator proteins that may be targets in the genesis of FSND are the Rho GTPases, including Racl and Cdc42. Both Racl and Cdc42 are involved in the regulation of cellular protrusions in SE during dorsal midline fusion ${ }^{20,28)}$. As for the molecular mechanisms of nondisjunction, there is even scarcer information; one possibly point of aberration may occur during the caspase-dependent apoptosis of the cells at the SE-NE border ${ }^{20,44)}$. There are two general points about defective molecular mechanisms in FSNDs : 1) to account for the "limited" extent of a FSND lesion, a causative molecular de-
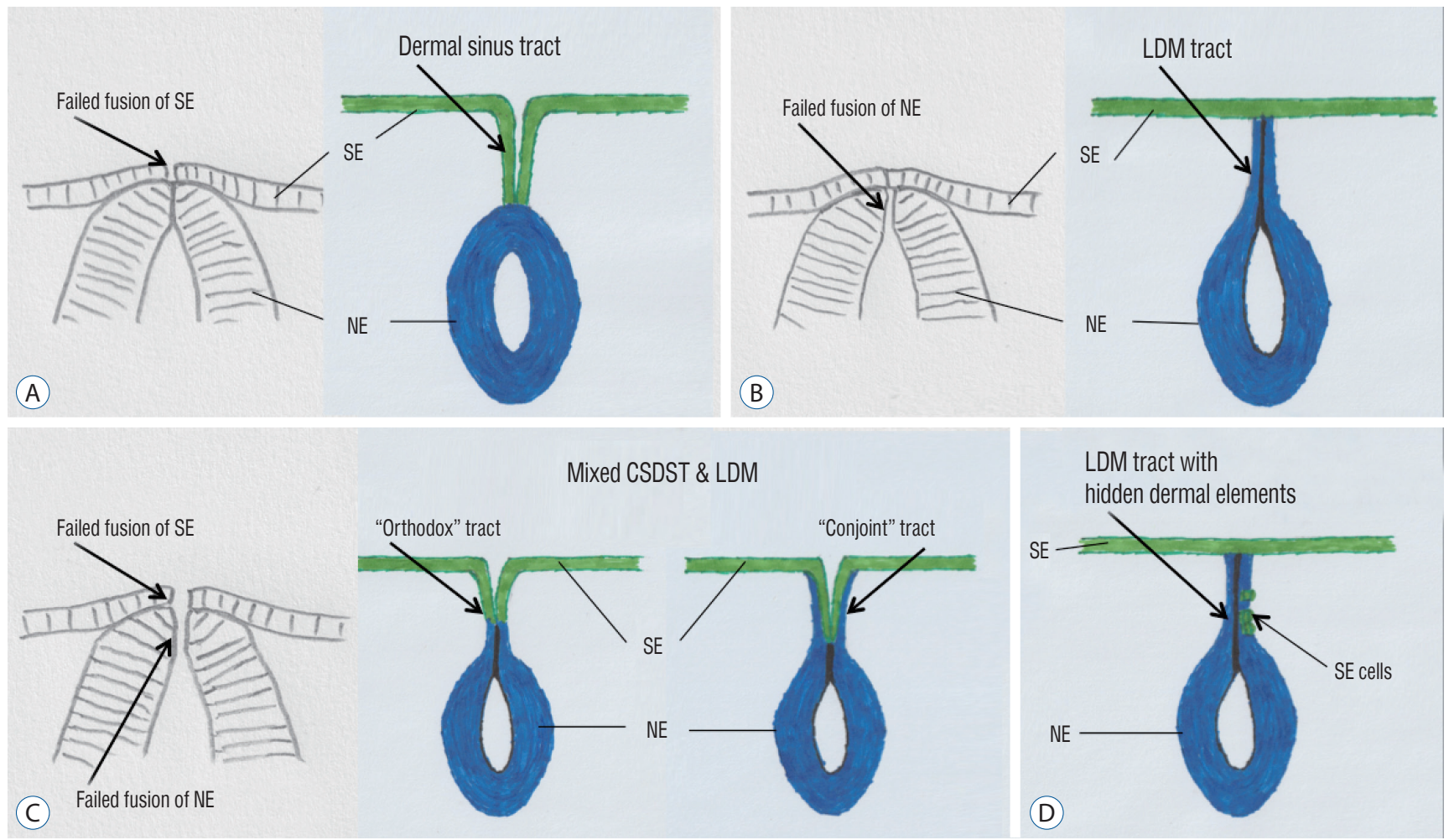

Fig. 2. Proposed embryogenetic mechanisms for different types of focal spinal non-disjunctional disorders. A : Congenital spinal dermal sinus tract (CSDST). B : Limited dorsal myeloschisis (LDM). C : Mixed CSDST and LDM - "orthodox" type and "conjoint" type. D : LDM with hidden dermal elements. SE : surface epithelium, NE : neuroepithelium. 
fect must involve a small clone of cells during neurulation; and the mechanisms concerned are probably cell-autonomous i.e., the presence of wild type cells nearby cannot correct the error within the affected cells ${ }^{20)}$. And 2) the different phenotypes described below may be due to different molecular faults. For example, Racl and Cdc42 only involve SE fusion; thus the clinical phenotypes due to their aberrations could be different from those affecting proteins regulating $\mathrm{NE} \mathrm{fu-}$ $\operatorname{sion}^{20,28)}$.

The embryogenetic mechanisms for the subtype FSND malformations, described in terms of morphological changes in the embryonic tissue, are as follows (Fig. 2) : 1) a CSDST develops, when fusion of the SE fails at a focal point, but the underlying NE fusion has at least been represented by some intercellular adhesions, and disjunction at this focal point does not occur. In this situation, closure of the primary neural tube immediately cranial and caudal to this focal nondisjunction spot is unhindered, but the gaping SE is persistently linked with the NE at this focal spot. This link, a midline gap in the converging SE and, below it, between the dorsal scleromyotomes in opposite sides of the embryo, remains very narrow. Further unimpeded development of the surrounding normal full-thickness dorsal myofascial tissues around the midline strip progressively sets the primary neural tube into its normal, primarily intraspinal location. However, a dorsomedian tract of SE tissue persists as the original link between the closed primary neural tube and the still slightly gaping epithelial surface. The tract is firmly anchored on the SE side because its component cells are still essentially part of the SE, but its deep-end attachment to the NE cells after closure of the neural groove may not be firm. The deep end of the tract could therefore be dislodged from the underlying neural tube, by cellular movements during normal development of the neural crest cells, meninges and scleromesoderm; so that the inner anchorage of the tract may end short of the spinal cord but on the meninges or even the musculofascial layers.

2) A LDM develops, when the fusion of the NE fails at a focal point but the overlying SE fusion has at least been represented by some intercellular adhesions, and disjunction at this focal point also does not occur. Like the reverse of the development of a CSDST, the SE gap is closed, but because NE fusion and disjunction never happen at the focal spot, the NE remains linked with the SE at the spot. This link, a midline gap in the converging NE and, above it, between the dorsal scleromyotomes in opposite sides of the embryo, remains very narrow. Further unimpeded development of the surrounding normal full-thickness dorsal myofascial tissues around the midline strip progressively also sets the primary neural tube into its normal, primarily intraspinal location.

However, a dorsomedian tract of NE tissue (vs. SE tissue in CSDST) persists as the original link between the focally gaping primary neural tube and the closed epithelial surface ${ }^{24)}$. The tract is attached on the undersurface of the SE, and affects the integration of mesodermal tissue at that focal area to form normal full-thickness skin.

3) A tract with mixed LDM and CSDST develops, when fusion of NE and SE both fail and disjunction never occurs, often in an "orthodox" manner with the inner portion of the tract containing NE tissue while the outer tract consisting mainly of SE. The "pulling forces" along the outer and inner portions of the tract during embryogenesis will determine the relative proportion of the two kinds of tissues in the final configuration. However, a "conjoint" type may also occur, where the entire tract is lined by both SE and NE elements. These mixed entities are rare but may easily elude detection ${ }^{5,11)}$.

4) As for LDM with hidden dermal element - LDMs with dermal elements but without a sinus tract, the origin of the SE cells could be from pluripotent cells near the dorsal midline, or from SE cells somehow being included during the formation of the LDM stalk ${ }^{5,25)}$.

5) FSND with spinal cord lipoma : dorsal spinal cord lipomas have been known to be associated with LDMs, either directly adjacent to the LDM stalk or continuous with it. Since both transitional and dorsal lipomas are thought to arise from premature disjunction, during the same embryogenetic period as nondisjunction; it is not surprising that nondisjunction and premature disjunction disorders may coexist ${ }^{25}$. Equivalent parallel faults may also account for the coexistence of CSDST and lipomas.

And 6) FSND with split cord malformation : the fibroneural stalk or dermal sinus tract found in some cases of split cord malformation may in fact be the remnant the dorsal portion of an anomalous ecto-endodermal fistula resulting from aberrant early gastrulation ${ }^{21,23)}$, which is the embryogenetic basis for split cord malformation. 


\section{PATHOLOGICAL ANATOMY AND CLINICAL MANIFESTATIONS OF FOCAL SPINAL NONDIS- JUNCTONAL DISORDERS}

Variations in the exact cellular types and histological configurations of FSND lesions account for the spectrum of nondisjunctional disorders, and partial atresia of a tract may explain certain variants of the full forms ${ }^{8)}$. Concerning the documentation of the "level" of a FSND, it should reflect the spinal cord level of the initial causative nondisjunctional error, and accordingly, it should be the level of the laminar defect through which the fibroneural stalk or sinus tract passes.

\section{Pure CSDST}

\section{Pathological anatomy}

A dorsal midline dermal sinus tract (a narrow tubular structure lined by squamous epithelium) is the essential feature of a CSDST ${ }^{13,40)}$. The size of the ostium in the skin of this tract is variable but usually small (Fig. 3). How deep the tract penetrates is also variable; over $60 \%$ end intradurally, and some are firmly attached to the spinal cord (Figs. 4-6) ${ }^{43)}$.

Regarding their location along the vertebral column, over $60 \%$ of CSDSTs are in the lumbosacral spine; the rest are distributed over the thoracic and cervical regions ${ }^{43)}$. The shape of
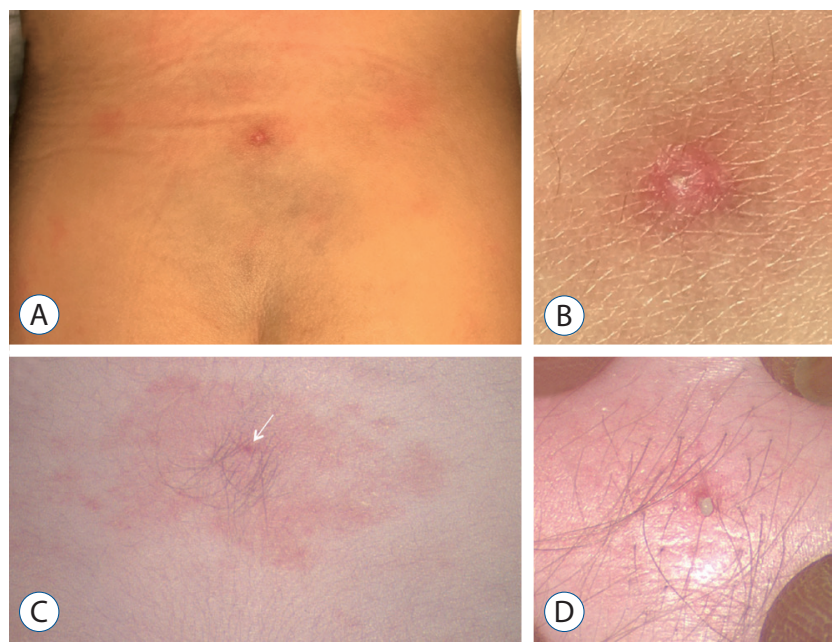

Fig. 3. A : Dermal sinus ostium, appeared as a pin-point area of dry scaling with surrounding red discoloration, but without soft tissue swelling. B : Magnified view of (A). C : Dermal sinus ostium, appeared as a dot of dark discoloration with surrounding hypertrichosis and pigmentation. Keratin material could be seen with light compression. D : Magnified view of (C). Reused from Wong et al. ${ }^{43)}$ with permission from Springer Nature. a sinus tract on the sagittal plane varies depending on its level of origin, because the spinal cord ascends along the vertebral column for a fair distance during development due to their discrepant growth rates. A lumbosacral CSDST typically takes on a $\mathrm{V}$-shape with the apex pointing at exactly the laminar level of its nondisjunctional error; its subcutaneous tract descends caudally to reach the lamina, and from thence it ascends towards the thecal sac. With more cranial lesions, the subcutaneous tract becomes progressively more horizontal until it points cranially towards the dura in cervico-thoracic lesions. At the skin level, other skin stigmata may sometimes accompany the sinus ostium (Fig. 3). At the laminar level, the tract may pass through the interspinous ligament, or through
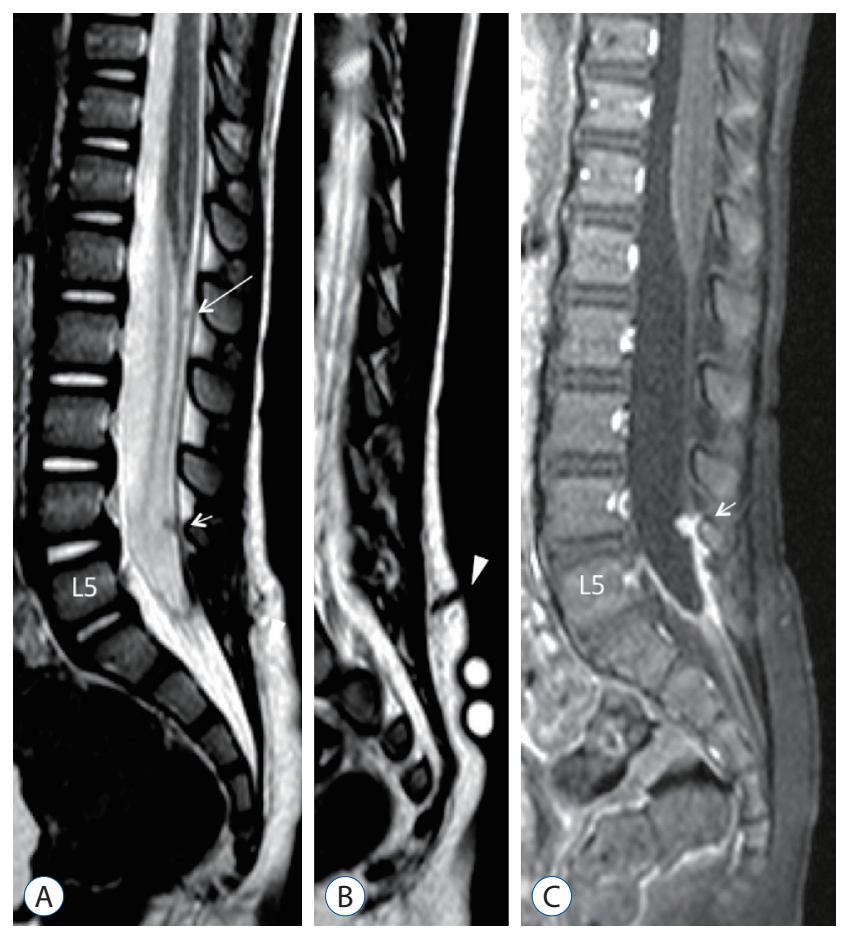

Fig. 4. MRI images of a 22-month-old with a dermal sinus tract, which as confirmed intraoperatively, has the skin ostium at $L 5$ spinous process level (Fig. $3 A$ and $B$ ), passes along the caudal aspect of $L 5$ laminae, and terminates on the dorsal surface of the conus. A : Mid-sagittal T2weighted MRI image showing a tiny $\mathrm{T} 2$ hypointense intradural nodule at L1/L2 vertebral level (long arrow), and another slightly larger one at L4 vertebral level (short arrow). The intradural dermal sinus tract is beyond the resolution power of MRI. B : Paramedian sagittal T2-weighted MRI image showing a dermal sinus tract from the skin ostium extending into the subcutaneous fat (arrowhead). C : T1-weighted MRI with gadolinium injection image, corresponding to $A$, showing that only the $L 4$ lesion (short arrow) and the end of the thecal sac become enhanced due to active inflammation. Reused from Wong et al. ${ }^{43}$ with permission from Springer Nature. MRI : magnetic resonance imaging. 

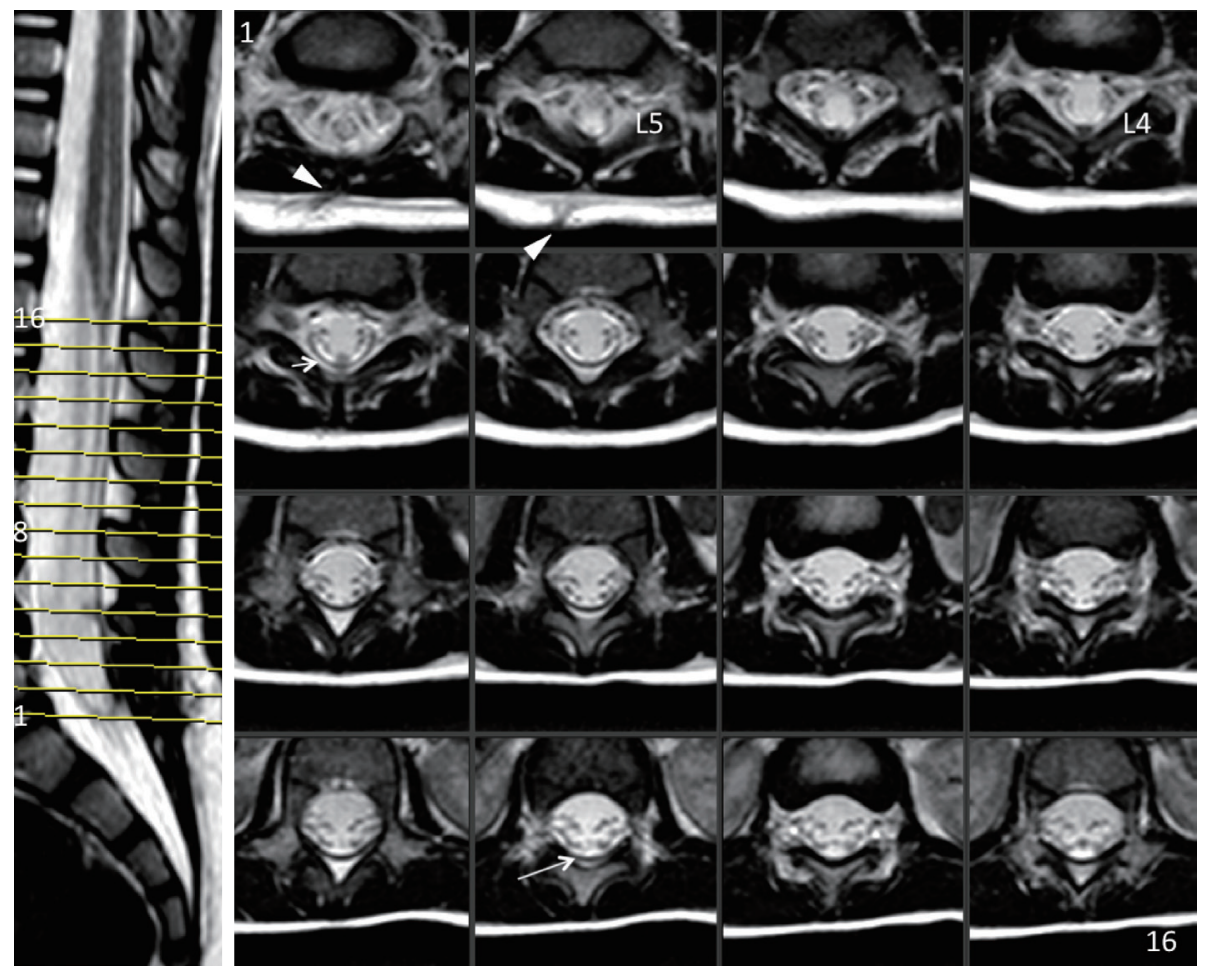

Fig. 5. Right panel: 16 serial T2-weight MRI axial cuts over the lumbosacral region of the patient shown in Fig. 4. Only the L4 nodule (short arrow), the skin ostium and subcutaneous tract (arrowheads), and vaguely the L1/L2 nodule (long arrow) are demonstrable by MRI. Left panel : T2-weighted MRI image with cut lines numbered 1 to 16 . Reused from Wong et al. ${ }^{43}$ with permission from Springer Nature. MRI : magnetic resonance imaging, L4 : left lamina of L4 vertebra, L5 : left lamina of L5 vertebra.
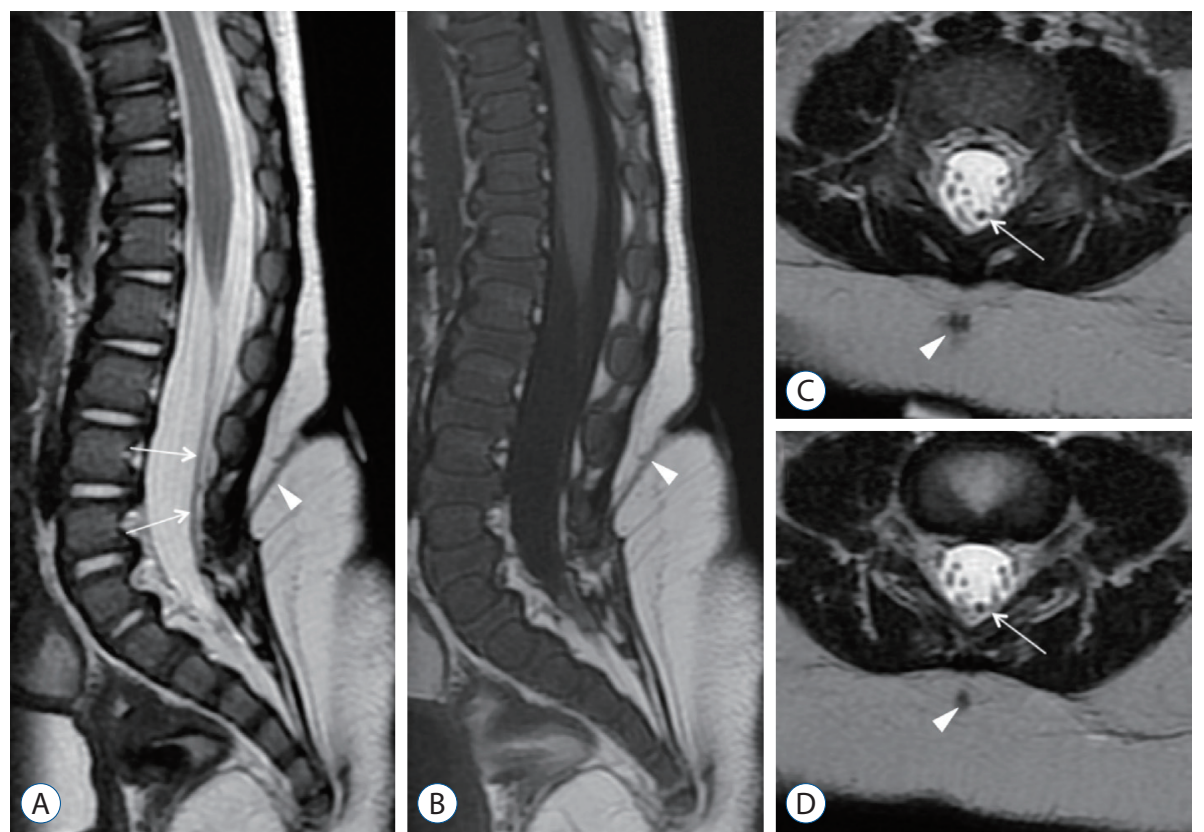

Fig. 6. MRI images of a 20-month-old with a dermal sinus tract. A, C, and D : T2-weighted MRI images. B : T1-weighted MRI image. Although there is marked abnormal signal at the skin level, the skin ostium is tiny (Fig. $3 C$ and D). There is a 2 vertebral levels difference between the skin ostium and where the tract located at the laminar level. The intradural tract (long arrows) appears as a structure that is slightly thicker and more T2 hypointense than normal nerve roots. Arrowheads indicated that the subcutaneous portion of the dermal sinus tract. Reused from Wong et al. ${ }^{43}$ with permission from Springer Nature. MRI : magnetic resonance imaging. 
a bifid spinous process or lamina. The tract then penetrates the dura but can also run between the dural layers for a short length before becoming intradural. Within the thecal sac, it may be adherent to the nerve roots or filum and, until proven otherwise, one should always assume all sinus tracts reach the spinal cord. Anywhere along the sinus tract, a dermoid cyst may form from existing keratin material, and it can even be intramedullary (Fig. 7). In a report of ten intramedullary spinal dermoid cysts, nine had a traceable $\operatorname{CSDST}^{7}$. The rare occurrence of spinal dermoid cyst without a sinus tract is probably due to isolated sequestration of pluripotent SE cells or atresia of the outer tract ${ }^{8}$.

Histologically, a dermal sinus tract is lined by keratinizing stratified squamous epithelium (Fig. 8A and B). Other components in variable abundance include hair follicles and shafts, mesenchymal derivatives such as blood vessels and fibrous tissue (Fig. 8A-C), and occasionally even nerve fibers. Keratin material fills the lumen of the tract and the cavity of dermoid cysts (Fig. 8D). Sometimes, the lumen of part of the tract may be obliterated (Fig. 8C). In slender tracts, a transitional zone of epithelial to non-epithelial tissues can be observed over the tract's deep end (Fig. 8E). Within the CSDST, inflamed granulation tissue containing mixed neutrophils, plasma cells, lymphocytes, and histiocytes is consistently found (Fig. 8A-C) ${ }^{4,13,38)}$. It is due to chemically induced inflammation from keratin accumulation, and may also be secondary to bacterial infections from sinus tract's communication with the skin surface.

\section{Clinical manifestations}

The age of presentation has a wide range; in most series, the mean is 3 years or below, with some patients first diagnosed in their 30's or even 50's ${ }^{43}$. The commonest presentation is skin stigmata, usually a cutaneous pit, frequently associated with pigmentation, haemangioma, skin tag, subcutaneous lipomas, or hypertrichosis (Fig. 3). Associated subcutaneous lipoma should arouse the suspicion for an associated spinal cord lipoma, and hypertrichosis for split cord malformation. In many published series, over $40 \%$ of patients had neurological deficits involving limbs and/or bowel and bladder. In infected cases and in patients harbouring large intradural epidermoid/ dermoid cysts, neurological deficits may arise catastrophical$1 y^{43)}$.

In actual practice, there is uncommonly a definitive history
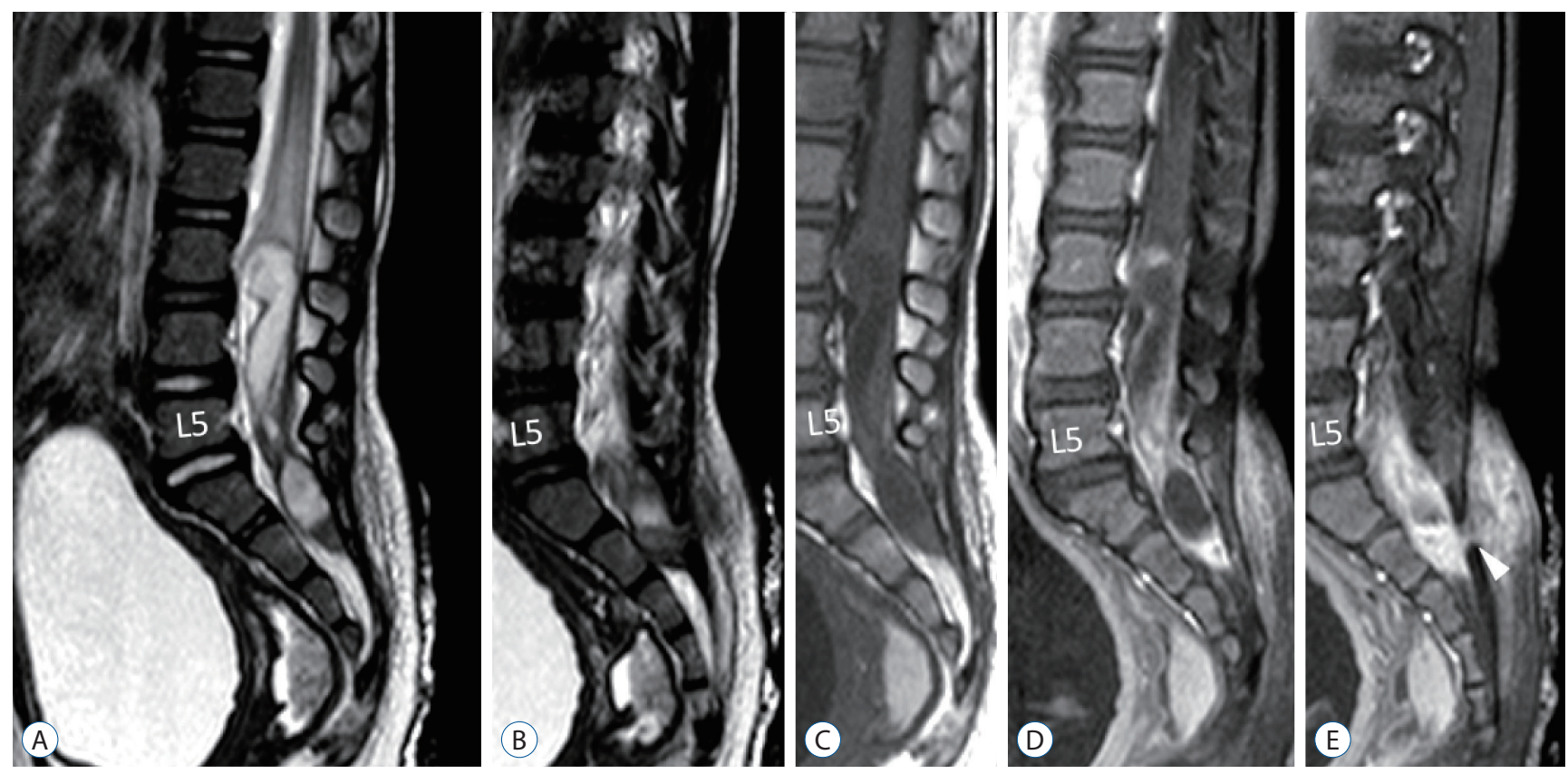

Fig. 7. MRI images of a 25-month-old with a large intradural dermoid cyst. A and B : T2-weighted images. C:T1-weighted image. D and E : T1-weighted with gadolinium injection. The dermoid cyst, spanning 5 vertebral levels, extends from L3 to S2. It is heterogenous in signal intensity, but the main bulk of it is T2-hyperintense, mildly T1-hypointense, and demonstrates periphery gadolinium enhancement. There is also marked gadolinium enhancement in the subcutaneous tissue signifying active inflammation. The intradural dermoid cyst communicates with an outside dermal sinus tract at the caudal aspect of the $\mathrm{S} 1$ laminae (white arrowhead in E). Reused from Wong et al. ${ }^{43)}$ with permission from Springer Nature. MRI : magnetic resonance imaging. 

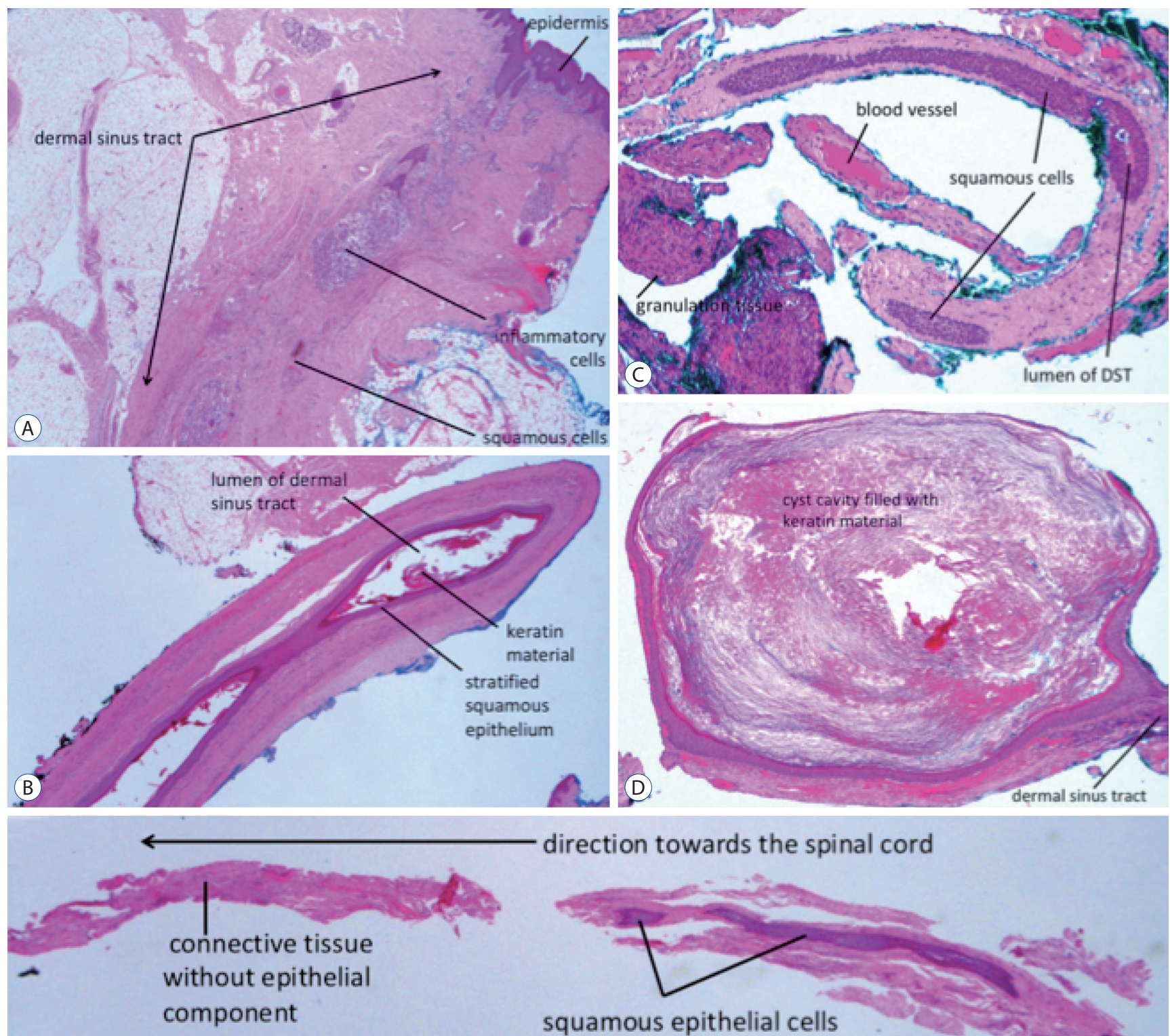

(E)

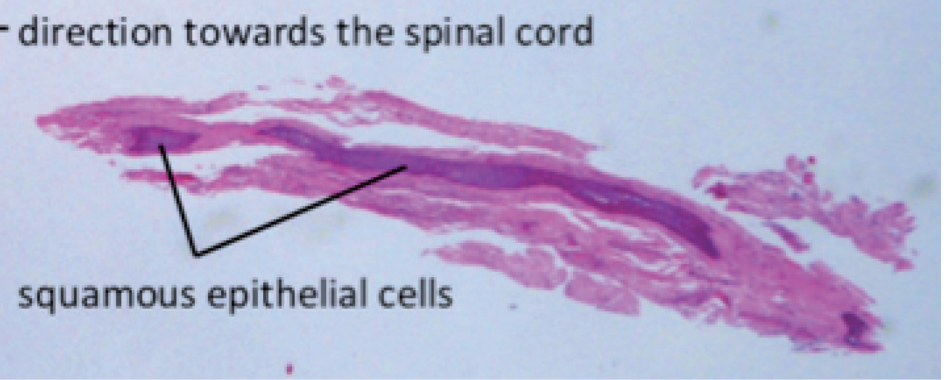

Fig. 8. Histological slides. A : Section through the most superficial portion of a dermal sinus tract. $B$ : The portion of the dermal sinus tract in the intraoperatively identified subcutaneous tissue. $C$ : The intradural portion of a dermal sinus tract (DST). A : The intradural portion of a DST. D : A dermoid cyst formed along a slender dermal sinus tract (the L1/L2 lesion in Fig. 4). E : The deepest end of a dermal sinus tract where a transition from epithelialized tissue to connective tissue totally devoid of it can be seen (haematoxylin and eosin stain). Reused from Wong et al. ${ }^{43}$ with permission from Springer Nature.

of discharge from a sinus ostium, which is seen only in 25\% of cases; and even rarer, inflamed skin around an obviously infected ostium or a deep-seated abscess, which occurs in less than $15 \%$. Paradoxically, in some series, a history of recurrent meningitis or active meningitis is found in up to $40 \%$ of cas$\mathrm{es}^{433}$.

\section{Pure LDM}

\section{Pathological anatomy}

The two constant features of all LDMs are 1) a cutaneous stigma and 2) an underlying fibroneural stalk anchoring the spinal cord to the skin lesion (Fig. 9). The cutaneous marker, a pearly crater of abnormal skin, commonly known as a "ciga- 


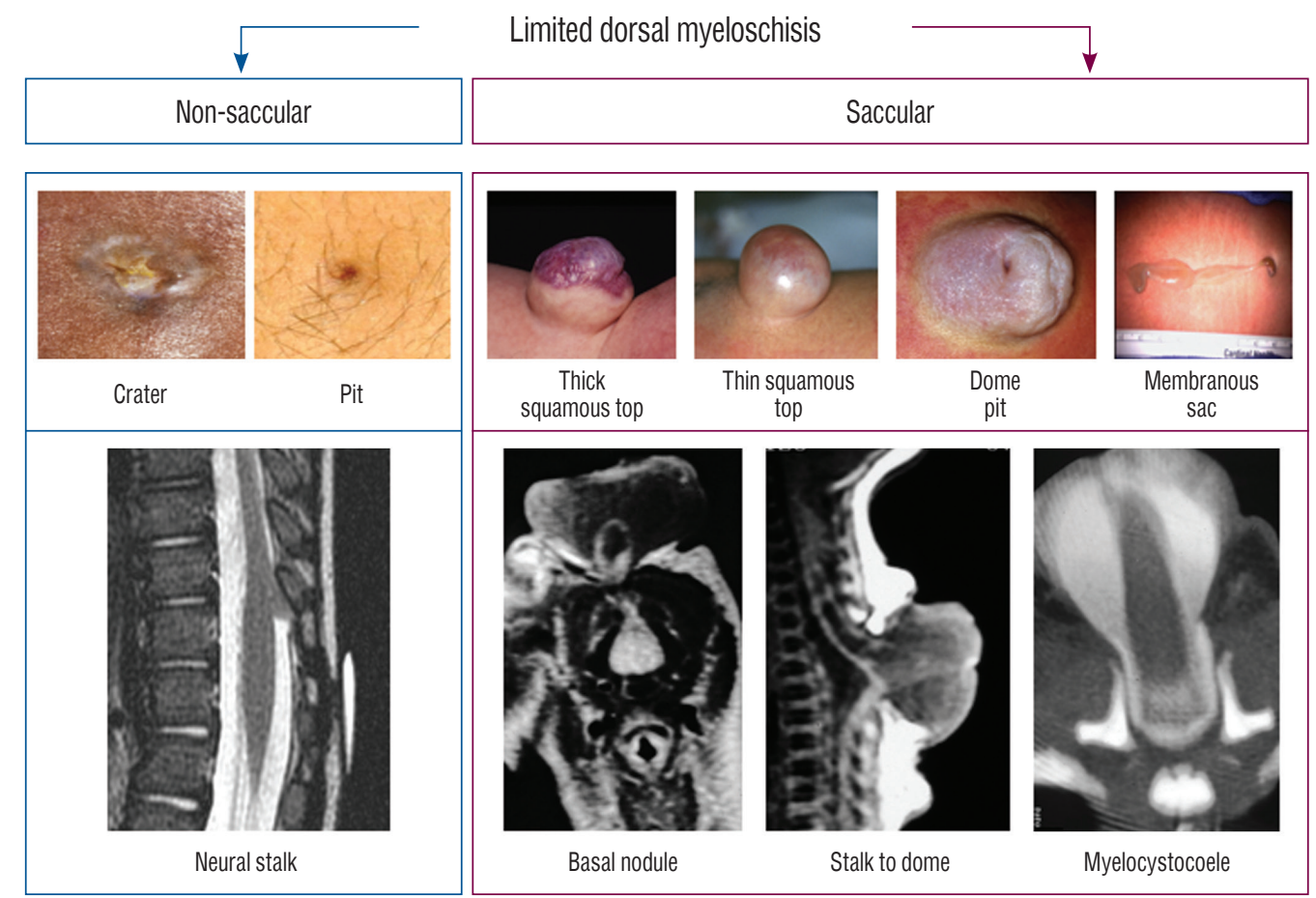

Fig. 9. Classification of limited dorsal myeloschisis (LDM) into non-saccular and saccular types, according to the 2 universal features of LDMs, one external, one internal. The top images depict the various skin signatures of either crater, pit, or the subtypes of sacs. The bottom images feature the internal fibroneural connections between the skin lesion and the spinal cord. Reused from Pang et al. ${ }^{25}$ with permission from Springer Nature.

rette-burn scar/mark", is due to hindrance on normal skin development by the stalk attaching to the undersurface of the SE (Fig. 2). In all instances, the fibroneural stalk, extending from the deeper side of the abnormal skin, ultimately merges with the spinal cord. Only one example of a discontinuous stalk has been documented ${ }^{8}$. In all LDMs, the spinal cord is tethered to the surface myofascial tissue by the fibroneural stalk $^{22,34,35)}$ and by the meningeal and other mesenchymal investments condensed around the stalk. The merge point of the stalk with spinal cord is always above the conus, indicative of this being due to faulty primary neurulation ${ }^{25}$. If there are lesions truly arising from secondary neurulation defects, but mimicking the morphology of a LDM, they should be classified as a different entity ${ }^{9,27)}$.

LDMs can be categorized by the external appearance of their skin lesion into flat (non-saccular) or saccular (Fig. 9). The flat LDM is recognisable either by a simple pin-point pit or a wider crater with "non-skin" squamous epithelium. Internally, the fibroneural stalk passes through the deep fascia, a bifid lamina/ the interspinous ligament, and the dura (Fig. 10). The intradural stalk of a lumbosacral lesion is seen, on mag-

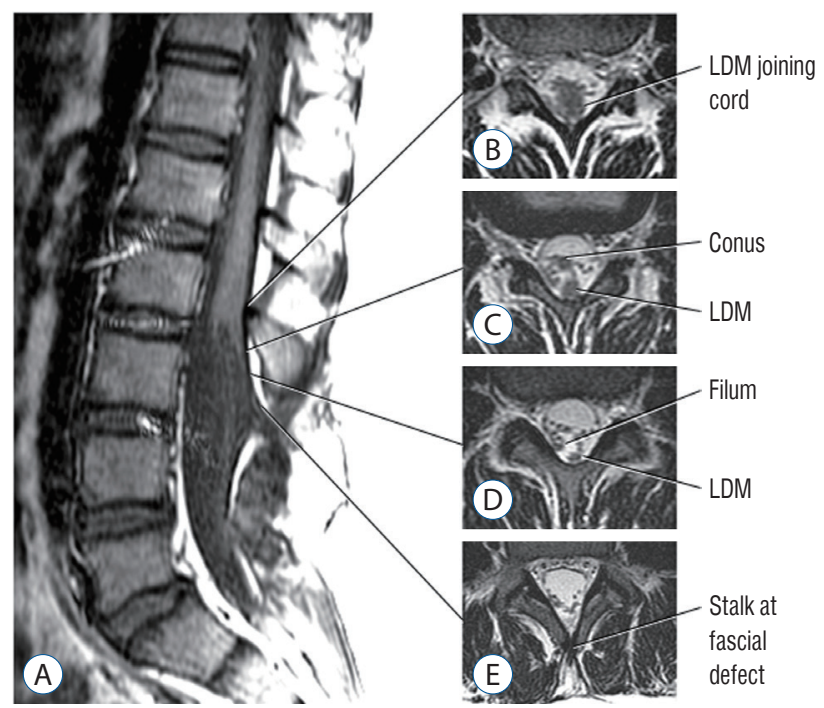

Fig. 10. Lumbar non-saccular (flat) limited dorsal myeloschisis (LDM). A : Sagittal MR showing subcutaneous fibroneural stalk going through laminar defect opposite $L_{3 / 4}$ entering dura opposite $L_{3 \prime}$ and joining spinal cord at $L_{2}$. B : Axial image where LDM stalk joins spinal cord. Note trapezoid shape of the cord-stalk junction. C : Intradural LDM stalk dorsal to the conus (low-lying). D : Intradural LDM stalk dorsal to thickened filum. E : Extradural LDM stalk at laminar defect. Reused from Pang et al. ${ }^{25)}$ with permission from Springer Nature. 
netic resonance imaging (MRI), as a separate longitudinal structure dorsal to the filum and, more rostrally, dorsal to the conus (Fig. 10A); at the stalk-cord union (merge point), it a trapezoid shape on axial MRI (Fig. 10B). The fibroneural stalk is $\mathrm{V}$-shaped on the sagittal plane, similar to the observation in CSDST, when it is in the lumbosacral region (Fig. 11); but it
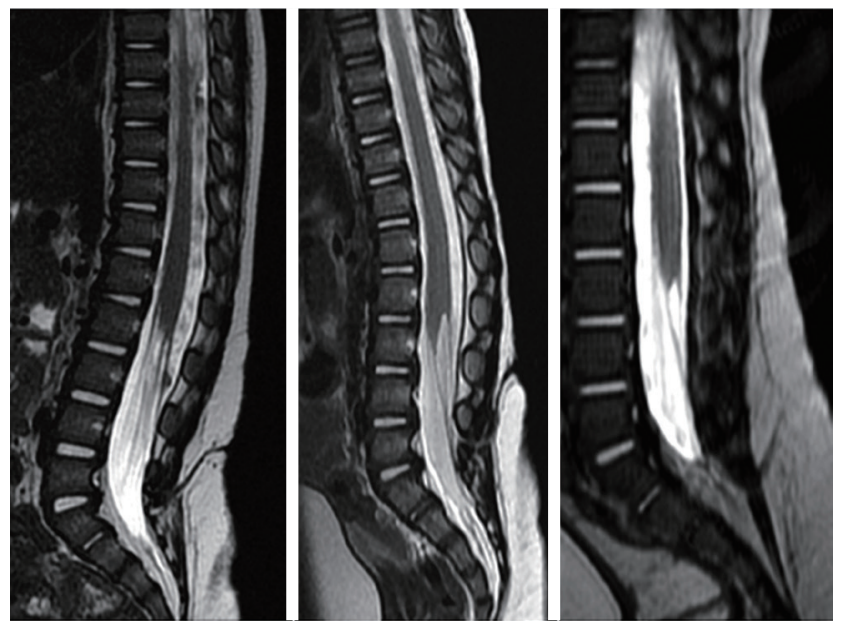

Fig. 11. Three cases of lumbar flat limited dorsal myeloschisis (LDM) with $\mathrm{V}$-shaped course of the LDM stalks. Left : Crater is at $\mathrm{L}_{4 / 5}$, stalk enters thecal sac at $S_{1}$, and joins spinal cord at upper margin of $L_{2}$. Middle : Crater at $\mathrm{L}_{3 / 4}$, stalk enters dura probably at $\mathrm{L}_{5} / \mathrm{S}_{1}$, and joins spinal cord at $\mathrm{L}_{2}$. Right : Crater at $\mathrm{L}_{4 / 5}$, stalk enters dura around $\mathrm{L}_{5} / \mathrm{S}_{1}$, and joins spinal cord at $L_{3}$. Reused from Pang et al. ${ }^{25)}$ with permission from Springer Nature.

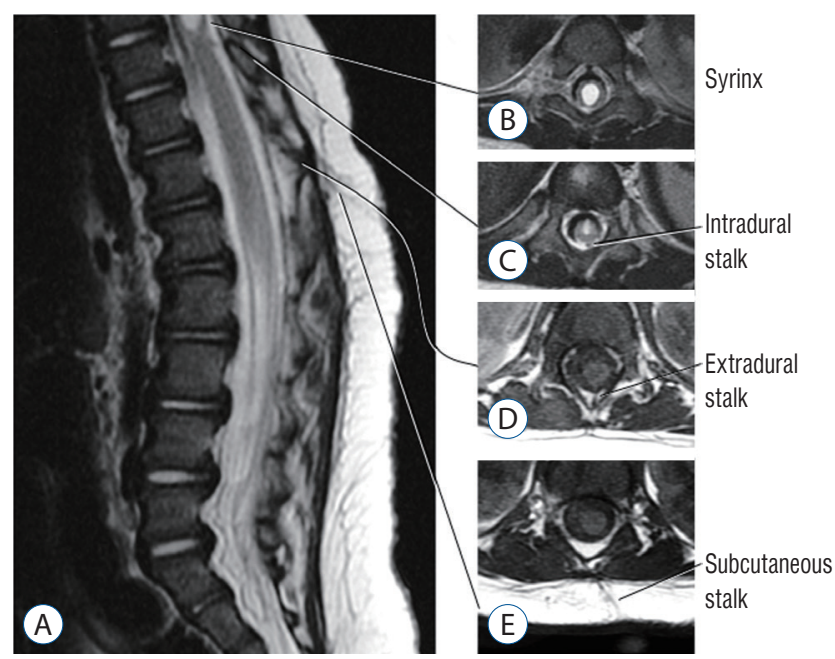

Fig. 12. Upward coursing limited dorsal myeloschisis (LDM) stalk in a thoracic flat LDM. A : Sagittal image shows skin crater at $L_{1}$, extradural stalk at $T_{12}$, dural entrance of the stalk above $T_{12}$, and joining of stalk to spinal cord at $T_{10}$. B-E : show axial image of the LDM stalk at corresponding points shown in (A). Reused from Pang et al. ${ }^{25)}$ with permission from Springer Nature. becomes progressively horizontal or slanting upwards when located more cranially (Fig. 12). The tethering effect to the cord can be very obvious when the fibroneural stalks are stout (Fig. 13), or when the cord is tented dorsally at the stalk-cord merge point (Fig. 14). In rare cases, the stalk appears to pull the cord archly dorsally towards the skin lesion to the extent that the cord seems to have deformed the overlying bone and myofascial layers (Fig. 15). In all these examples, the apparent dynamism of the tethering is obvious.

The saccular LDM arises with the initial embryogenetic architecture of a flat LDM, but the increasing hydrodynamic pressure of cerebrospinal fluid (CSF) soon changes its anatomical configuration drastically. CSF may be forced up along the slender dural sleeve surrounding the stalk, or through the
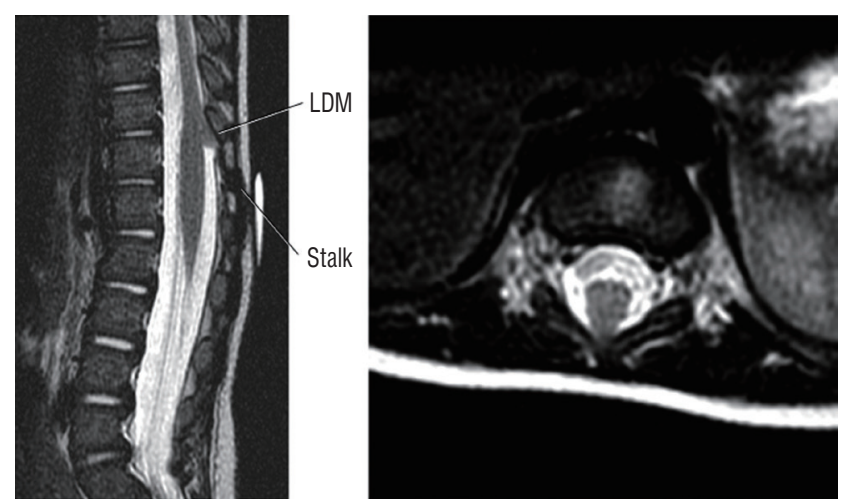

Fig. 13. Thick upward slanting fibroneural stalk in a thoracolumbar $\left(T_{11} /\right.$ $\mathrm{T}_{12}$ ) LDM. Reused from Pang et al. ${ }^{25)}$ with permission from Springer Nature. LDM : limited dorsal myeloschisis.
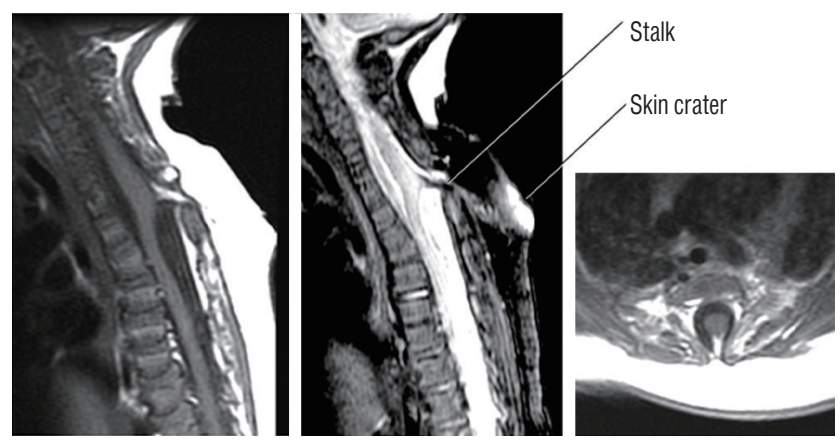

Fig. 14. Upper thoracic crater type limited dorsal myeloschisis (LDM) showing dorsal tenting of both the dural sac and spinal cord at the site of the stalk-cord junction, giving the appearance of taut tethering of the cord. Left : $T_{1}$ sagittal MRI. Middle : $\mathrm{T}_{2}$ sagittal MRI. Right : Axial MRI. Note skin crater, subcutaneous tract and intradural course of the stalk are well shown on the $\mathrm{T}_{2}$ sagittal image. Also, fat is seen within the stalk. Reused from Pang et al. ${ }^{25)}$ with permission from Springer Nature. MRI : magnetic resonance imaging. 
potential space within the fibroneural stalk's core to reach and distend the overlying skin into a fluctuant sac. Internally, the "sac" of saccular LDMs can have 3 types of content. First, the
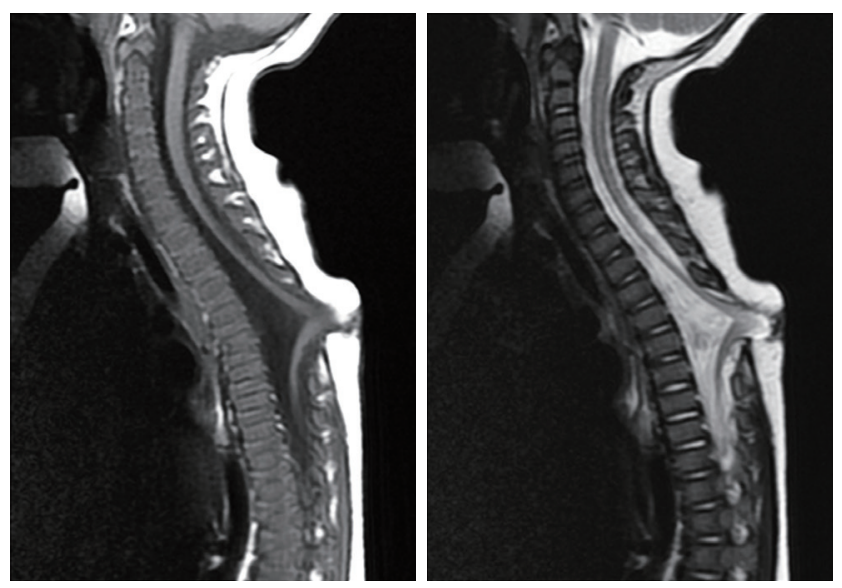

Fig. 15. $T_{5}$ LDM with non-saccular skin crater showing extreme dural displacement and kinking of the thoracic cord presumably due to "pull" by a short, stout fibroneural stalk. Left : $T_{1}$ sagittal MRI. Right : $T_{2}$ sagittal MRI. This child had early neurological deficits. Reused from Pang et al. ${ }^{25)}$ with permission from Springer Nature. MRI : magnetic resonance imaging. content is a segmental myelocystocoele : when the portion of the cord bearing the dorsal myeloschisis has an associated hydromyelia, the central lumen of the fibroneural stalk may be

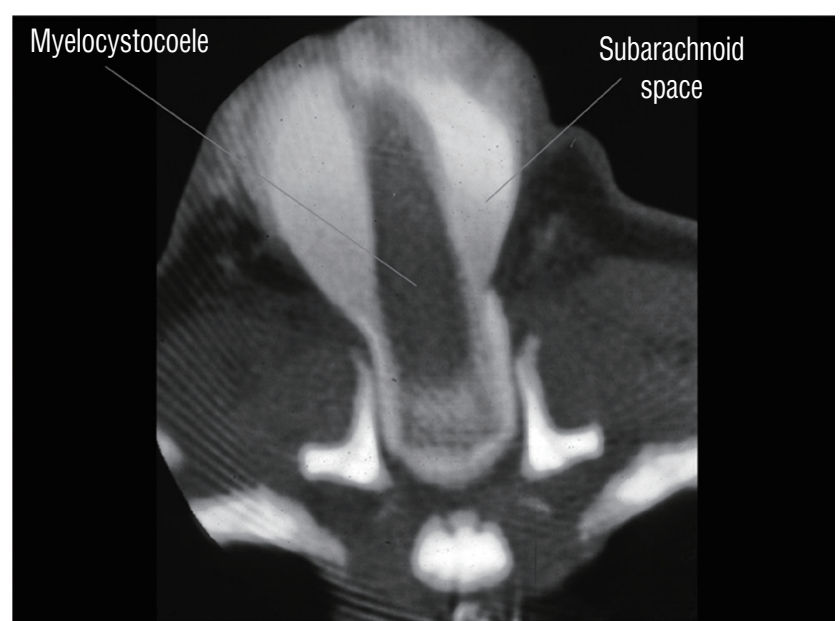

Fig. 17. Computed tomography myelogram of a cervical saccular limited dorsal myeloschisis with segmental myelocystocoele. The myelocystocoele sac does not contain contrast material, which remains in the subarachnoid space. Reused from Pang et al. ${ }^{25)}$ with permission from Springer Nature.

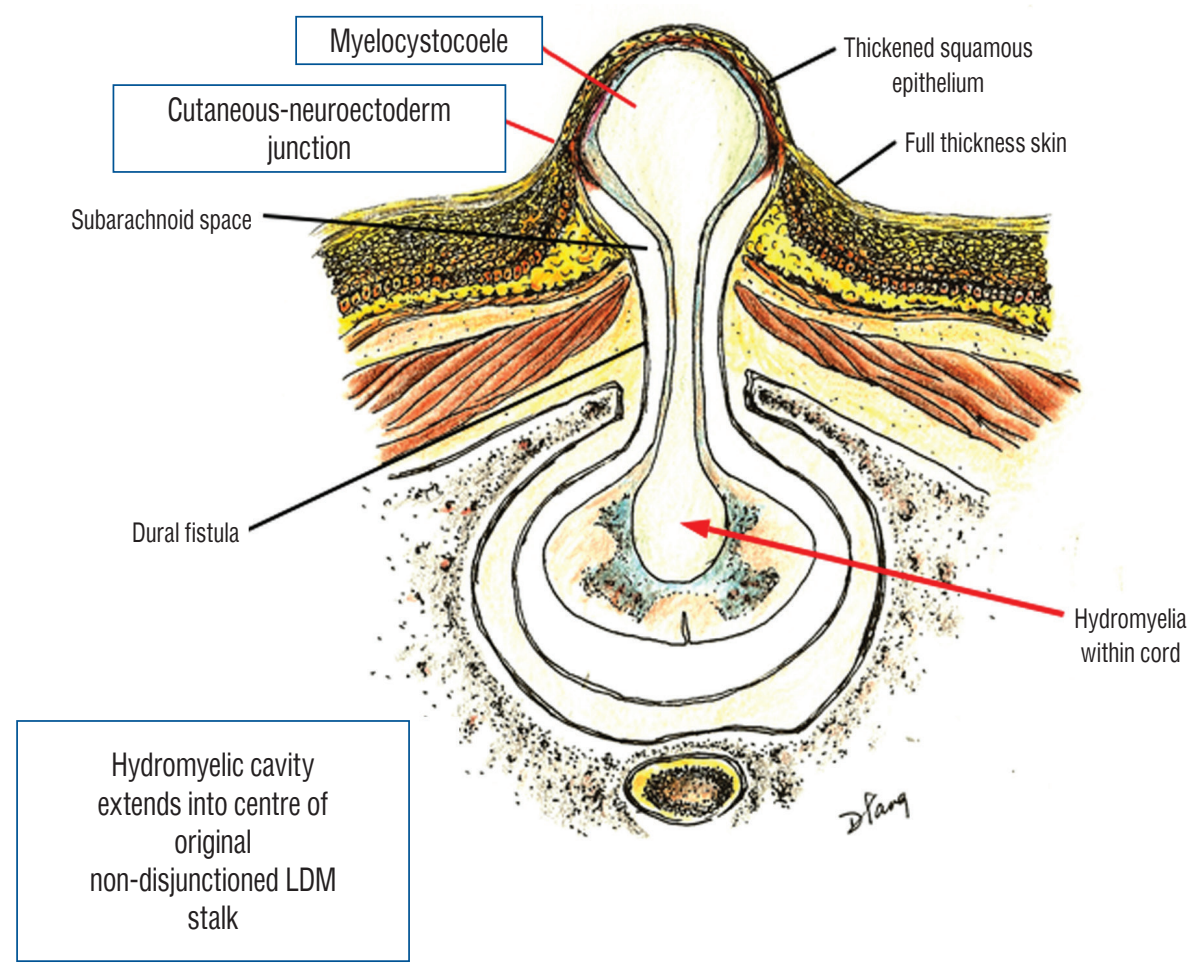

Fig. 16. Formation of saccular limited dorsal myeloschisis (LDM) with segmental myelocystocoele. Fluid from hydromyelic cavity in the underlying spinal cord dissects through the potential tubular space within the original cutaneo neuroectodermal tract and subsequently balloons out into an ependyma-lined myelocystocoele, a sac within an outer sac of distended subarachnoid cerebrospinal fluid. The sac is covered by a full-thickness skin base and a thickened, distinctly different squamous epithelial dome. Reused from Pang et al. ${ }^{25)}$ with permission from Springer Nature. 

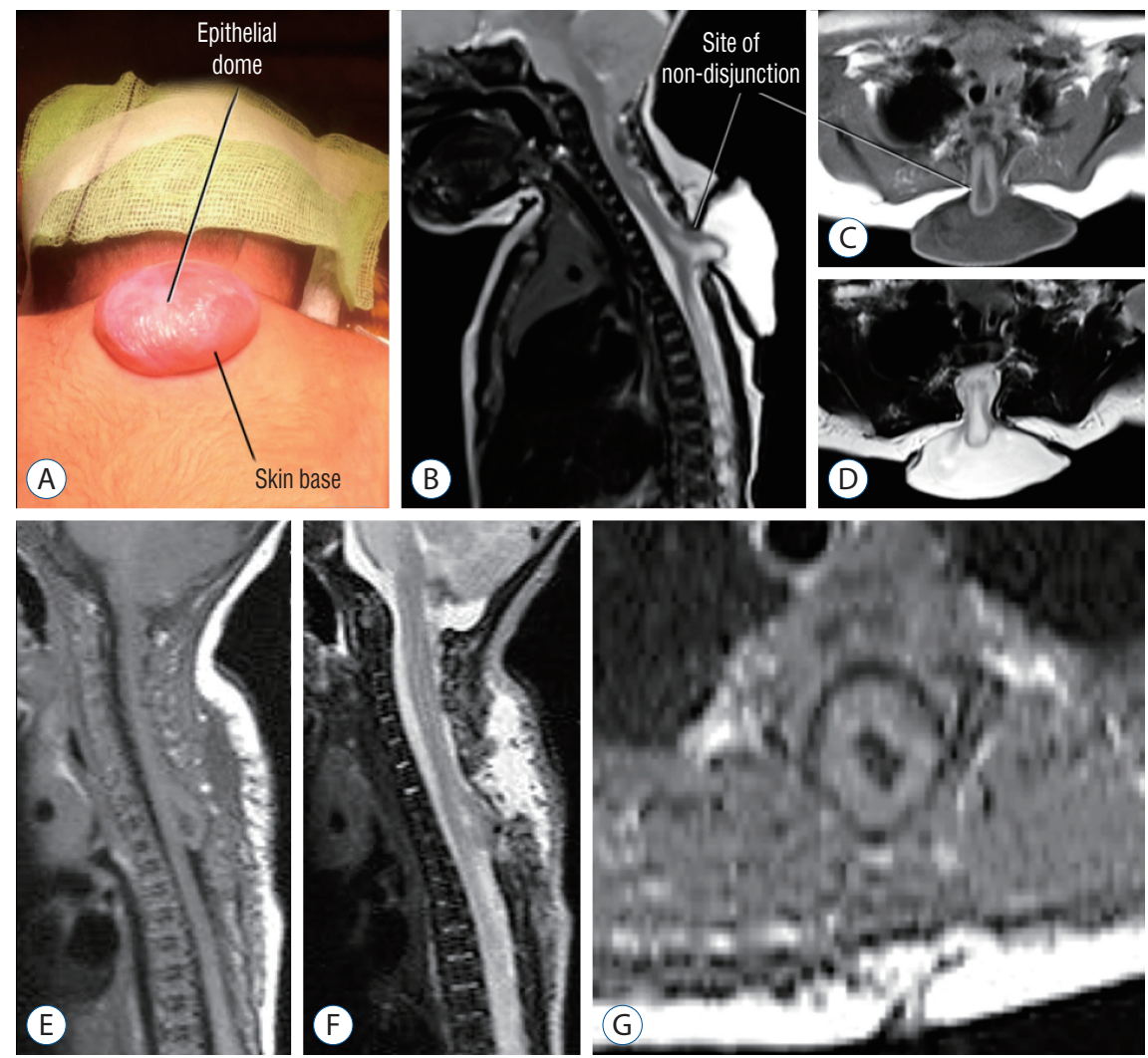

Fig. 18. High thoracic saccular limited dorsal myeloschisis with segmental myelocystocoele. A-D : Pre-operative. A : Photo showing the sac with the epithelial dome. B : Sagittal T2-weighted MRI. C : Axial T1-weighted MRI. D : Axial T2-weighted MRI. E-G : Postoperative MRI images. E : Sagittal T1weighted MRI. F : Sagittal T2-weighted MRI. G : Axial T1-weighted MRI. MRI : magnetic resonance imaging.
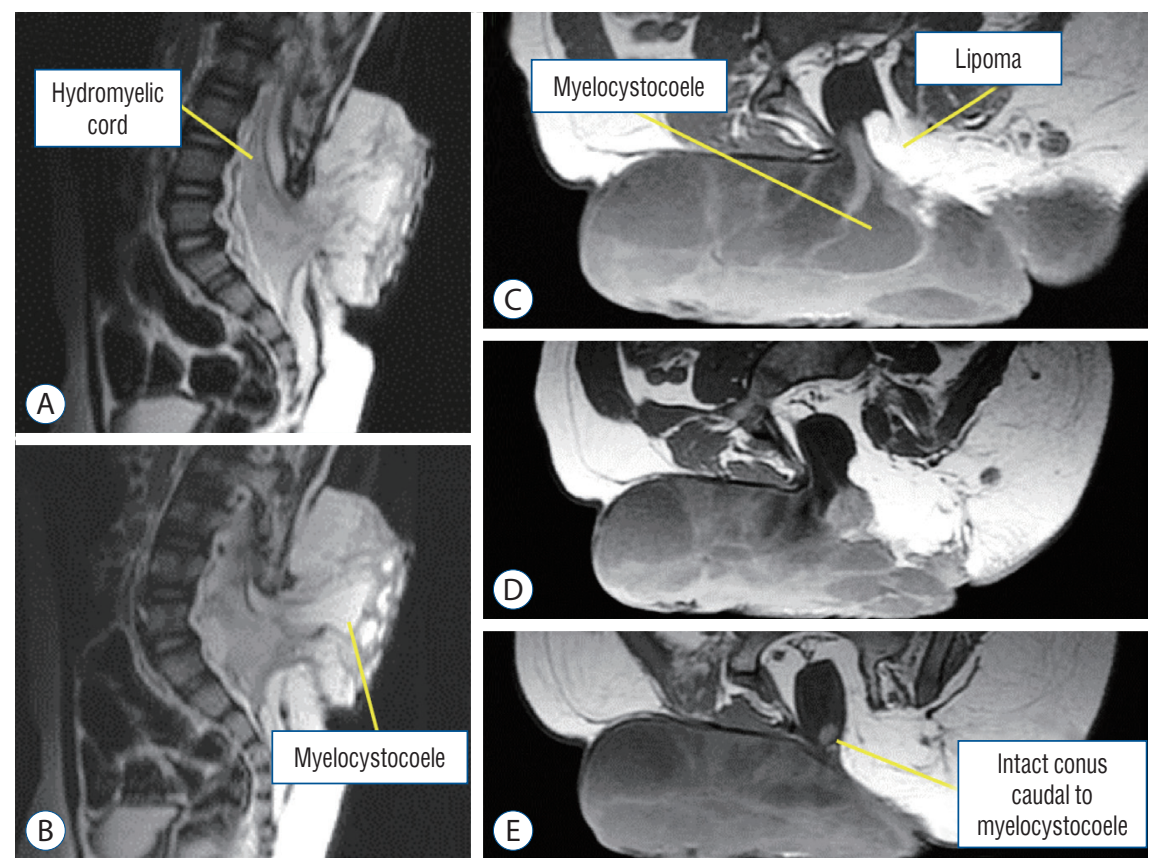

Fig. 19. Lumbar saccular limited dorsal myeloschisis with segmental myelocystocoele and lipoma in a 2.5 -year-old boy. A and B : Sagittal T2-weighted MRI. C-E : Axial T1-weighted MRI. MRI : magnetic resonance imaging. 


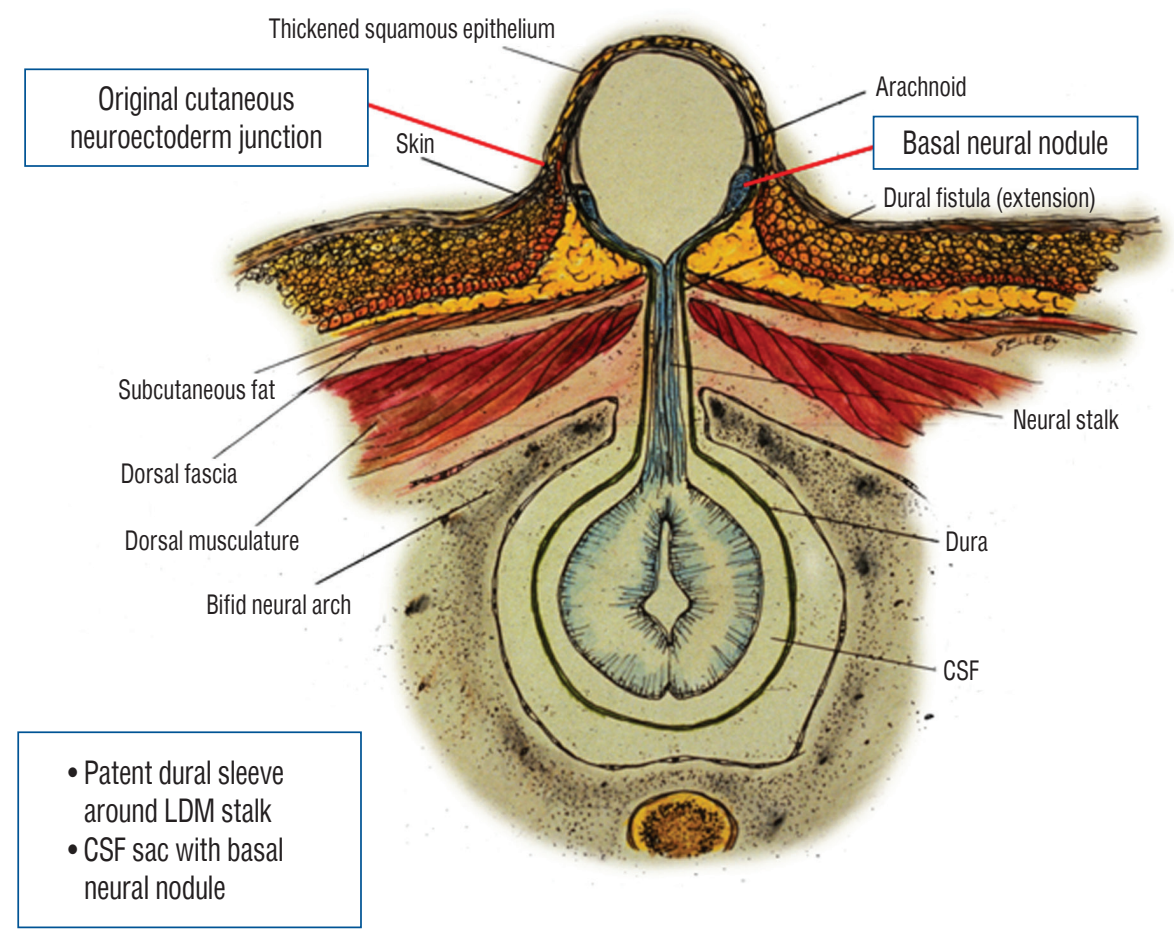

Fig. 20. Formation of a saccular limited dorsal myeloschisis (LDM) with basal neural nodule. In these cases, cerebrospinal fluid (CSF) dissects along the dural fistula ensheathing the fibroneural stalk and balloons out the less well supported midline epithelial layer to give a CSF-filled sac, whose base is skin-covered. The neuroectoderm at the original site of non-disjunction swells to become the basal neural nodule. Reused from Pang et al. ${ }^{25)}$ with permission from Springer Nature.

distended by CSF into a large myelocystocoele housed in an epithelium-covered sac (Fig. 16). This type is most commonly found in the cervical region (Figs. 17-19) ${ }^{29,34-36}$. Second, it has a basal neural nodule. When there is no hydromyelia, the fibroneural stalk and its central lumen remain compressed and narrow in its deeper course, but the superficial portion of the stalk swells into a fluid sac with basal neural nodules at its base, while retaining the original nondisjunctional attach-

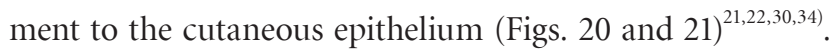
Third, it has a slender fibroneural stalk traversing the CSF sac to reach its dome (stalk-to-dome) : least commonly in saccular LDMs without hydromyelia, the normal meninges around the neural tube extends to ensheath the fibroneural stalk, and projects to reach the SE. CSF squeezes into the dural fistula containing the fibroneural stalk and ultimately distends the thinner, less well-supported squamous epithelial membrane on the surface into a CSF-filled, skin-based but epitheliumcapped sac. Strands of the fibroneural stalk traverse the fluid cavity of the sac to reach the part of the dome bearing the edge of the crater, where the nondisjunction occurred (Fig. 22).

Depending on the fluid pressure and the thickness of the

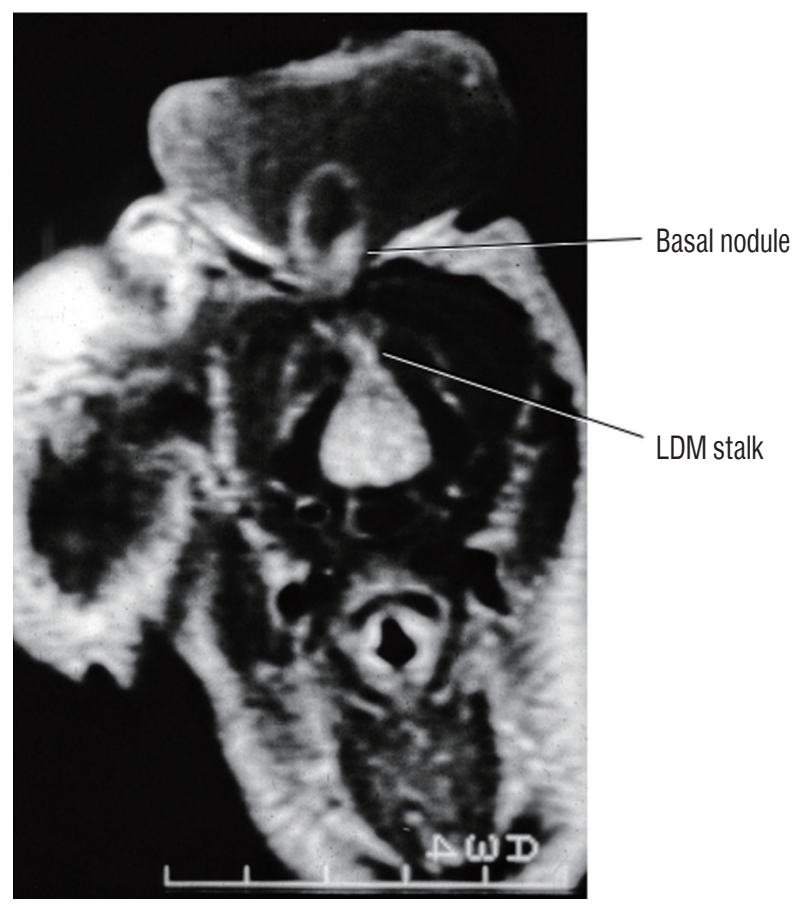

Fig. 21. Cervical saccular limited dorsal myeloschisis (LDM) with basal neural nodule within the base of the cerebrospinal fluid sac at the original non-disjunction site between cutaneous and neural ectoderms. Reused from Pang et al. ${ }^{25)}$ with permission from Springer Nature. 
apical epithelium and adjacent skin, the sac wall varies from the coarse, purplish, corrugated cap in the not-so-turgid tubular structures in many cervical saccular lesions, to the translucent membrane topping a tense lumbar sac, and finally to the giant, diaphanous bubble. A transitional form between

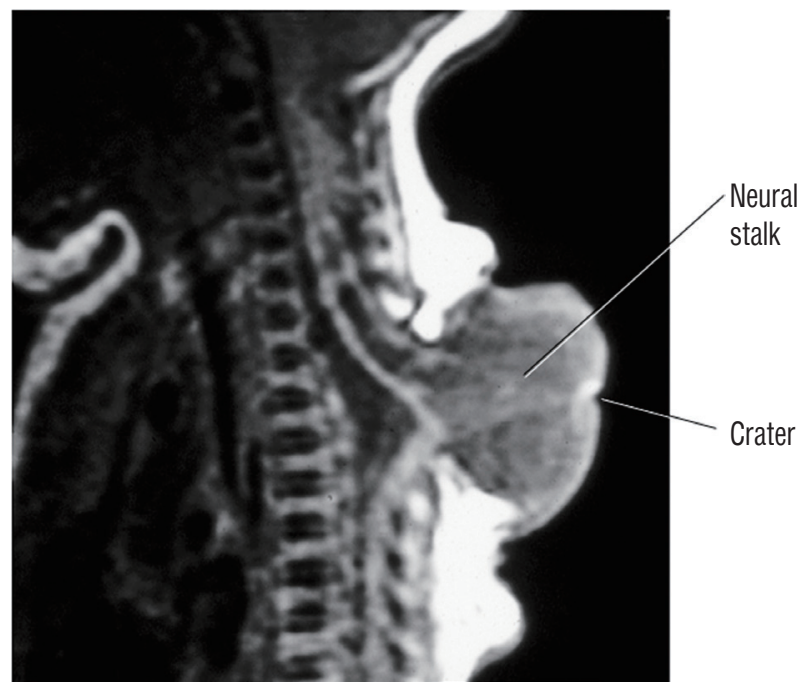

Fig. 22. Thoracic saccular limited dorsal myeloschisis with neural stalk that traverses the cerebrospinal fluid sac and reaches the small skin crater at the top of the cystic dome, presumably the original site of disjunction failure. Reused from Pang et al. ${ }^{25)}$ with permission from Springer Nature. saccular and flat LDMs can be observed, due to transient increase in fluid pressure during straining, in flat LDMs with the intermittently ballooning central crater (Fig. 23).

As with CSDST, registration of the spinal level of LDMs has been far from ideal. The challenge is due to the great difference in the level of the skin lesion, the level where the fibro-

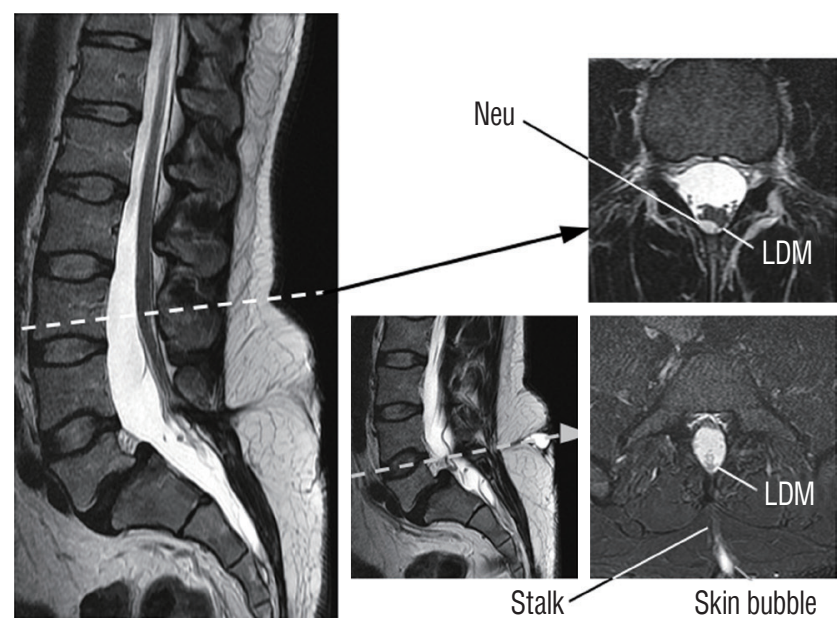

Fig. 23. Lumbar limited dorsal myeloschisis (LDM) showing a cerebrospinal fluid-filled "bubble" topped by squamous epithelium that distends only on straining. Note site of cord-stalk union is with slight dorsal "hump" on the cord outline, and a neurenteric cyst (Neu) right at this site. Reused from Pang et al. ${ }^{25)}$ with permission from Springer Nature.

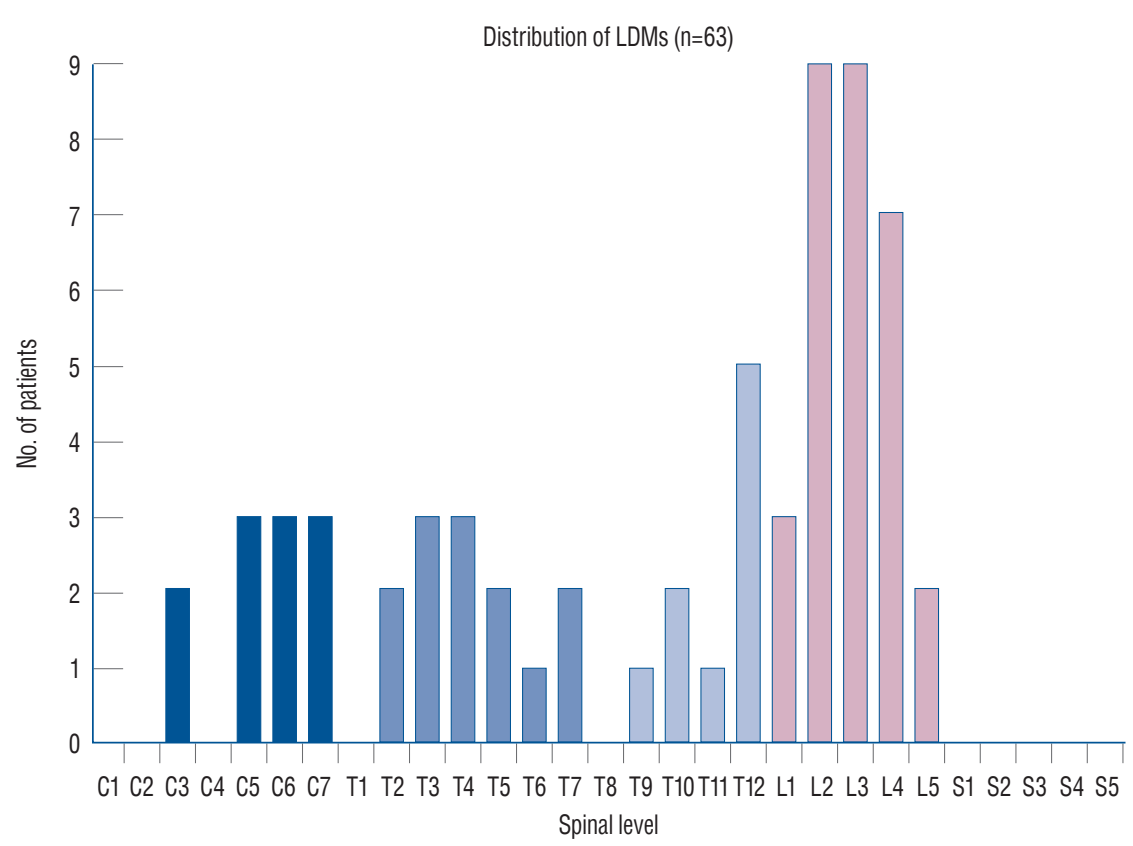

Fig. 24. Distribution of LDMs along the spinal axis. Designation of location is determined by the vertebral level where the fibroneural stalk attaches to the spinal cord. Note the two peaks at $L_{2}-L_{4}$ and $C_{5}-C_{7}$, and the absence of sacral lesion. Reused from Pang et al. ${ }^{25)}$ with permission from Springer Nature. LDM : limited dorsal myeloschisis. 


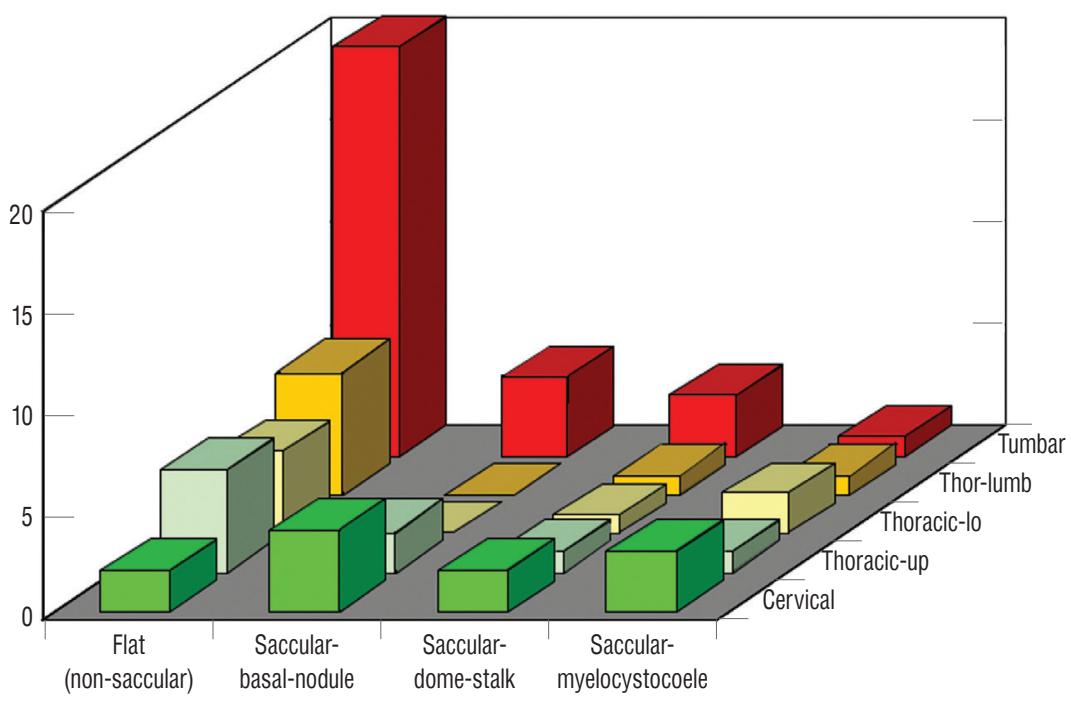

Fig. 25. Distribution of the four types of LDM classified according to external and internal features and assorted by regions of the spinal axis. The four types are flat (non-saccular), saccular with basal neural nodule, saccular with neural stalk reaching the cyst dome, and saccular with segmental myelocystocoele. Note preponderance of the flat LDM in the lumbar and lower thoracic regions. Saccular types are seen in both cervical and lumbar segments. The regions of the vertebral column are coded as : cervical (C1-C7); thoracic-up (T1-T5); thoracic-lo (T5-T11); thor-lumb (T12-L1); lumbar (L1L5). Reused from Pang et al. ${ }^{25)}$ with permission from Springer Nature.
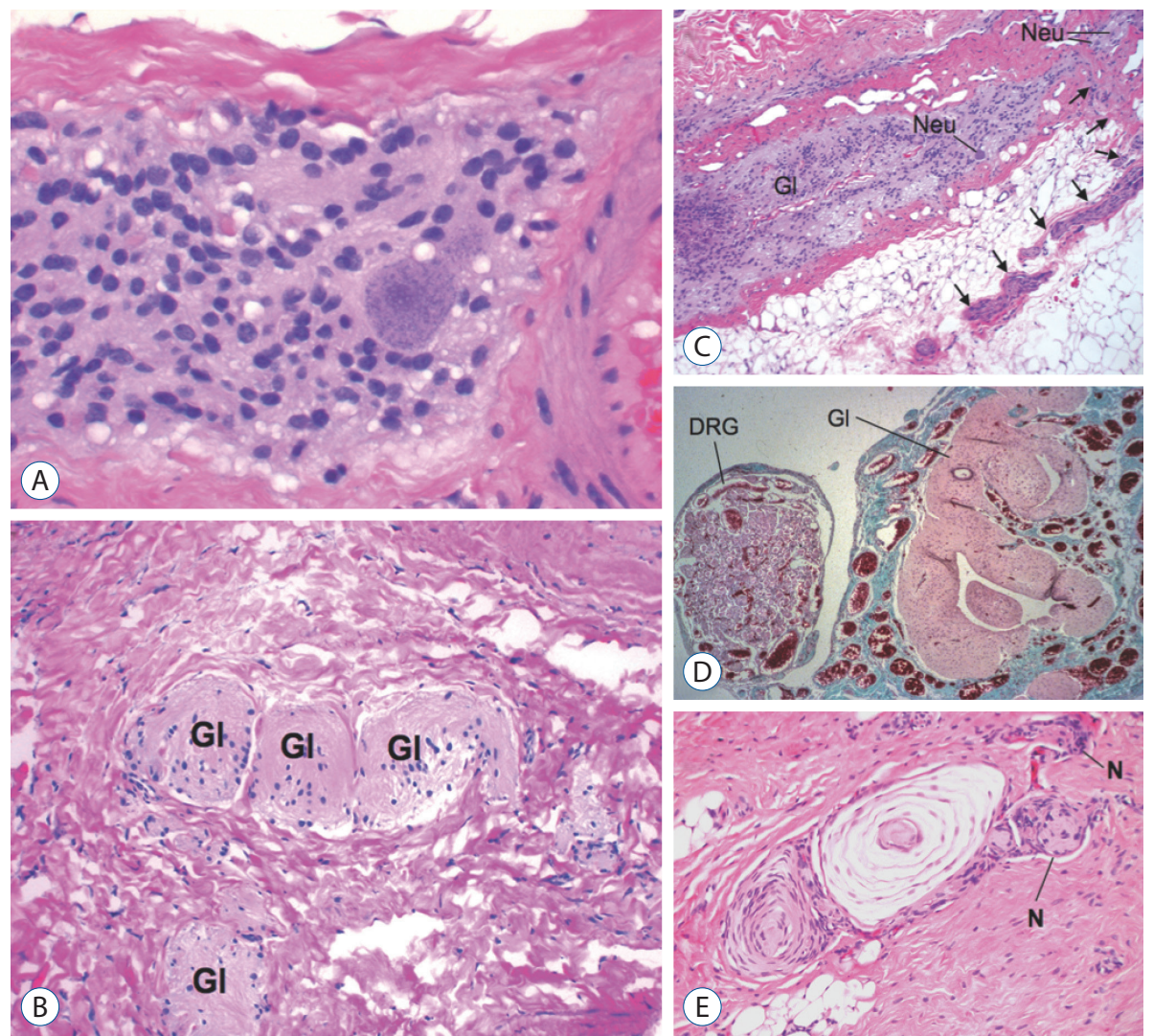

Fig. 26. Histopathology of limited dorsal myeloschisis (LDM) stalk (haematoxylin and eosin stain). A : Core of glial tissue with a large neuron, framed by fibrous tissue. B : Nests of glial tissue (Gl) embedded in a dense fibrous matrix. C : LDM stalk showing a longitudinal glial core (Gl) containing neurons (Neu). A peripheral nerve (arrows) issues forth at right angle to the glial core as from a "real" spinal cord. D : LDM stalk containing glial tissue (Gl) and a large dorsal root ganglion (DRG). E : Peripheral nerves (N) with Pacinian corpuscle within LDM stalk. Pacinian corpuscle in the stalk indicates the nerves involved in LDM formation are indeed sensory nerves likely from the adjacent neural crest. Reused from Pang et al. ${ }^{25)}$ with permission from Springer Nature. 

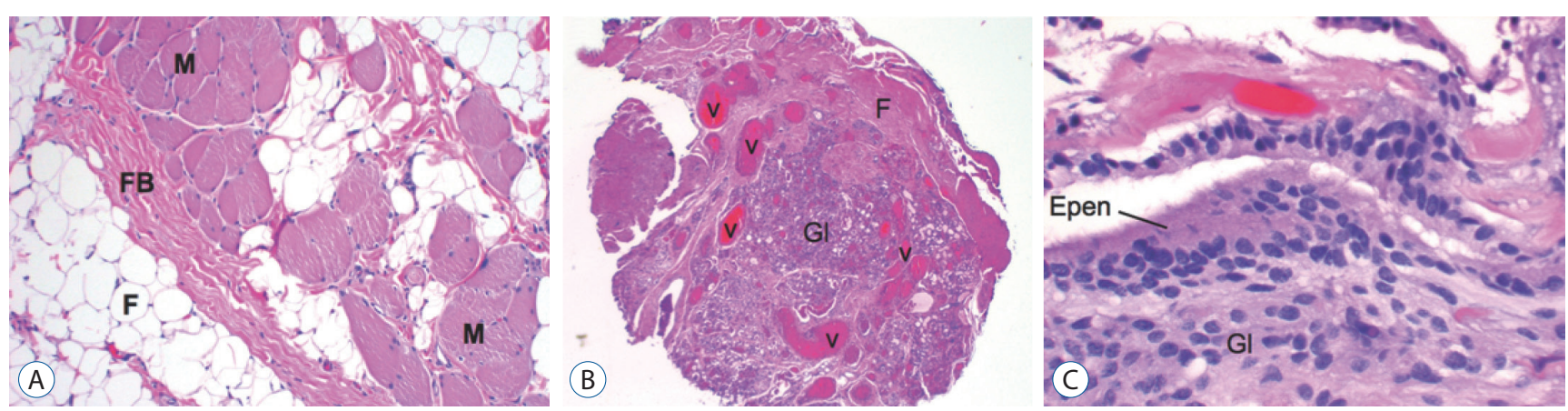

Fig. 27. Histopathology of limited dorsal myeloschisis (LDM) stalk (haematoxylin and eosin stain). A : LDM stalk containing skeletal muscle (M), fat (F), and fibrous band (FB). B : LDM stalk containing prominent blood vessels (V) within a core of glial tissue (GI), and fibrous bands (F), in the form of a vascular glomus. C : Glioependymal lining of a segmental myelocystocoele in a lumbar saccular LDM. Reused from Pang et al. ${ }^{25}$ ) with permission from Springer Nature. Epen : ependyma, Gl : glial tissue.

neural stalk passes through the lamina or penetrates the dura, and where it merges with the spinal cord. In the largest LDM series published ${ }^{25)}$, the vertebral level where the stalk merges with the spinal cord was chosen as the level of the LDM because it is usually the most unequivocal feature on MRI. The locations of the LDMs in that series are shown in Fig. 24, while the distribution of the types of LDMs is depicted in Fig. 25. Over two-thirds of LDMs in that series are located in the lower half of the spinal cord ${ }^{25}$.

Histologically, the central feature of all LDM stalks is neuroglial tissue, a hallmark of the stalk's origin from the NE. It is either in large elongated swaths containing scattered neurons (Fig. 26A), or in nests embedded in dense fibrous tissue (Fig. 26B). Also found in every stalk is a profuse network of peripheral nerves randomly admixed with the glial nests, but in some cases, nerves are seen emanating from a central core of neuron-containing glia likened to an abortive spinal cord (Fig. 26C). Large nodules of dorsal root ganglion cells are seen in some cases, attesting to the occasional entrapped neural crest stem cells during formation of the neural stalk (Fig. 26D). Pacinian corpuscles (Fig. 26E) seen amongst some of these nerves suggest they are indeed sensory axons. Evidence of mesenchymal condensation around the lengthening neural stalk is shown by the almost universal inclusion of numerous fibrous bands, skeletal muscle, fat (Fig. 27A), and prominent vascular channels sometimes in the form of a vascular glomus (Fig. 27B). Glioependymal tissue lines the sac cavities in cases of segmental myelocystocoeles (Fig. 27C). The cutaneous "cigarette-burn mark" has the histological appearance of a dermal layer with engorged vascularity, abundant nerve fibres, and an abnormal collagen fiber matrix. The unevenness of its surface is

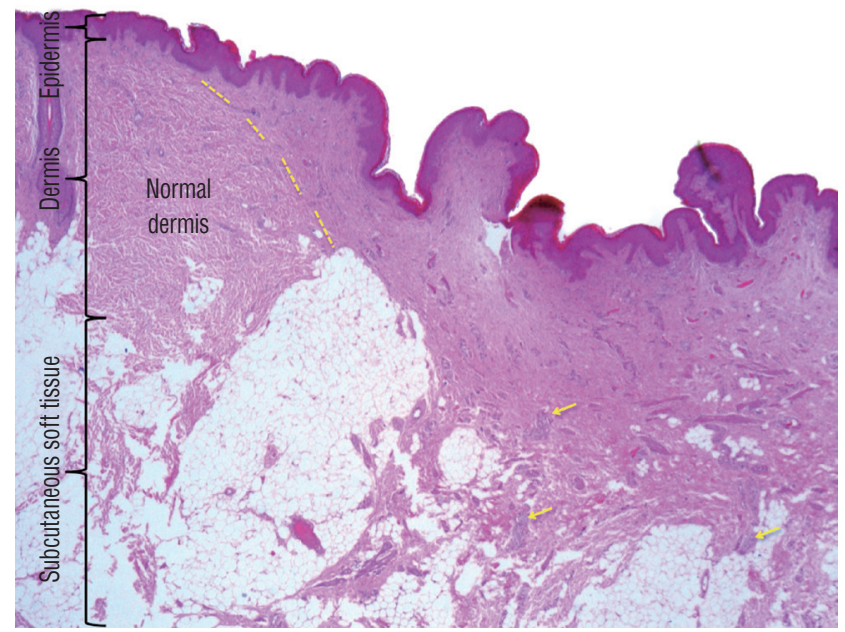

Fig. 28. IHistological slide of a skin "cigarette burn mark" showing increase vascularity, plenty of nerve fibres (yellow arrows), and a different collagen pattern, comparing to the adjacent normal dermis. Yellow dashed line marks the border between normal and abnormal dermis. Gross appearance of the skin lesion is shown in Fig. 29A (haematoxylin and eosin stain). Reused from Wong et al. ${ }^{43)}$ with permission from Springer Nature.

due to the ruggedness of the epidermis and dermis (Fig. 28) ${ }^{16,17)}$.

The question is sometimes asked what constitute the minimum criteria for a diagnosis of LDM. It has been shown that there are patients with clinical and radiological features of LDM (Figs. 29 and 30) and most of its histological features including periphery nerve fibers, but no glial tissue within the

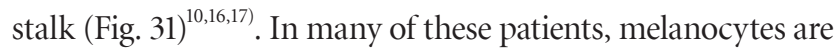
also a prominent feature. Since periphery nerves and melanocytes are neural crest derivatives, and neural crest cells are located in the primary neural tube over the dorsal midline, a nondisjunctional stalk might drag with it neural crest progen- 


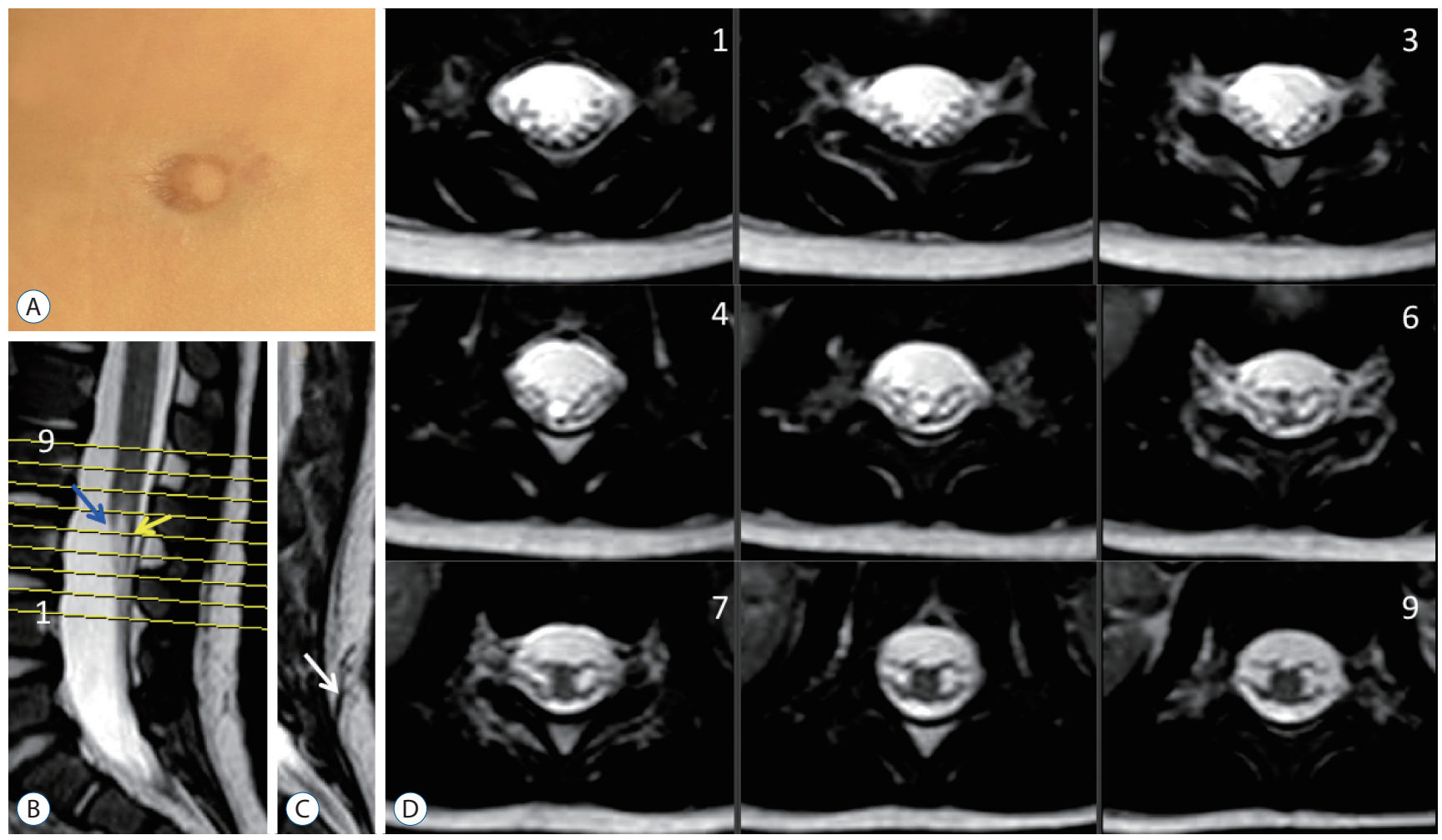

Fig. 29. A case of "clinical and radiolgical" limited dorsal myeloschisis (LDM). A : Cigarette burn mark. B : Mid-sagittal T2 weighted MRI image showing the intradural portion of the LDM stalk (yellow arrow). Blue arrow means the conus. C : Sagittal MRI just next to B showing the subcutaneous portion of the stalk (white arrow). D : Axial T2-weighted MRI images corresponding to the cut lines in (B). Reused from Wong et al. ${ }^{43)}$ with permission from Springer Nature. MRI : magnetic resonance imaging.
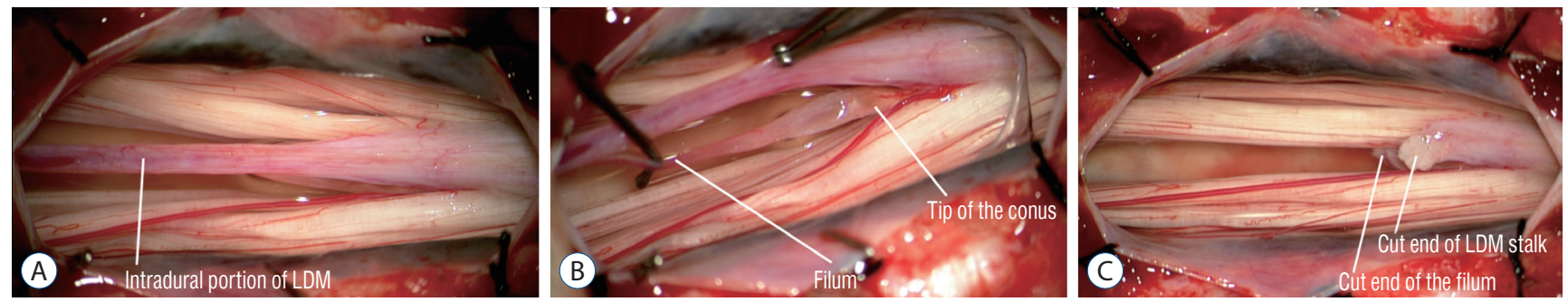

Fig. 30. Intra-operative photographs showing excision of a LDM with a L2L3 laminoplasties. Pre-operative MRI images and the skin lesion are shown in Fig. 29. The skin lesion was traced to the supraspinous ligament of S1 only; the S1 laminae were untouched. This kind of limited exposure thus left a small segment of the LDM stalk in situ. Reused from Wong et al. ${ }^{43)}$ with permission from Springer Nature. LDM : limited dorsal myeloschisis, MRI : magnetic resonance imaging.

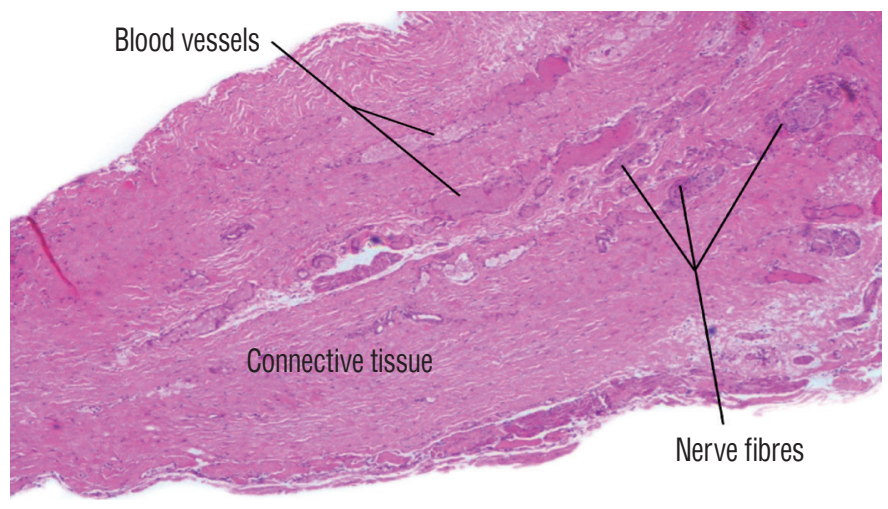

Fig. 31. Histological slide of the intradural portion of a limited dorsal myeloschisis stalk showing the presence of nerve fibres and blood vessels. Intraoperative photograph is shown in Fig. 30 (haematoxylin and eosin stain). Reused from Wong et al. ${ }^{43)}$ with permission from Springer Nature. 
itor cells without neuroglial progenitor cells ${ }^{2,30,31,37)}$. Thus the diagnosis of LDM can probably be applied in these patients with periphery nerve fibers but no glioneuronal tissue in the stalk.

\section{Clinical manifestations}

Most LDM patients also present at a young age. In a series with a total of 63 patients, the mean age at presentation of 56 children was 5.9 years; and that of seven adults was 28.2 years.

About half of LDM patients are neurologically intact at presentation, which underscores the importance of the cutaneous marker as an initial diagnostic clue ${ }^{25)}$. The "pathognomic" cutaneous marker in both flat and saccular LDMs is a confined
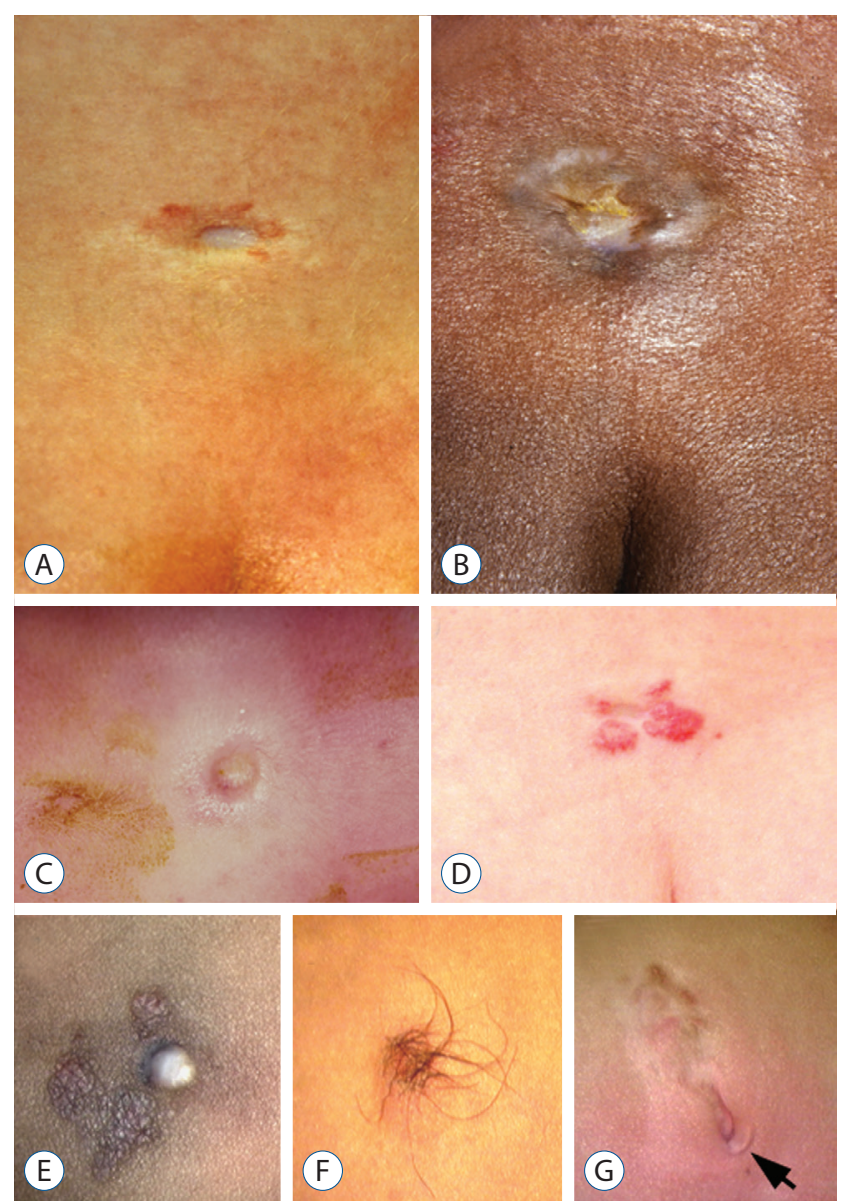

Fig. 32. Flat type skin lesions in limited dorsal myeloschisis. A : Sunken crater of pale squamous epithelium. B : Sunken crater of pale epithelium. C : Squamous epithelial crater with rim of elevated skin borders. D : Crater surrounded by prominent capillary haemangioma with irregular corrugated borders. E : White non-melanotic, epithelial crater with surrounding hyperpigmented skin. F : Crater covered with long hair arising from the rim of surrounding full-thickness skin. G : Crater with surrounding skin overhang (arrow). Reused from Pang et al. ${ }^{25)}$ with permission from Springer Nature. area of abnormal epithelium over the dorsal midline. In flat LDMs, the cutaneous lesion can be a conspicuous crater or a tiny pit. 1) Crater : the commonest skin abnormality in flat LDMs is a sunken crater on the flat skin surface made of pinkish squamous epithelium (Fig. 32A and B), often with elevated skin margin (Fig. 32C) and sometimes surrounded by a capillary haemangioma with irregular corrugated borders (Fig. 32D) or hyperpigmented skin (Fig 32E). There are occasionally long hair emanating from the crater (Fig. 32F), and some craters are edged by hooded overhanging skin (Fig. 32G).
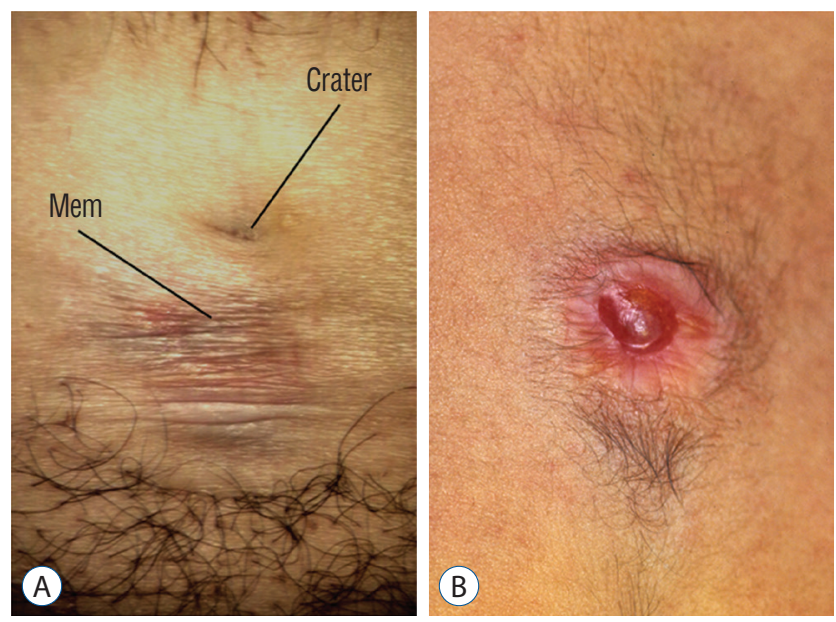

Fig. 33. Flat LDMs with transitional skin lesions: A : Lumbar LDM with a flat epithelial crater and an adjacent area made of stretchable, non-skin epithelium (Mem) that distends into a small CSF-filled bubble when the patient strains. B : Pink epithelial crater slightly distended into a small blister by underlying CSF. Reused from Pang et al. ${ }^{25)}$ with permission from Springer Nature. LDM : limited dorsal myeloschisis, CSF : cerebrospinal fluid.

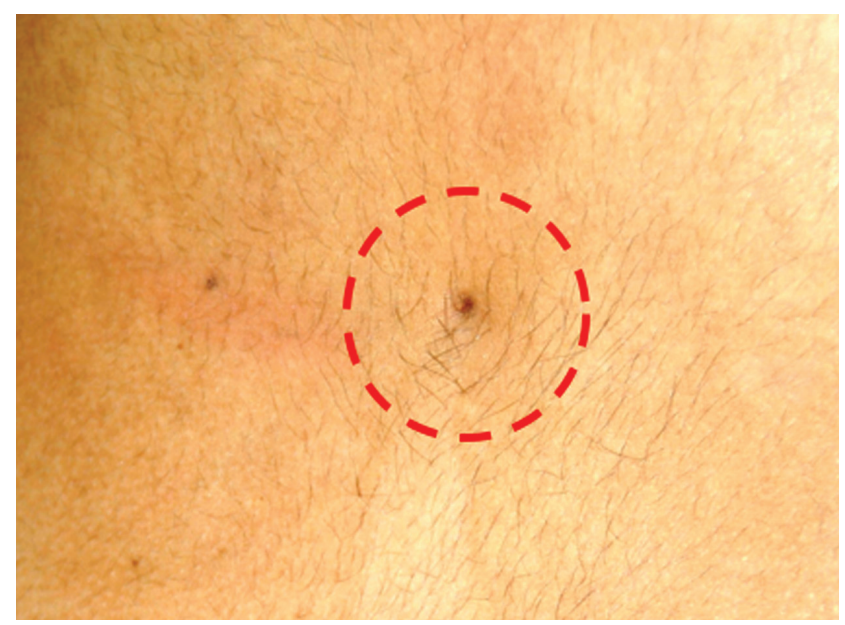

Fig. 34. Subtle pit (within circle) in a flat lumbar limited dorsal myeloschisis with no surrounding exuberance. Reused from Pang et al. ${ }^{25)}$ with permission from Springer Nature. 
In several examples, the crater is adjacent to an area of wrinkly, over-stretched skin that periodically distends with CSF on dependent posturing or straining (Fig. 33A). Very rarely, the centre of the crater is adorned with a CSF-filled blister (Fig. $33 \mathrm{~B}$ ), which is transitional form between flat and saccular lesions. And 2) pit : the most subtle skin abnormality in a flat LDM is a small midline pit with no other unusual features (Fig. 34), easily missed on cursory examination, and might be confused with a CSDST ostium. Sometimes the pit situates within a capillary haemangioma. Pit lesions are usually found in low thoracic and lumbar cases.

Externally, saccular LDMs usually appear as a skin-based sac, but rarely as a translucent membranous sac. 1) Skin-based sac : the sac wall is thick, skin-based with a dome that is distinctly abnormal skin. The appearance of the dome cover can be roughly subdivided into three subtypes. One subtype has a wide top of purplish, raw-looking, thick stratified squamous epithelium (Fig. 35A). A second subtype has a much smaller, pale, discrete, puckered crater of squamous epithelium on the dome (Fig. 35B). A third subtype has a small, almost imper- ceptible patch of ultra-thin epithelium on the apex of the relatively delicate skin-based dome (Fig. 35C). And 2) membranous sac : this type should be managed urgently as an "open" spinal dysraphic lesion, but the fibroneural stalk should not be missed. An example is a tubular, CSF-filled sac made of a diaphanous membrane resembling thickened arachnoid, protruding through a $4 \mathrm{~mm}$ skin-lined dorsal defect (Fig. 36). The base of this sac has a shallow collar of skin similar to the skinbased sacs. The locations and types of the cutaneous lesions are summarized in Table 1.

It is noteworthy that other cutaneous markers of dysraphism such as hypertrichosis, capillary haemangioma, or misaligned gluteal crease are never seen alone in LDM without the quintessential epithelial crater or pit. The pearly midline crater in a non-saccular LDM thus remains the single most important diagnostic clue for LDM, especially before the development of neurological symptoms.

LDMs cause neurological deficits solely by their tethering effect, which vary in kind and severity by the spinal level of the LDM. In general, LDM patients tend to have milder dis-
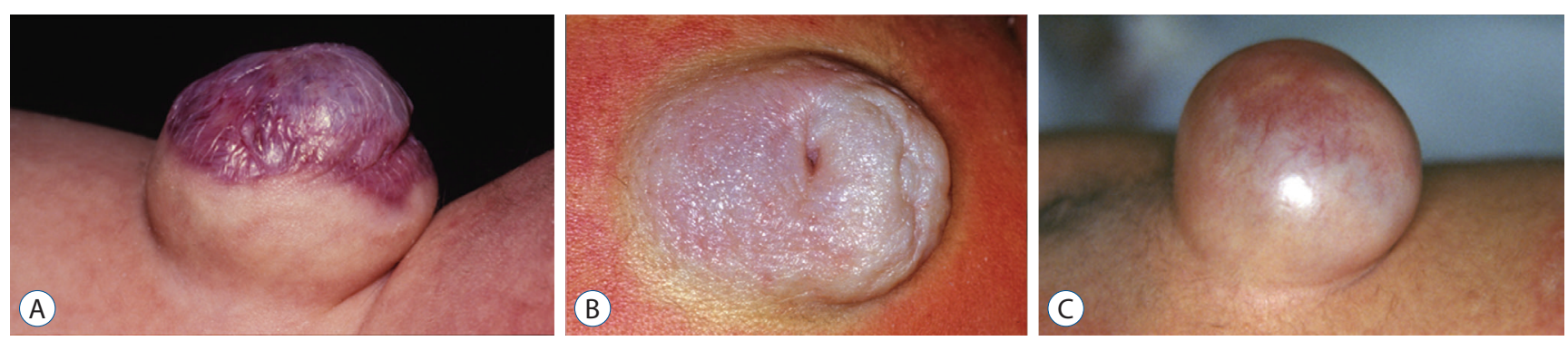

Fig. 35. Saccular skin lesions in limited dorsal myeloschisis (LDM). A : A cervical saccular LDM with full-thickness skin at the base and coarse, thick, corrugated purplish squamous epithelial top. Cervical saccular lesions are usually not turgid. B: An upper thoracic saccular LDM with mostly skin except for a dome crater of squamous epithelium. C : A turgid lumbar saccular LDM with a skin base and a translucent "non-skin" epithelial top. Reused from Pang et al. ${ }^{25)}$ with permission from Springer Nature.
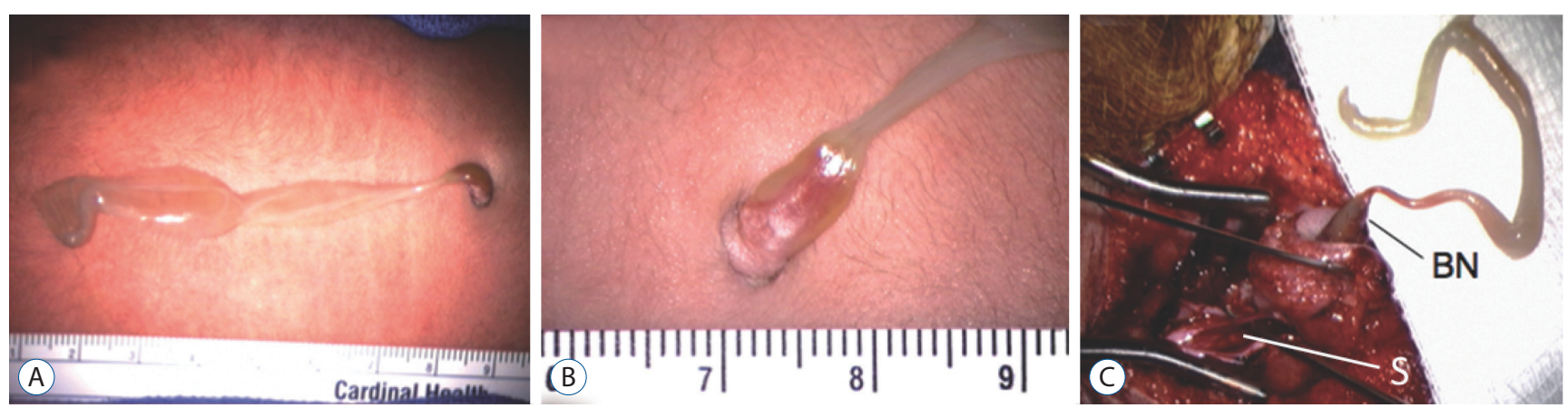

Fig. 36. Lumbar limited dorsal myeloschisis (LDM) with membranous sac. A : Large ruptured sac made of diaphanous membrane. B : Close-up of the base showing a small skin defect through which protrudes a tubular basal neural nodule. $C$ : The entire LDM is exposed at surgery to show basal neural nodules (BN), subcutaneous tract, and intradural stalk (S) attached to the spinal cord. Reused from Pang et al. ${ }^{25)}$ with permission from Springer Nature. 
ability than patients with other forms of dysraphic malformations such as split cord malformations and spinal cord lipomas. In Pang et al.'s series ${ }^{25)}$, half of the LDM patients had neurological deficits at presentation. About $10 \%$ of patients had significant weakness and neuropathic bladder, and the rest of the patients had mild or tolerable neurological or urological deficits, with relatively little hindrance to their lifestyle. The correlations between the types of deficits and locations of the LDMs are summarized in Table 2. The proximity of tension to the relevant cord segments does seem to correspond with the kind of deficits. For example, only cervical lesions produce hand and arm weakness; leg weakness is seen in only $9 \%$ of cervical lesions, but $22 \%$ in upper thoracic lesions, $38 \%$

Table 1. Skin lesions in LDM in different LDM locations $(n=63)$

\begin{tabular}{lcccc}
\hline & \multicolumn{4}{c}{ Skin Lesions in LDM } \\
\cline { 2 - 5 } LDM location* & Crater & Pit & Saccular & $\begin{array}{c}\text { Membranous } \\
\text { sac }\end{array}$ \\
\hline Cervical & 2 & 0 & 9 & 0 \\
Thoracic-upper & 3 & 2 & 4 & 0 \\
Thoracic-lower & 4 & 0 & 3 & 0 \\
Thoracolumbar & 4 & 2 & 2 & 0 \\
Lumbar & 16 & 4 & 6 & 2 \\
\hline
\end{tabular}

Reused from Pang et al. ${ }^{25)}$ with permission from Springer Nature. *The regions of the vertebral column are coded as: cervical (C1-C7); thoracicupper (T1-T5); thoracic-lower (T5-T11); thoracolumbar (T12-L1); and lumbar (L1-L5). LDM : limited dorsal myeloschisis in thoracolumbar lesions, and 50\% in lumbar lesions; and bladder dysfunction is seen in approximately 15\% of lower thoracic and lumbar lesions but not in cervical or upper thoracic lesions. Lumbar LDMs close to the conus are perhaps more treacherous because they more often implicate the bladder yet are more likely to be occult.

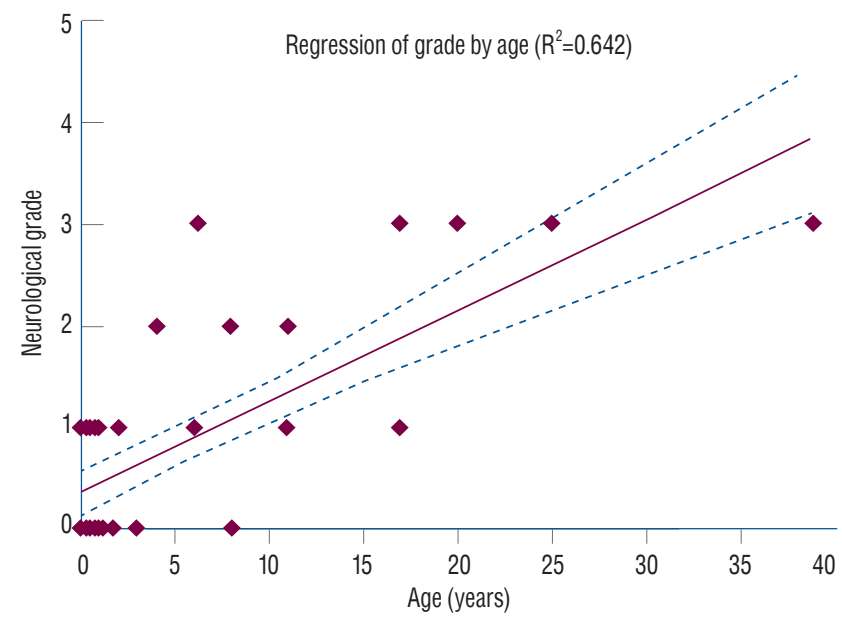

Fig. 37. Linear regression analysis between neurological grade* and patient age shows a logistical tendency for older patients with limited dorsal myeloschisis (LDM) to present with higher grades of neurological deficits (correlative coefficient $R^{2}=0.642$ ). Reused from Pang et al. ${ }^{25)}$ with permission from Springer Nature. *Neurological grading system in LDM : grade 0 , no deficits or symptoms; grade 1 , mild upper or lower extremity weakness, or pure sensory deficits \pm pain; grade 2, moderate to severe upper or lower extremity weakness \pm sensory deficits, or neurogenic bladder without weakness; grade 3, upper or lower extremity weakness+neurogenic bladder.

Table 2. Types of neurological deficits in different LDM locations $(n=63)$

\begin{tabular}{|c|c|c|c|c|c|}
\hline \multirow[b]{2}{*}{ Neurological status } & \multicolumn{5}{|c|}{ Number of patients assorted by LDM location } \\
\hline & $\begin{array}{l}\text { Cervical } \\
(n=11)\end{array}$ & $\begin{array}{l}\text { Thoracic upper } \\
\qquad(n=9)\end{array}$ & $\begin{array}{l}\text { Thoracic lower } \\
\qquad(n=7)\end{array}$ & $\begin{array}{l}\text { Thoracolumbar } \\
\qquad(n=8)\end{array}$ & $\begin{array}{c}\text { Lumbar } \\
(n=28)\end{array}$ \\
\hline Normal & $4(36.0)$ & $2(22.0)$ & $4(57.0)$ & $4(50.0)$ & $14(50.0)$ \\
\hline UE weakness/sensory loss & $7(64.0)$ & $2(22.0)$ & & & \\
\hline LE weakness & $1(9.0)$ & $2(22.0)$ & $3(43.0)$ & $3(38.0)$ & $14(50.0)$ \\
\hline LE sensory loss & & $1(11.0)$ & $2(31.0)$ & $1(13.0)$ & $8(29.0)$ \\
\hline Spastic legs & $4(36.0)$ & $4(44.0)$ & $2(31.0)$ & & \\
\hline Back pain & & & $1(25.0)$ & & $3(13.6)$ \\
\hline Foot deformity & & & & & $2(9.1)$ \\
\hline Scoliosis & & & $2(31.0)$ & $1(16.6)$ & $1(4.5)$ \\
\hline Neurogenic bladder & & & $1(25.0)$ & $1(16.6)$ & $4(18.0)$ \\
\hline Abnormal URD & & & $1(25.0)$ & $1(16.6)$ & $2(9.1)$ \\
\hline
\end{tabular}

Values are presented as number (\%). Reused from Pang et al. ${ }^{25)}$ with permission from Springer Nature. LDM : limited dorsal myeloschisis, UE : upper extremity, LE : lower extremity, URD : urodynamics 
Similar to other cord tethering entities, the probability of neurological injury increases with longitudinal growth of the spine and with age. Pang et al. ${ }^{25)}$ demonstrated that older patients with LDMs had a tendency to present with more severe neurological and urological disabilities; and that infants and young children were more likely to be neurologically normal (Figs. 37 and 38). Neurological deterioration was observed in all four adolescents who had had longitudinal follow-up and delay of surgery of 1 to 9 years.

\section{Mixed LDM and CSDST, LDM with hidden der- mal elements, and parallel LDM and CSDST in close proximity}

In 2013, lesions definitely composed of the histological find-

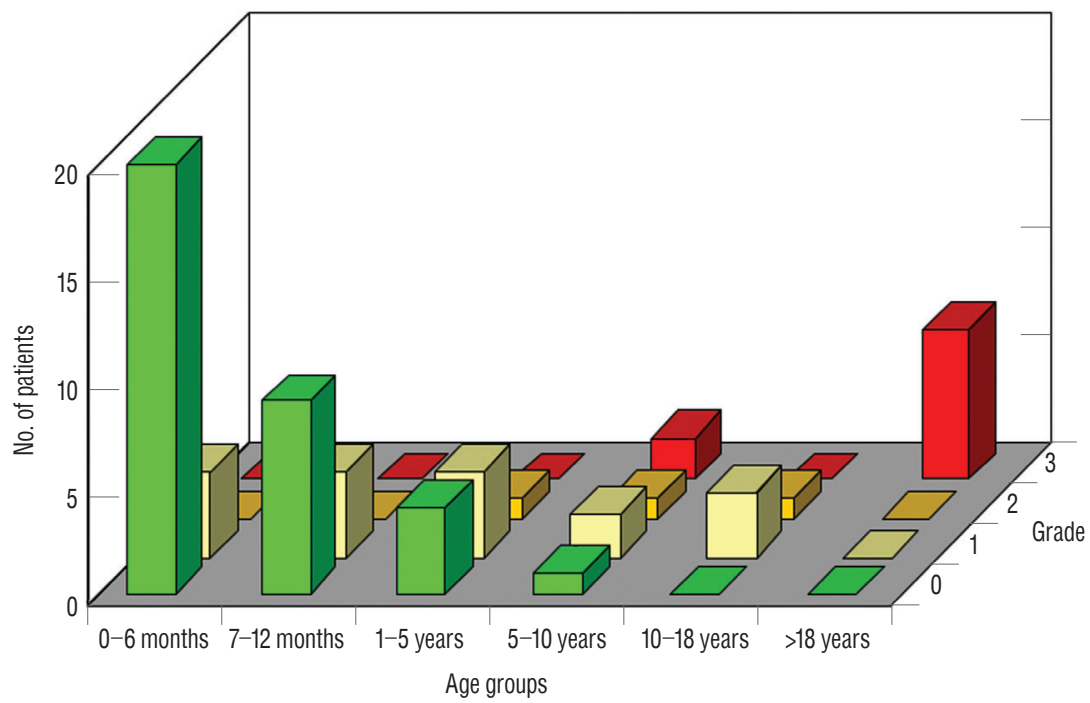

Fig. 38. Clustered bar graphs showing neurological grade assorted by patients' age-groups (birth to 6 months; 6 to 12 months; 1 to 5 years; 6 to 10 years; 11 to 18 years; and over 18 years) within each neurological grade of $0-3$. There is a preponderance of younger children with the better neurological grades and preponderance of older patients with the worse grades. Reused from Pang et al. ${ }^{25}$ with permission from Springer Nature.

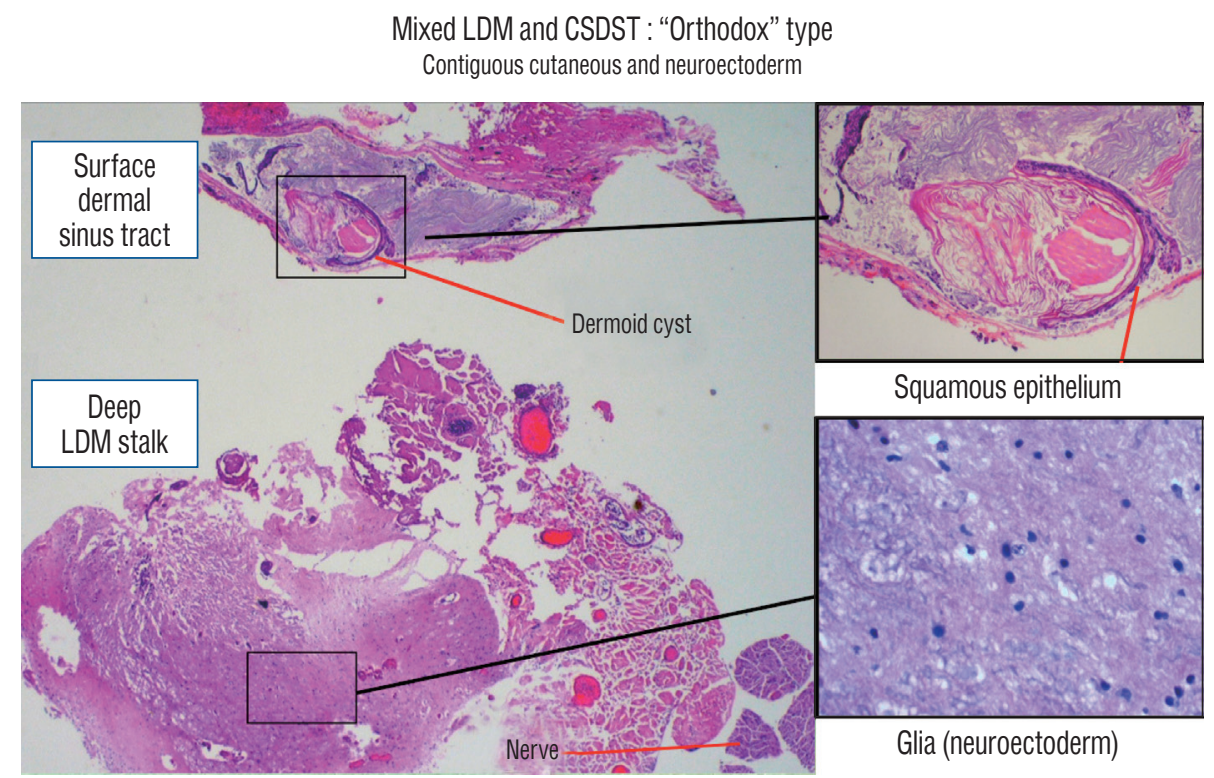

Fig. 39. Histological constituents of a mixed LDM-CSDST stalk of the "orthodox" type. Its superficial portion is of typical dermal tissue lying in tandem with its deeper portion containing mainly glioneuronal tissue. Insets showing both tissues in high power (haematoxylin and eosin stain). LDM : limited dorsal myeloschisis, CSDST : congenital spinal dermal sinus tract. 

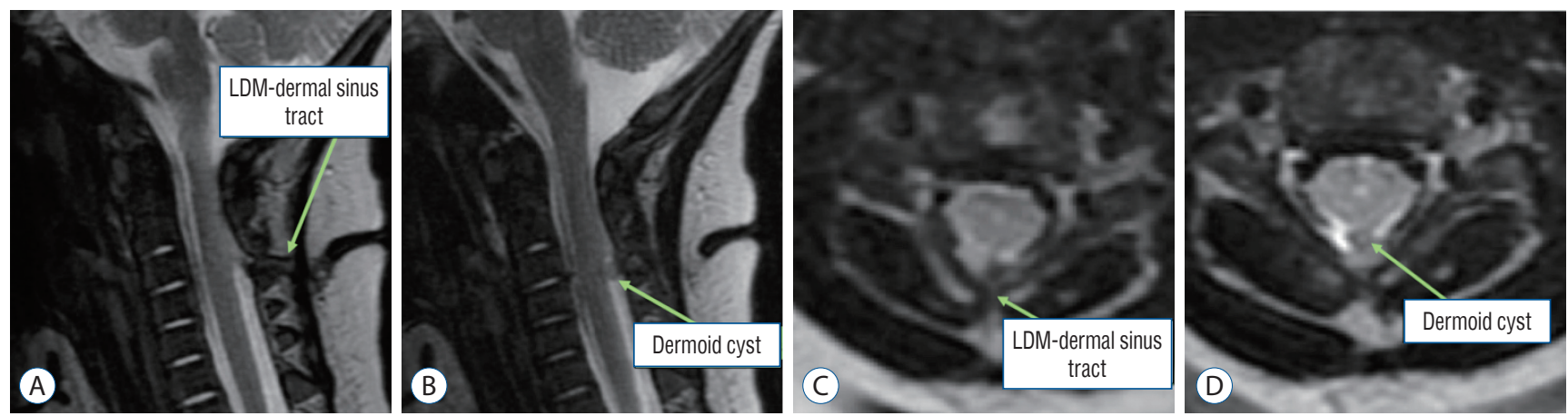

Fig. 40. MRI images of a 3-month-old girl with a mixed LDM and CSDST - "conjoint" type. The diagnosis was made based on intraoperative and histological findings (Figs. 41 and 42). MRI images can only show a tract extending from the skin to the spinal cord, but cannot confirm the components of the tract. A and B : Sagittal T2-weighted MRI images. C and D : Axial T2-weighted MRI images. LDM : limited dorsal myeloschisis, CSDST : congenital spinal dermal sinus tract, MRI : magnetic resonance imaging.
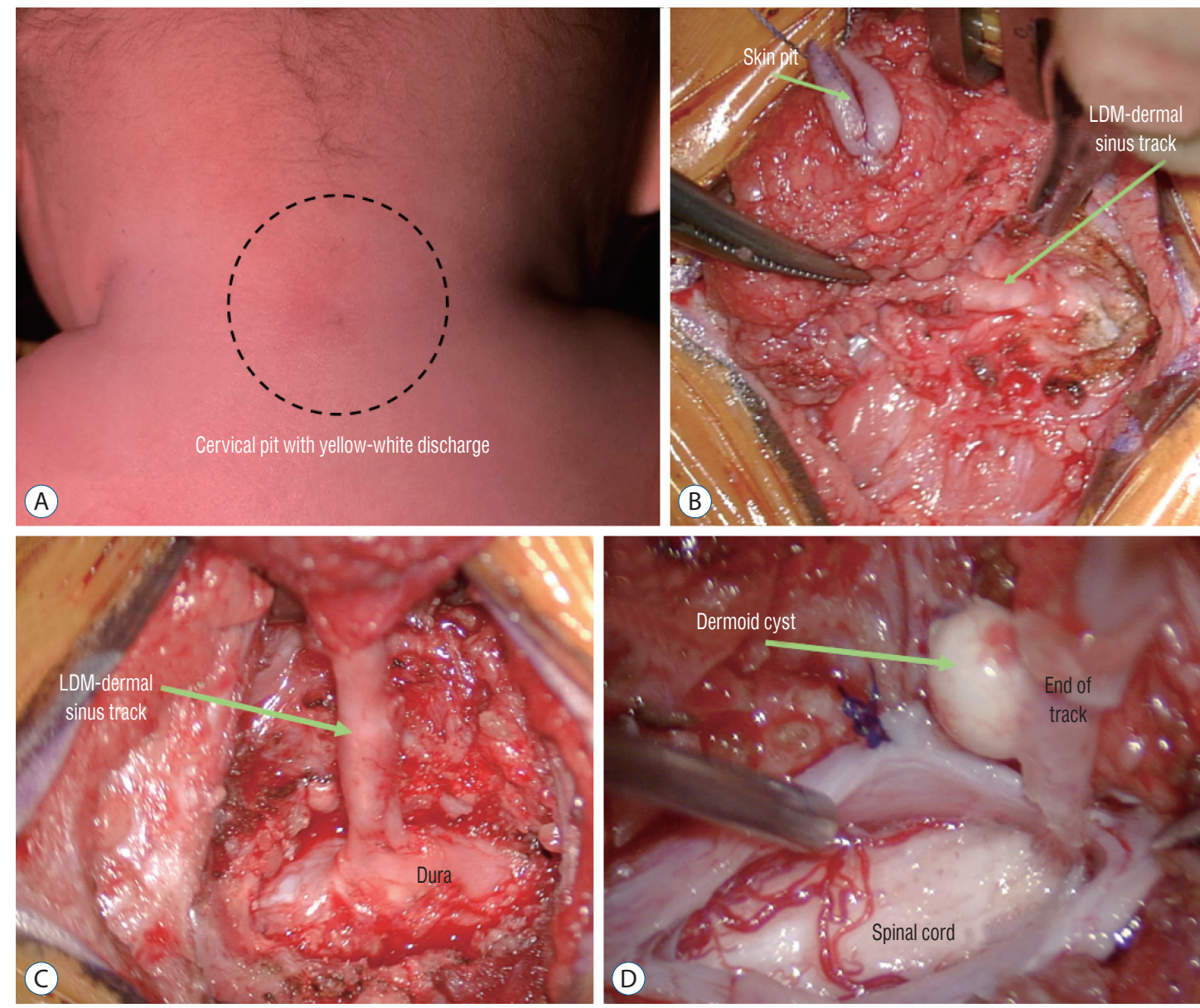

Fig. 41. Intraoperative photos of a patient (Fig. 40) with a mixed LDM and CSDST - "conjoint" type. A : Skin pit. B and C : Dissection of the extra-dural portion of the tract. D : Photo taken at the moment before complete detachment of the tract from the spinal cord. LDM : limited dorsal myeloschisis, CSDST : congenital spinal dermal sinus tract.

ings of both LDMs and CSDSTs were first described ${ }^{25}$. They are rare compared to the pure forms of LDMs and CSDSTs. In a series of $75 \mathrm{LDM}$ cases, there were five cases of this mixed type ${ }^{5}$. In another series of 51 cases that consisted of 40 LDMs and 11 CSDSTs, another five cases were documented ${ }^{111}$.

Macroscopically, a mixed lesion of LDM and CSDST can 
Dermal sinus tract within fibro-glioneuronal stalk
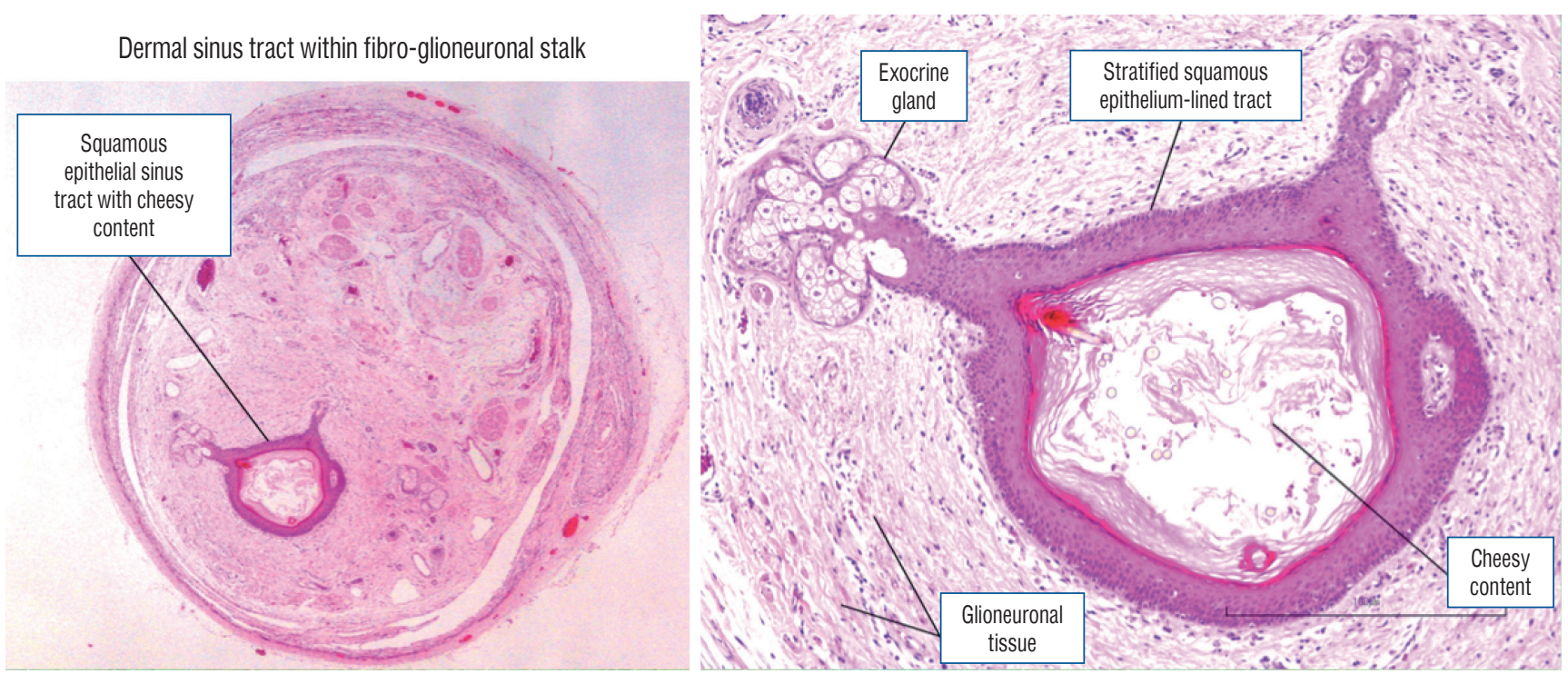

Fig. 42. Histological slides of a patient (Fig. 40) with a mixed LDM and CSDST - "conjoint" type showing the presence of a dermal sinus tract within a fibro-glioneuronal stalk (haematoxylin and eosin stain). LDM : limited dorsal myeloschisis, CSDST : congenital spinal dermal sinus tract.
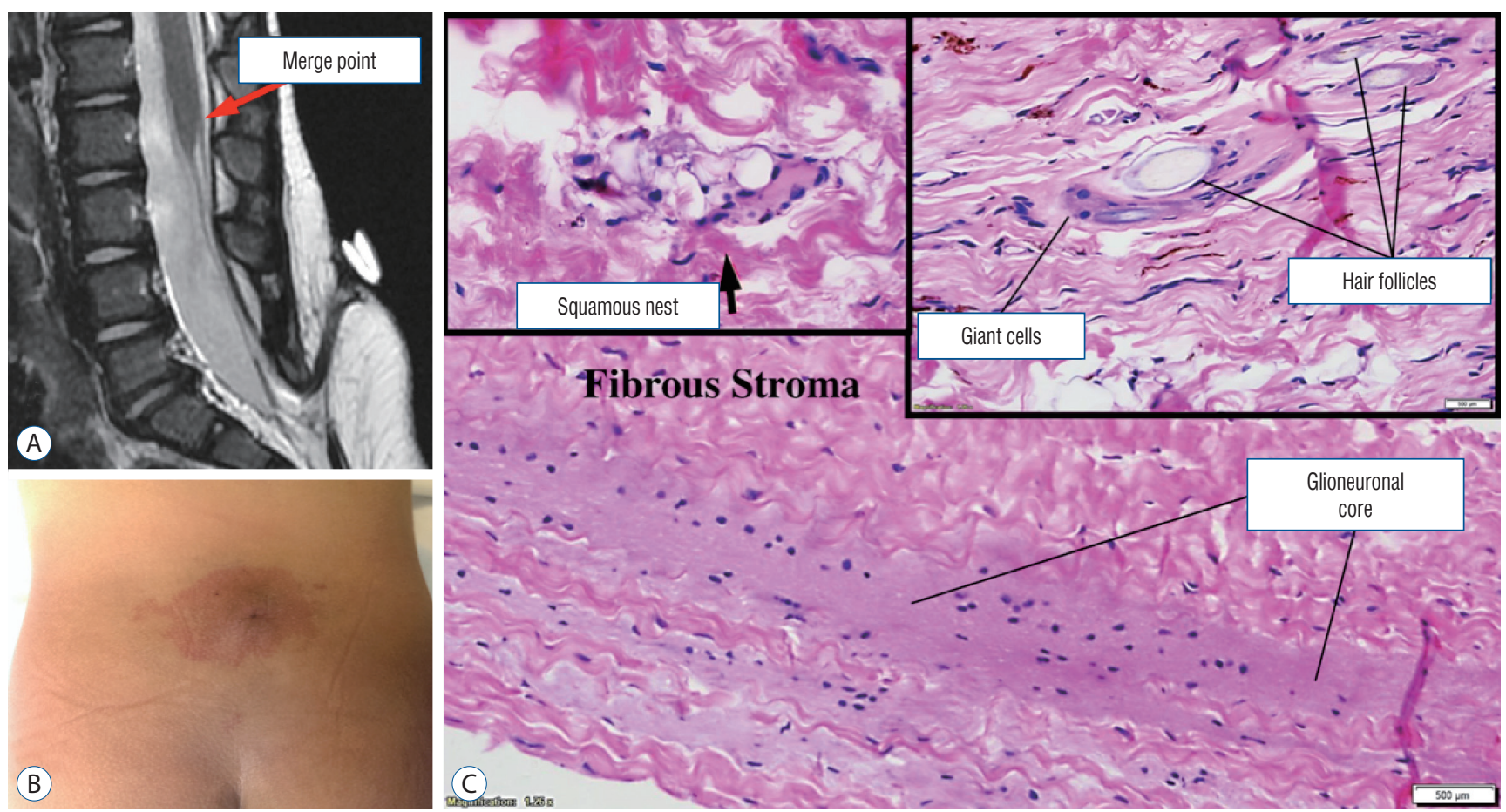

Fig. 43. A 15-month-old with a LDM with hidden dermal elements. A : Sagittal T2-weighted MRI showing the appearance of a classic lumbar LDM. B : Photo showing a crater and surrounding haemangioma. C : Histological slide showing a stalk with glioneuronal core and derivatives of squamous epithelium (haematoxylin and eosin stain). LDM : limited dorsal myeloschisis, MRI : magnetic resonance imaging.

mimic either of the pure forms. Even cystic type has been observed $^{11)}$. Thus, their recognition relies on histology. Three histological types have been documented. In the "orthodox" type, the dermal and neuroglial elements are in tandem in their respective embryologically orthodox order, i.e., an outer tract of CSDST and an inner tract of LDM elements (Fig. $39)^{11}$. In the "conjoint" type, the entire FSND tract is lined by both SE and NE elements (Figs. 40-42). The most treacherous 
Parallel LDM stalk and dermal sinus tract
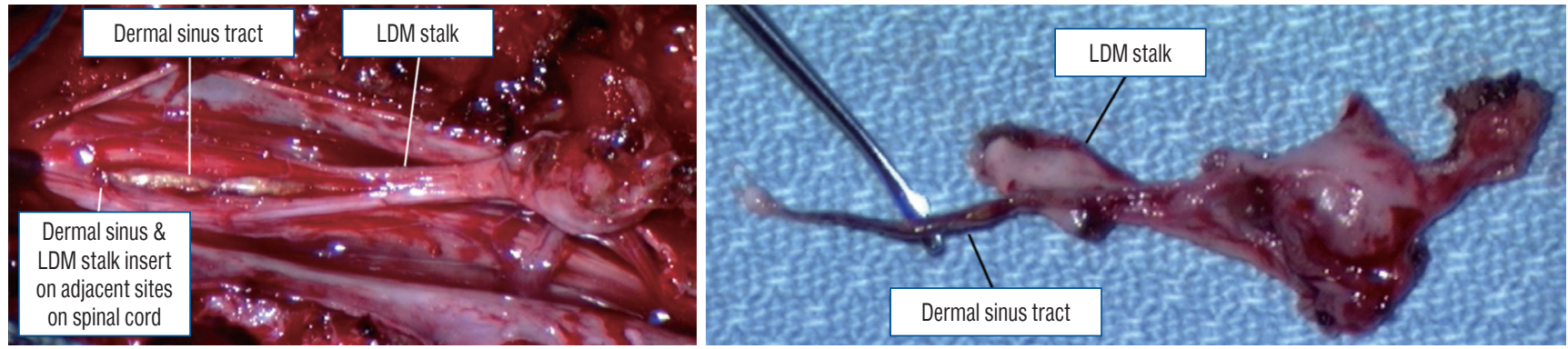

Fig. 44. Intraoperative photos showing the presence of a LDM and a CSDST in parallel. LDM : limited dorsal myeloschisis, CSDST : congenital spinal dermal sinus tract.

Table 3. Associated anomalies in LDM ( $n=63)$

\begin{tabular}{|c|c|c|c|c|c|}
\hline \multirow[b]{2}{*}{ Anomalies } & \multicolumn{5}{|c|}{ LDM location } \\
\hline & $\begin{array}{l}\text { Cervical } \\
(n=11)\end{array}$ & $\begin{array}{l}\text { Thoracic upper } \\
\qquad(n=9)\end{array}$ & $\begin{array}{l}\text { Thoracic lower } \\
\qquad(n=7)\end{array}$ & $\begin{array}{l}\text { Thoracolumbar } \\
\qquad(n=8)\end{array}$ & $\begin{array}{c}\text { Lumbar } \\
(n=28)\end{array}$ \\
\hline SCM & 4 & 1 & & & 1 \\
\hline Terminal lipoma & & 1 & & & 1 \\
\hline Dorsal lipoma & & 1 & & 2 & 3 \\
\hline Thickened filum & 2 & 1 & & 3 & 21 \\
\hline Neurenteric cyst & & & & & 1 \\
\hline Syringomyelia & & 1 & & 1 & \\
\hline Chiari II & 3 & 2 & & & \\
\hline Hydrocephalus & 6 & & & & \\
\hline Dermal sinus & & & 1 & 1 & 1 \\
\hline Velum interpositum cyst & & 1 & & & \\
\hline Vertebral/rib fusion & & 2 & 1 & & \\
\hline
\end{tabular}

Reused from Pang et al. ${ }^{25)}$ with permission from Springer Nature. LDM : limited dorsal myeloschisis, SCM : split cord malformation

mixed lesions have been observed in which the dermal elements form microscopic squamous epithelial islands within the neuroglial tissue of an otherwise proper LDM tract "LDM with hidden dermal elements" (Fig. 43) ${ }^{5,38)}$. Rarely, parallel LDM and CSDST tracts can co-exist in close proximity from skin to spinal cord (Fig. 44).

The most salient implication in clinical practice with the discovery of this mixed type is that a mixed lesion cannot be reliably exonerated at the time of surgery without benefit of histology. Thus, it seems prudent that in all cases of suspected $\mathrm{LDM}$, the entire tract is removed from skin to spinal cord. In addition, all FSND patients should have a delayed post-operative MRI to rule out a recurrent dermoid cyst.
FSND with spinal cord lipomas, split cord malformations, and other dysraphic malformations

FSNDs have been observed to occur with other dysraphic or paradysraphic malformations such as spinal cord lipomas, myelomeningoceles, split cord malformations, and neurenteric cysts (Table 3 and Fig. 45). In such cases, the complex anatomy of the other malformations usually dominates the pathological anatomy of the composite malformation, for a pure FSND lesion is structurally more subtle unless it is a CSDST with a large intradural dermoid/ epidermoid cyst.

The clinical manifestations of these composite malformations also follow the usual course of the associated anomalies, especially when there are only LDM elements without dermoid cyst. However, if present, the dermal elements can have 

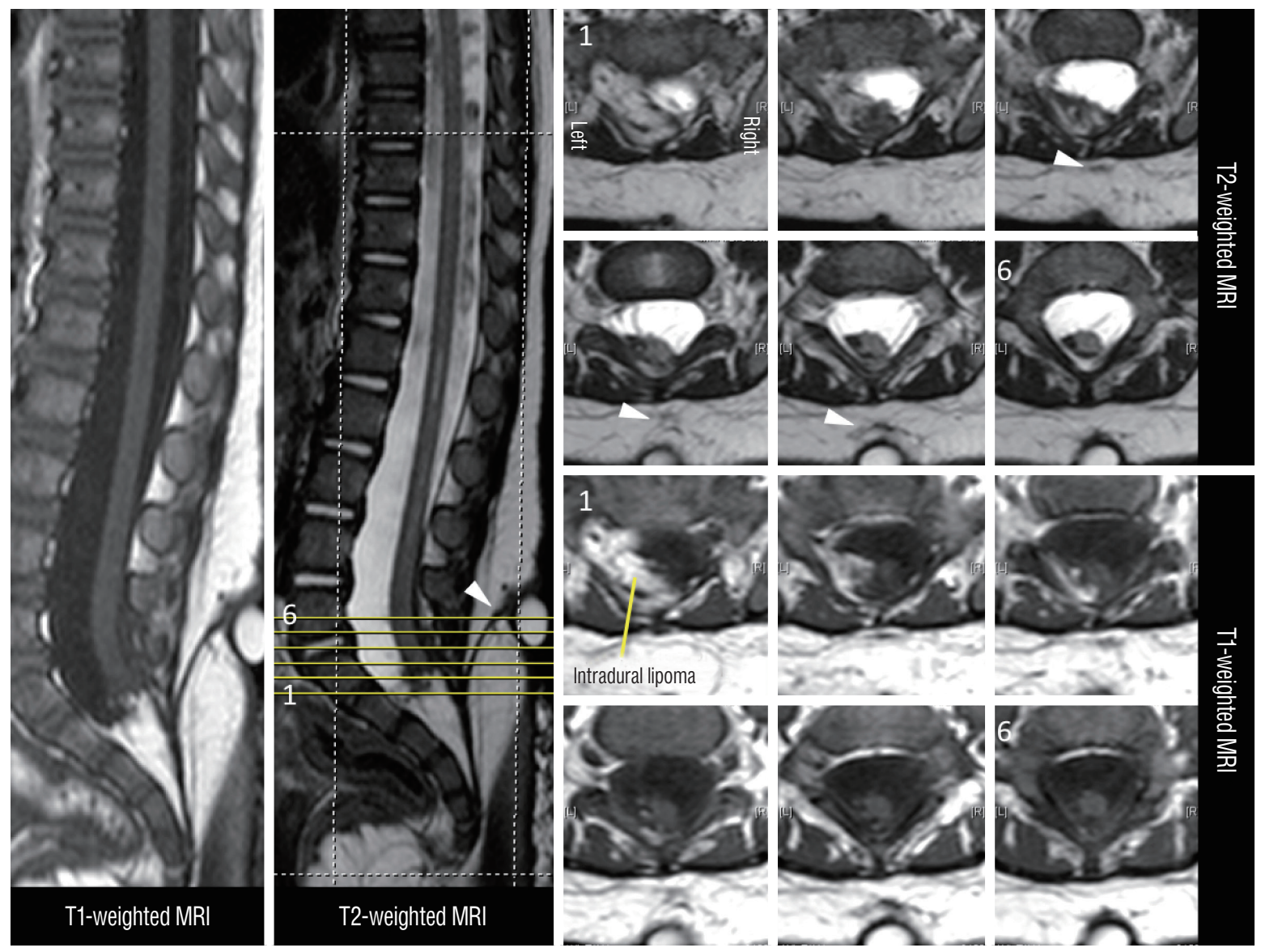

Fig. 45. MRI images showing a LDM associated with a transitional spinal cord lipoma in an 11-month-old. The axial cuts are numbered according to the cut-lines on the T2-weighted mid-sagittal MRI image. Arrowheads mean the subcutaneous portion of the LDM stalk. The stalk was confirmed intraoperatively to pass through bifid S1 and S2 laminae. Reused from Wong et al. ${ }^{43}$ with permission from Springer Nature. MRI : magnetic resonance imaging, LDM : limited dorsal myeloschisis.

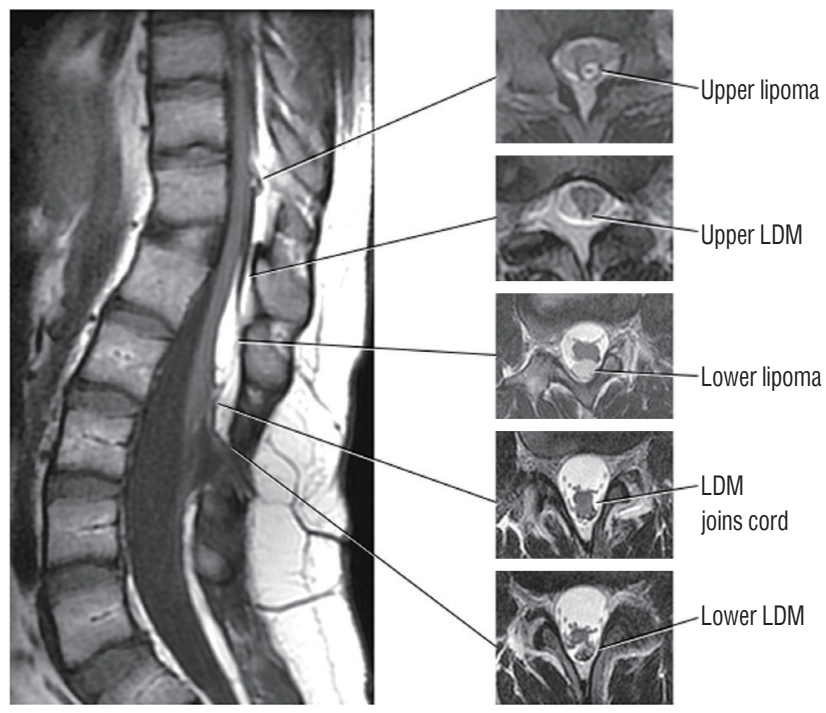

Fig. 46. Double LDMs, both crater type, with accompanying dorsal lipomas. The lower LDM is at $\mathrm{L}_{2 / 3}$, and upper LDM is at $\mathrm{T}_{12}$. Both accompanying lipomas are just rostral to the LDM stalk. Reused from Pang et al. ${ }^{25)}$ with permission from Springer Nature. LDM : limited dorsal myeloschisis. 

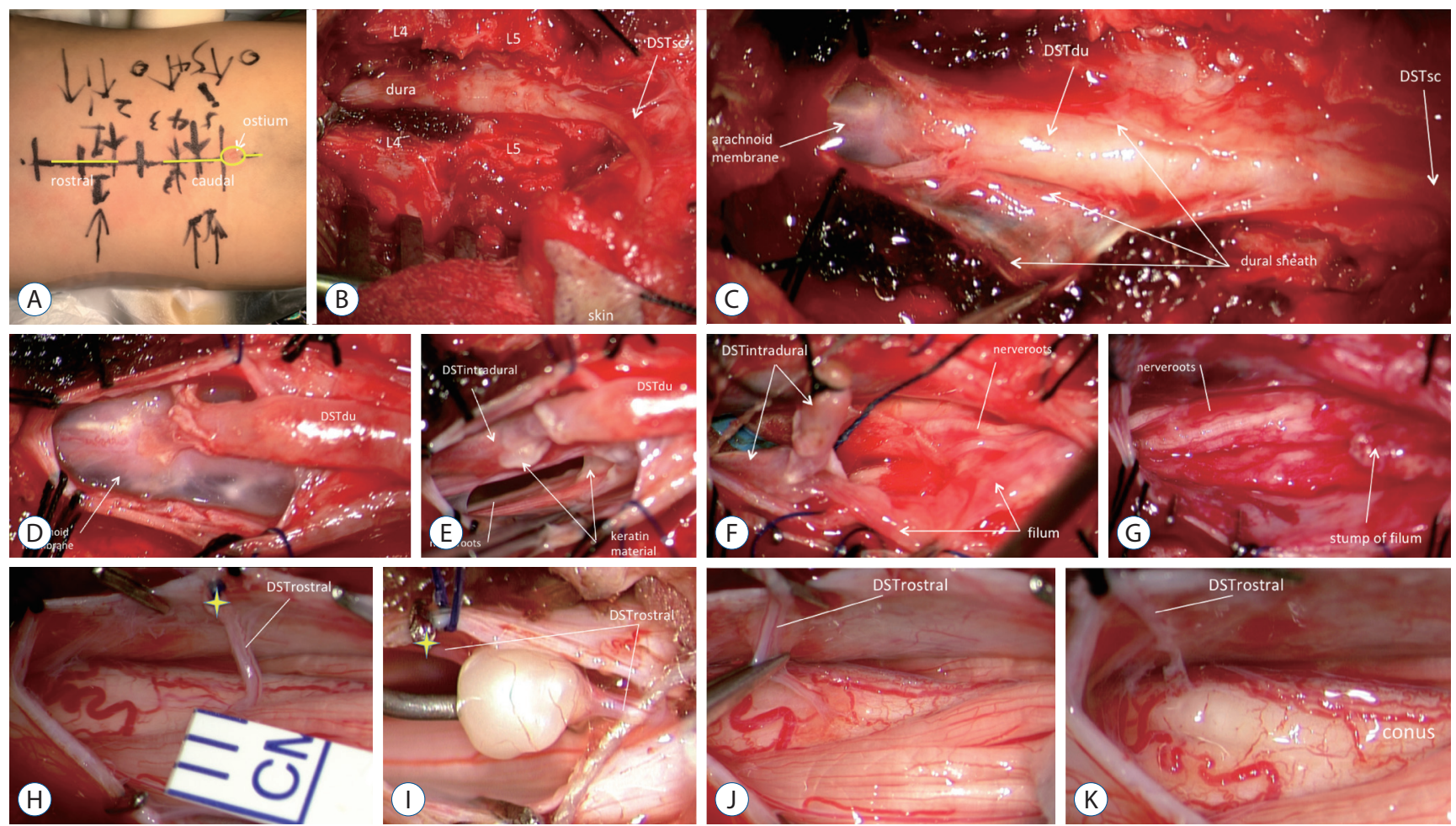

Fig. 47. Intra-operative photographs showing excision of a dermal sinus tract (DST) with skip laminectomy technique (MRI images of this patient are shown in Figs. 4 and 5). B-G : Caudal. H-K: Rostral wound. A : Skin preparation. Yellow lines mean skin incisions. Numbers in black on skin mean levels of lumbar spinous processes. B : L4L5 laminectomy has been done. The subcutaneous portion of the DST (DSTsc) merging with the dura has been fully exposed. C : Photography taken after opening the dural sheath enveloping a portion of DST that is lying in the dura mater (DSTdu). D : Photography showing the arachnoid membrane entry site of the DST. E : After opening the arachnoid membrane, the intradural portion of the DST (DSTintradural) and keratin material are seen. F : The DSTdu has been removed. The DSTintradural is seen adhering to the filum. G : The caudal portion of the DST has been completely removed via the L4L5 laminectomy. H and I : Operative exposure via a T12L1 laminectomy. (H) is the T12 side of the exposure; (I) the L1 side. A dermoid cyst along a slender DST (DSTrostral) is shown in (I). The DSTrotral was then cut at the yellow cross, and the cyst with the portion of DST under the intact L2 and L3 laminae delivered from this exposure. The sub-millimeter thickness of the DST testifies to the difficulty in detecting them with MRI. J and K : Complete removal of the deep end of the DST from the dorsal midline of the spinal cord. Reused from Wong et al. ${ }^{43}$ with permission from Springer Nature. MRI : magnetic resonance imaging.

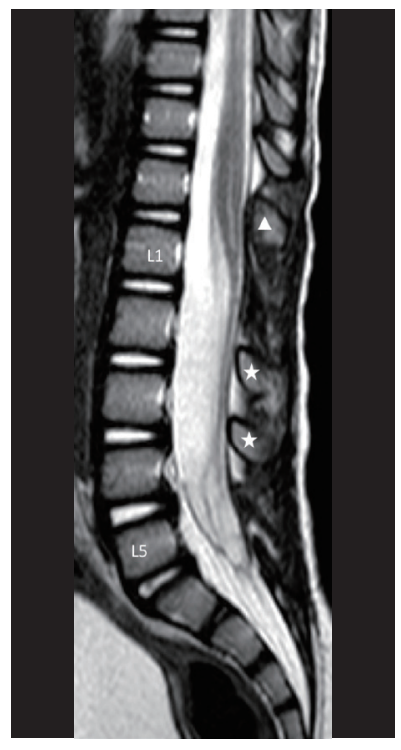

Fig. 48. Mid-sagittal MRI image showing the post-operative appearance of a skip laminectomy technique in which T12 laminoplasty, L1 laminectomy, and L4L5 laminectomy were done. White arrowhead means laminoplasty level. White stars mean intact laminae level. Reused from Wong et al. ${ }^{43}$ with permission from Springer Nature. significant adverse impact due to their inherent risks of inflammation, infection, proliferation producing mass effect, and recurrence if not totally excised. One should therefore always be vigilant for hidden dermal elements when dealing with spinal dysraphic malformations.

\section{EVALUATION}

A thorough clinical history and physical examination are paramount to elicit the symptoms and signs of neurological deficits related to the types and spinal level of FSNDs, past or active infection, and clues to the existence of associated anomalies. Urological assessment including urinalysis, ultrasonography of the urinary system, voiding cystourethrogram, and 

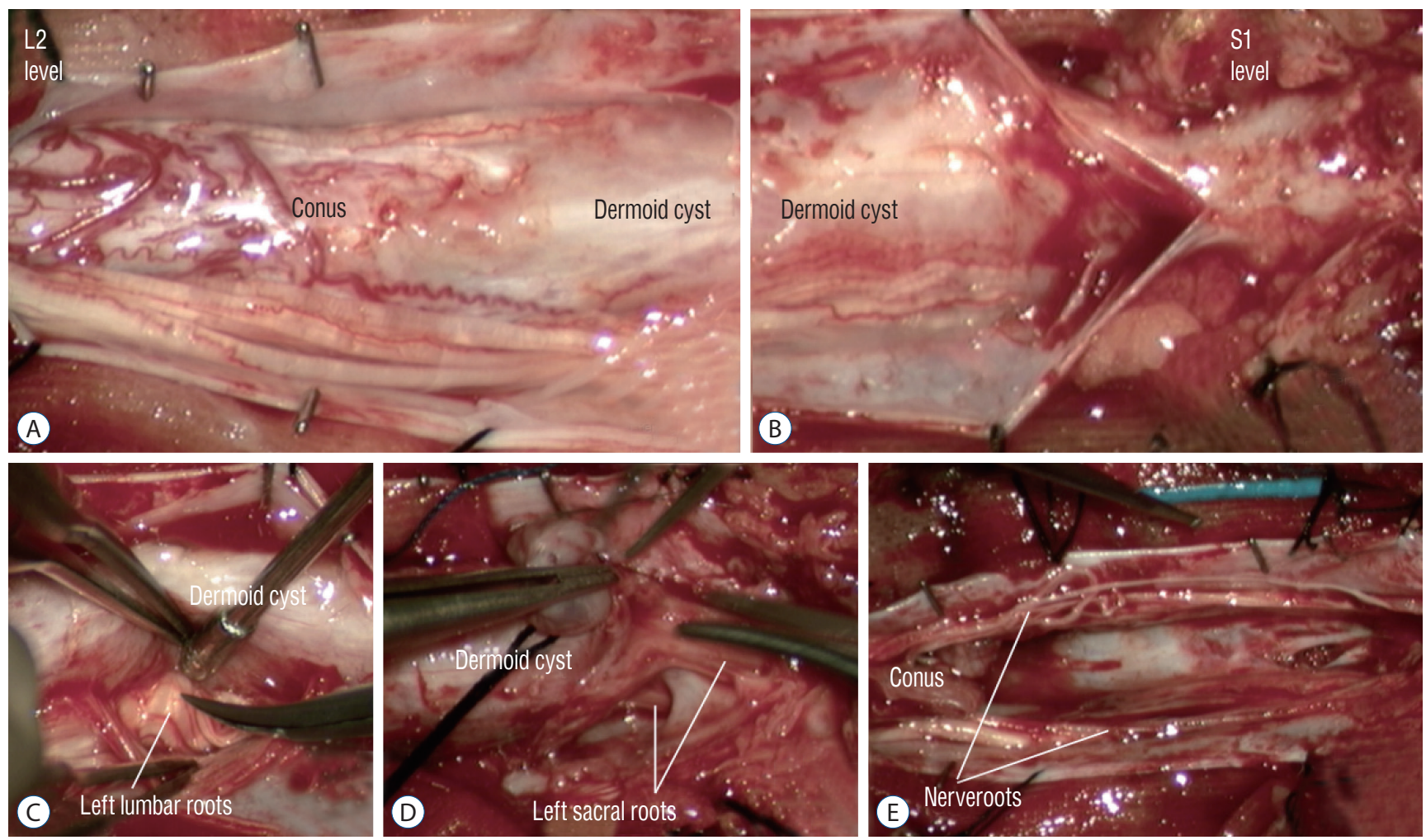

Fig. 49. Intra-operative photographs showing complete excision of an intradural dermoid cyst via L2-L5 laminoplasties and S1 laminectomy (MRI images are shown in Fig. 7). A and B : Surgical view after opening of dura. The dermoid cyst appears to merge with the conus and nerve roots. The dermoid cyst communicates with the skin ostium via a dermal sinus tract at the S1 laminar level. C and D : Dissection to free the nerve roots from the wall of the dermoid cyst. E : Excision cavity after complete excision of the dermoid cyst. Reused from Wong et al. ${ }^{43}$ with permission from Springer Nature. MRI : magnetic resonance imaging.

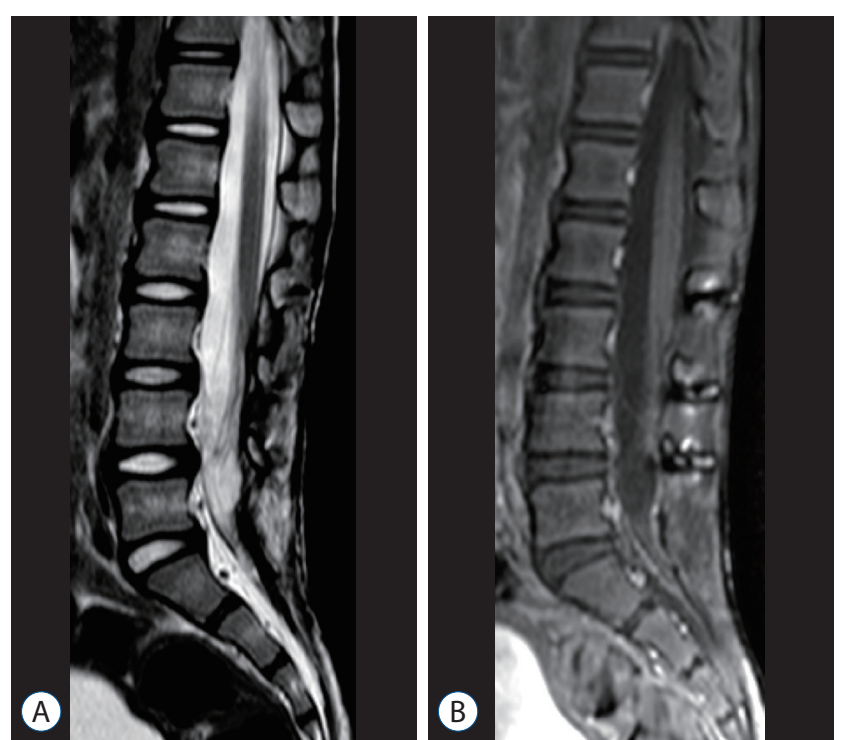

Fig. 50. Six-year post-operative MRI images (of the patient shown in Figs. 7 and 49) showing no recurrence of the dermoid cyst, and postlaminoplasty changes. A : T2-weighted MRI. B : T1-weighted MRI with gadolinium injection. Reused from Wong et al. ${ }^{43}$ ) with permission from Springer Nature. MRI : magnetic resonance imaging. also urodynamic studies should be performed.

MRI is the imaging technique of choice to delineate the details of pathological anatomy of the malformation especially regarding composite lesions. MRI findings suggestive of FSND are : 1) a tract linking the skin and the spinal cord even it does not appear continuous (Figs. 4-6, 10); 2) posteriorly tacked-up spinal cord (Figs. 10, 11, 13-15); and 3) a cystic lesion over the dorsal midline (Figs. 7, 12, 17, 21-23). When FSND is suspected on the MRI, the entire path of the tract must be traced from the skin through subcutaneous tissue, lamina, dura, and to the spinal cord. The constituents of the tract are interpreted as much as possible (Fig. 45) : any cyst along its course or in the vicinity is particularly noted (Figs. 7, 12, 17, 21-23), as is the presence of any associated anomalies especially spinal cord lipoma and split cord malformation. Lastly, the whole spinal axis should be surveyed for the rare coexistence of multiple FSNDs in the same spine (Fig. 46).

However, the reliability of MRI in delineating FSNDs is far from absolute. For example, a small tract can be below its res- 

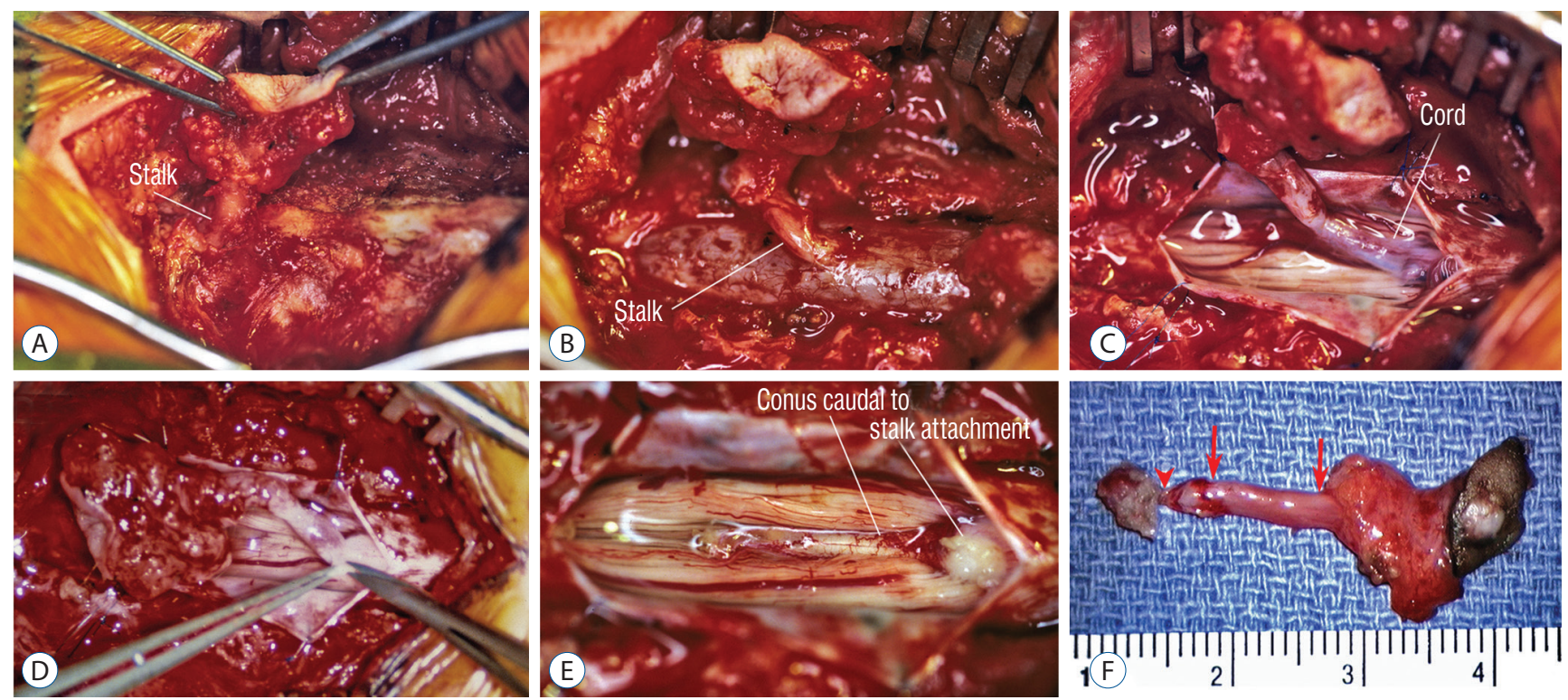

Fig. 51. Surgical resection of a lumbar crater-type flat (non-saccular) limited dorsal myeloschisis. A : Ellipse of resected skin crater and subcutaneous tract going through defect in lumbodorsal fascia. B : Extradural stalk and dural fistula. C: Intradural exposure showing stalk-cord union. D : Resection of stalk flush with cord surface. E : Normal conus caudal to stalk attachment site. F : En bloc specimen showing, from right to left, skin ellipse bearing pale epithelial crater, the subcutaneous portion bearing fat, the extradural portion of the stalk (between arrows), intradural stalk (between arrow and arrowhead), and an exuberant cuff of tissue on the cord. Reused from Pang et al. ${ }^{25)}$ with permission from Springer Nature.
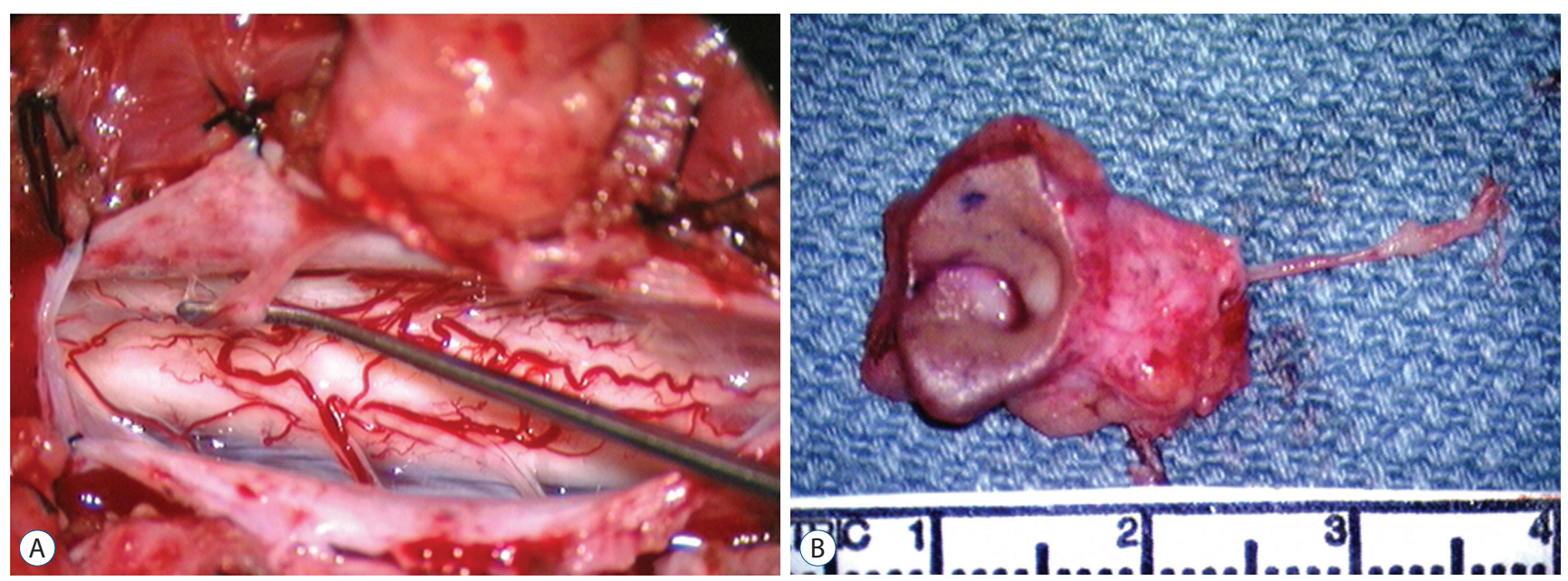

Fig. 52. Exceedingly slender limited dorsal myeloschisis (LDM) stalk. A : Stalk attaches to discrete spot on dorsal cord surface. B : En bloc specimen shows large complicated skin crater and the very slender LDM Stalk. Reused from Pang et al. ${ }^{25)}$ with permission from Springer Nature.

olution (Fig. 5) ${ }^{12,38)}$, and it cannot always differentiate a CSDST from a LDM, except when the CSDST becomes inflamed and exhibit abnormal enhancement of the tract, a constituent cyst, and adjacent meninges (Fig. 4) ${ }^{4}$. All are essential information for surgical planning.

\section{MANAGEMENT}

All FSNDs can cause functional impairment by tethering of the spinal cord, while CSDST or any of its mixed forms pose additional risks of inflammation, infection, mass effect, and even secondary hydrocephalus if left untreated. Early surgery should be performed in all patients with CSDST, and in symptomatic patients with LDM. In asymptomatic children 

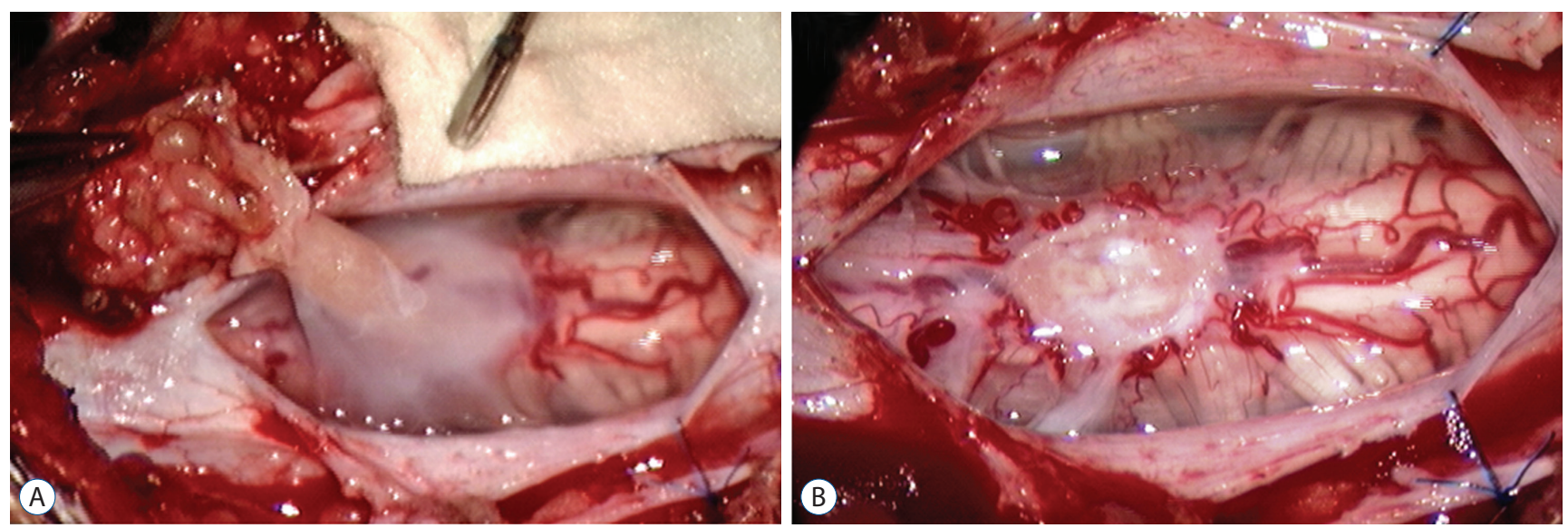

Fig. 53. Moderate-sized limited dorsal myeloschisis stalk. A : Stalk has a flared-out cord attachment. B : Stalk resection leaves a fish-mouth shaped scar. Reused from Pang et al. ${ }^{25)}$ with permission from Springer Nature.

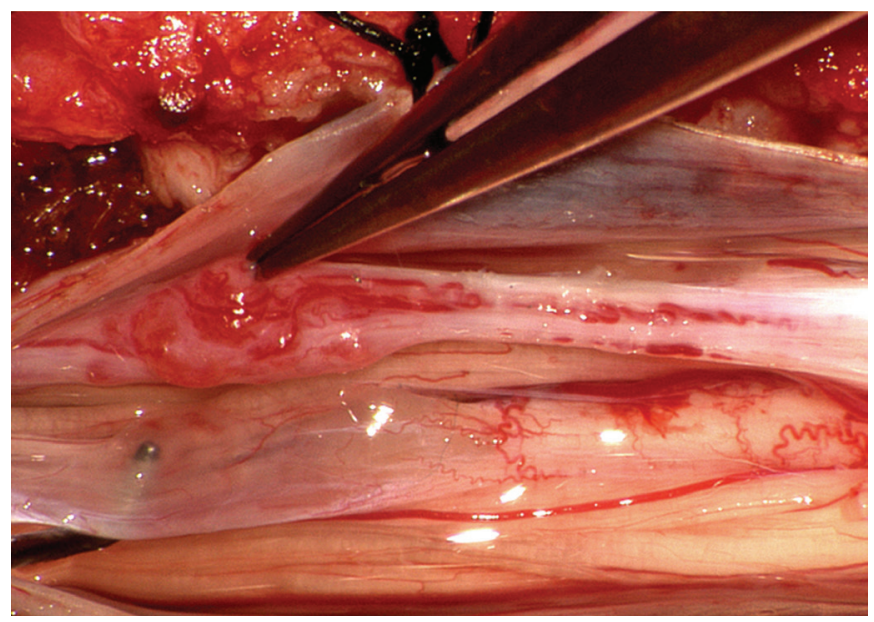

Fig. 54. Long limited dorsal myeloschisis stalk with vascular glomus (at tip of forceps). Reused from Pang et al. ${ }^{25)}$ with permission from Springer Nature.
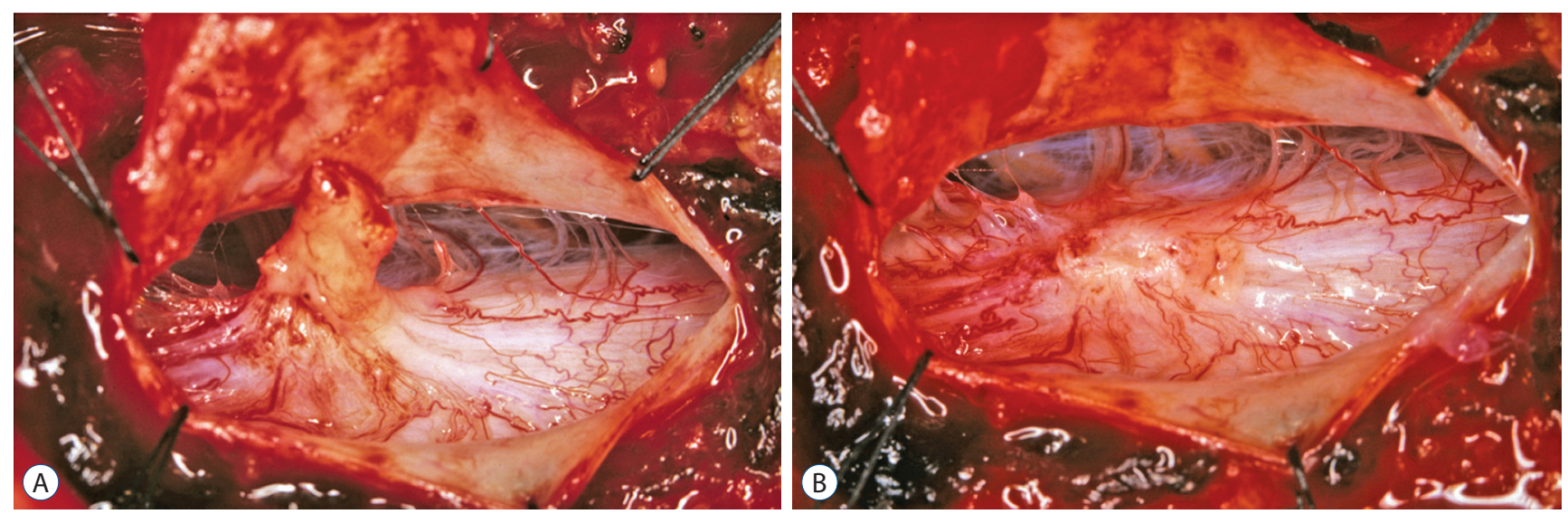

Fig. 55. Moderately thick limited dorsal myeloschisis (LDM) stalk with exuberant tentacles of blood vessels that seem to crawl on to the dorsal surface of the spinal cord. A : Before LDM resection. B : After LDM stalk resection showing centripetal distribution of the blood vessels. Reused from Pang et al. ${ }^{25}$ ) with permission from Springer Nature. 

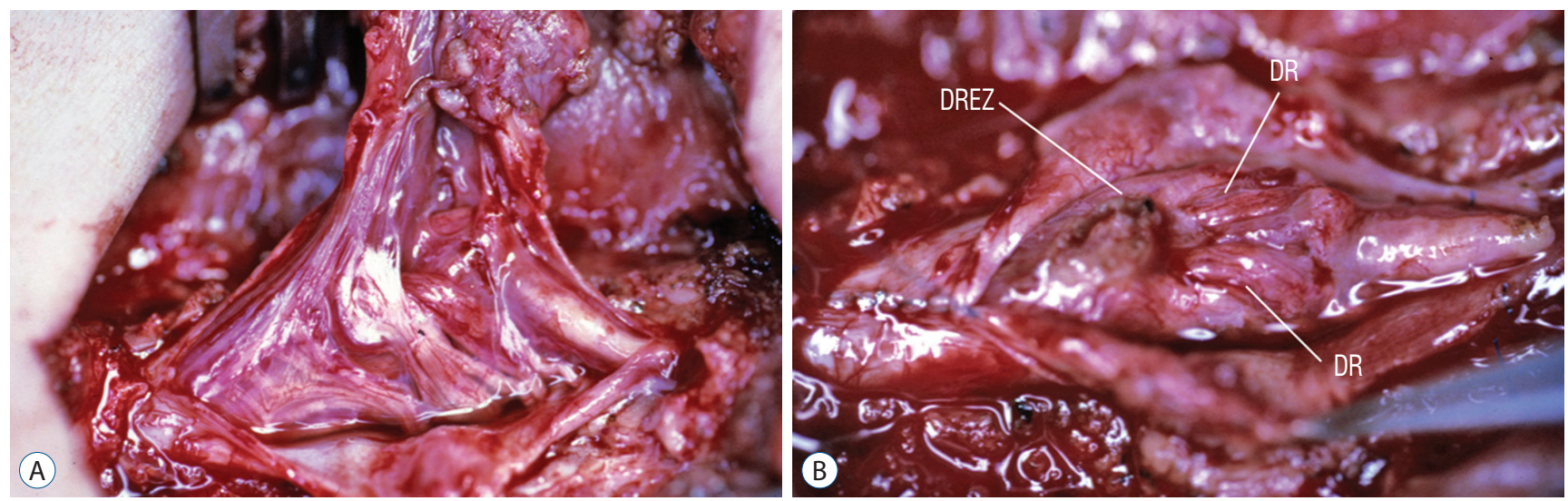

Fig. 56. Thick, complex-looking fibroneural stalk in a lumbar non-saccular LDM. A : Lesion contains dysplastic spinal cord tissue, large blood vessels, ample skein of non-functioning nerves, and thickened folded membranes. Spinal cord is lifted dorsally by the tethering effect. B : Bizarre arrangement of dorsal roots (DR), and dorsal root entry zone (DREZ) surround a large flat myeloschistic scar after stalk resection. Reused from Pang et al. ${ }^{25)}$ with permission from Springer Nature.
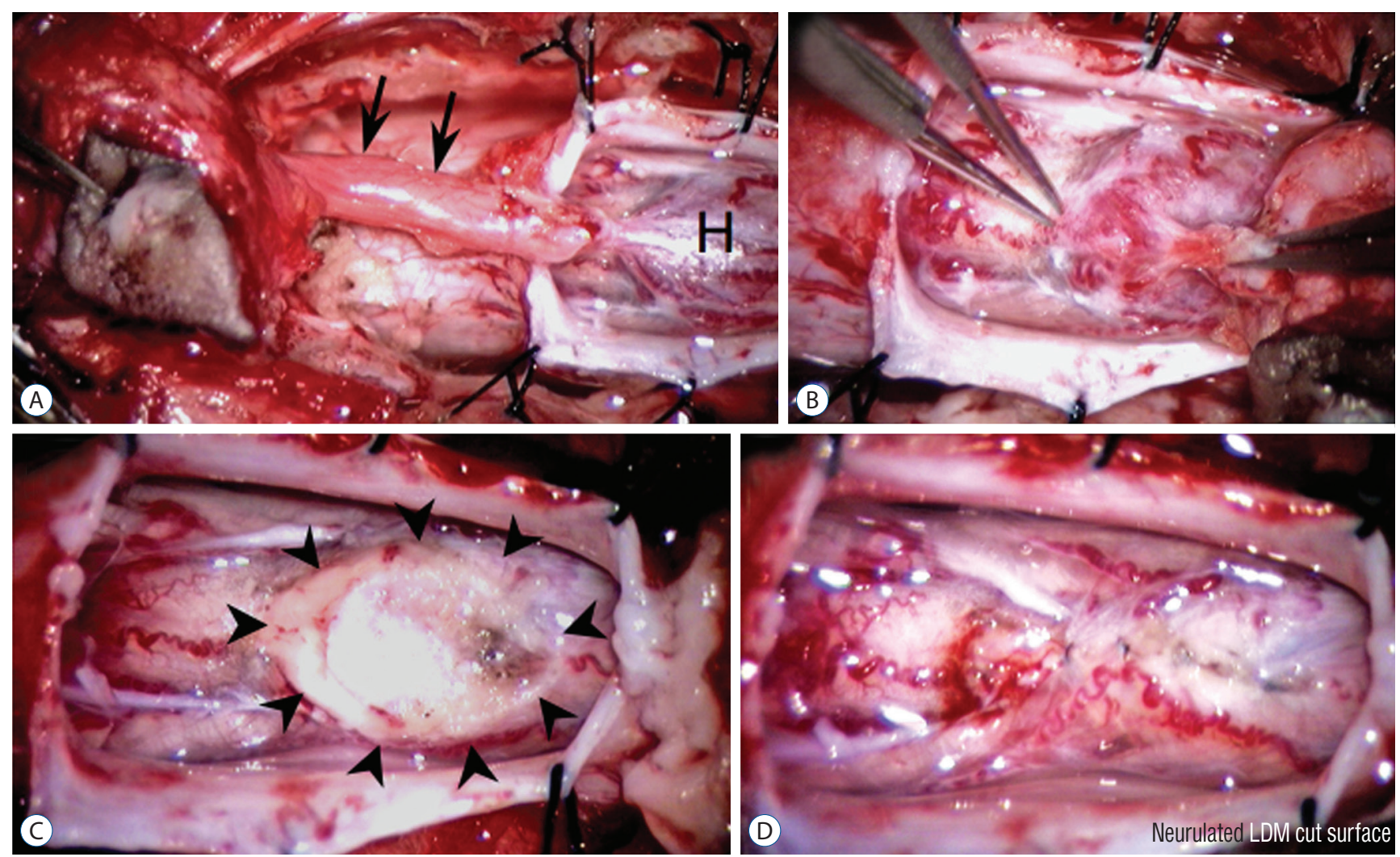

Fig. 57. Crater type lumbar limited dorsal myeloschisis (LDM). A : Lumbar LDM stalk (arrows) with a very prominent hump (H) of abnormal tissues on the cord. B : Resection at the base of this hump. C : Resection produced a very large scar (outlined by arrowheads). D : Dorsal pia-to-pia approximation of scar with 8-O Nylon sutures. Reused from Pang et al. ${ }^{25)}$ with permission from Springer Nature.

with pure LDM, we also strongly recommend surgery to obviate the dreadful consequences of late and unrecognized tethering. Observation by serial MRI is probably only suitable for patients with equivocal MRI findings or perhaps in asymp- tomatic adults.

For CSDST patients with active infection and neurological deficits, urgent surgery covered with appropriate antibiotics should be done. However, if infection is not accompanied by 

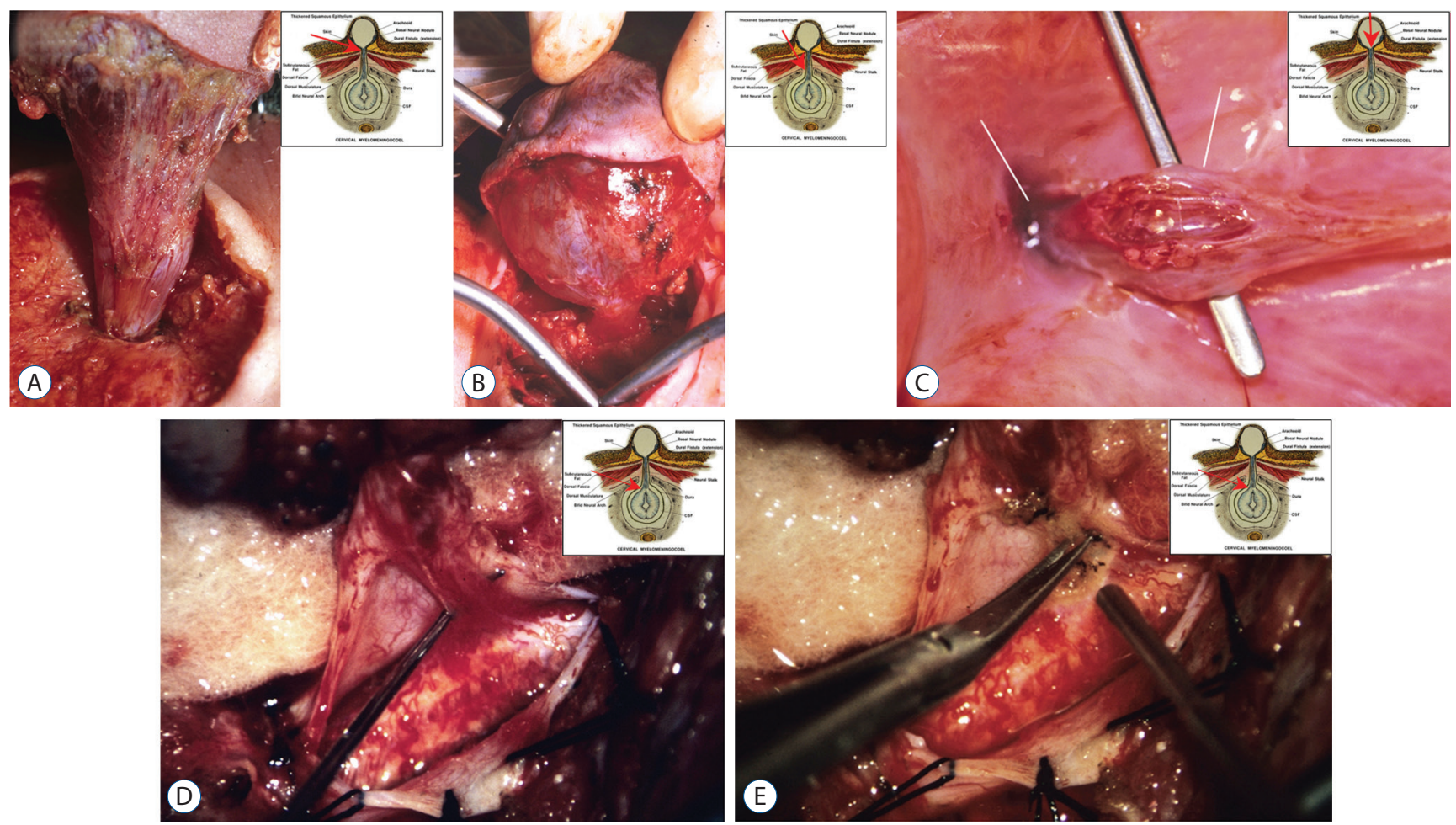

Fig. 58. Large cervical saccular limited dorsal myeloschisis. A : Exposure of the dorsal fistula at the level of the nuchal fascial defect. B : The neck of the sac passing through large laminar defect. C : The sac is opened from the top; basal neural nodule and the dural fistula opening (into the cyst) are seen through the cavity. D : Dural fistula opened into the main thecal sac, showing the thin fibroneural stalk inserting on to the dorsal spinal cord. E : Stalk being resected. All insets show exact level of the exposure. Reused from Pang et al. ${ }^{25)}$ with permission from Springer Nature.

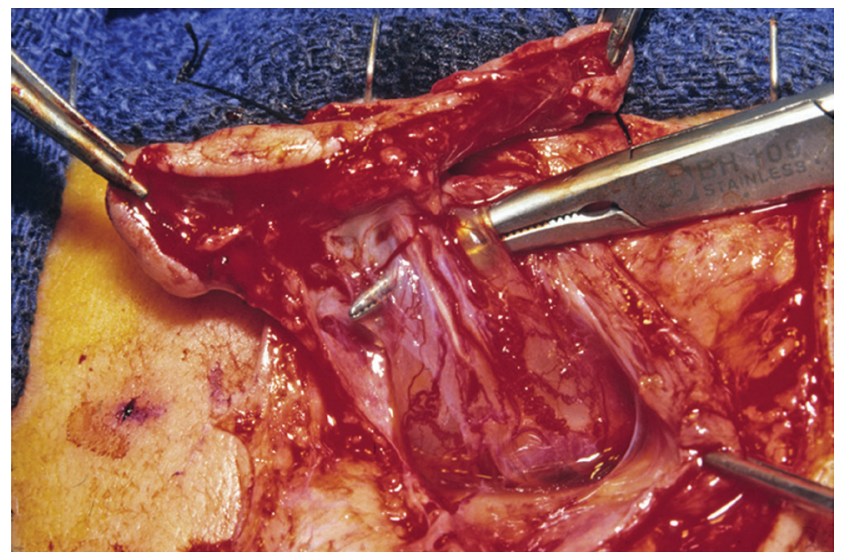

Fig. 59. Thoracic Saccular limited dorsal myeloschisis with the fibroneural stalk (displayed by instrument) traversing the sac cavity and reaching the dome of the sac. Reused from Pang et al. ${ }^{25)}$ with permission from Springer Nature.

neurological deficits, surgery should be deferred until the infection has been treated with antibiotics and local therapy.

\section{Surgery}

The principles of surgery in all FSNDs are to completely untether the spinal cord and to remove all epithelial elements if present. For pure forms, a narrow laminectomy for exposure is usually adequate. Laminoplasty is an option, and dural grafting is rarely necessary. In cases with a large intradural dermoid cyst, however, wider bony exposure is usually needed. The extent of longitudinal exposure must include the span between the skin lesion and where the tract joins the spinal cord, which is usually apparent where the cord outline suddenly becomes trapezoid instead of the normal ovoid. In uncertain cases, the skin should be widely draped to accommodate for extension of the incision. If the lesion is in the lumbosacral region, the filum terminale may be thickened and should be cut during treatment of the FSND, so that appropriate provision must be made for more caudal exposure.

\section{Surgical technique for CSDST}

The patient is laid prone, the sinus ostium identified, and the laminae of the planned laminectomy confirmed with ra- 

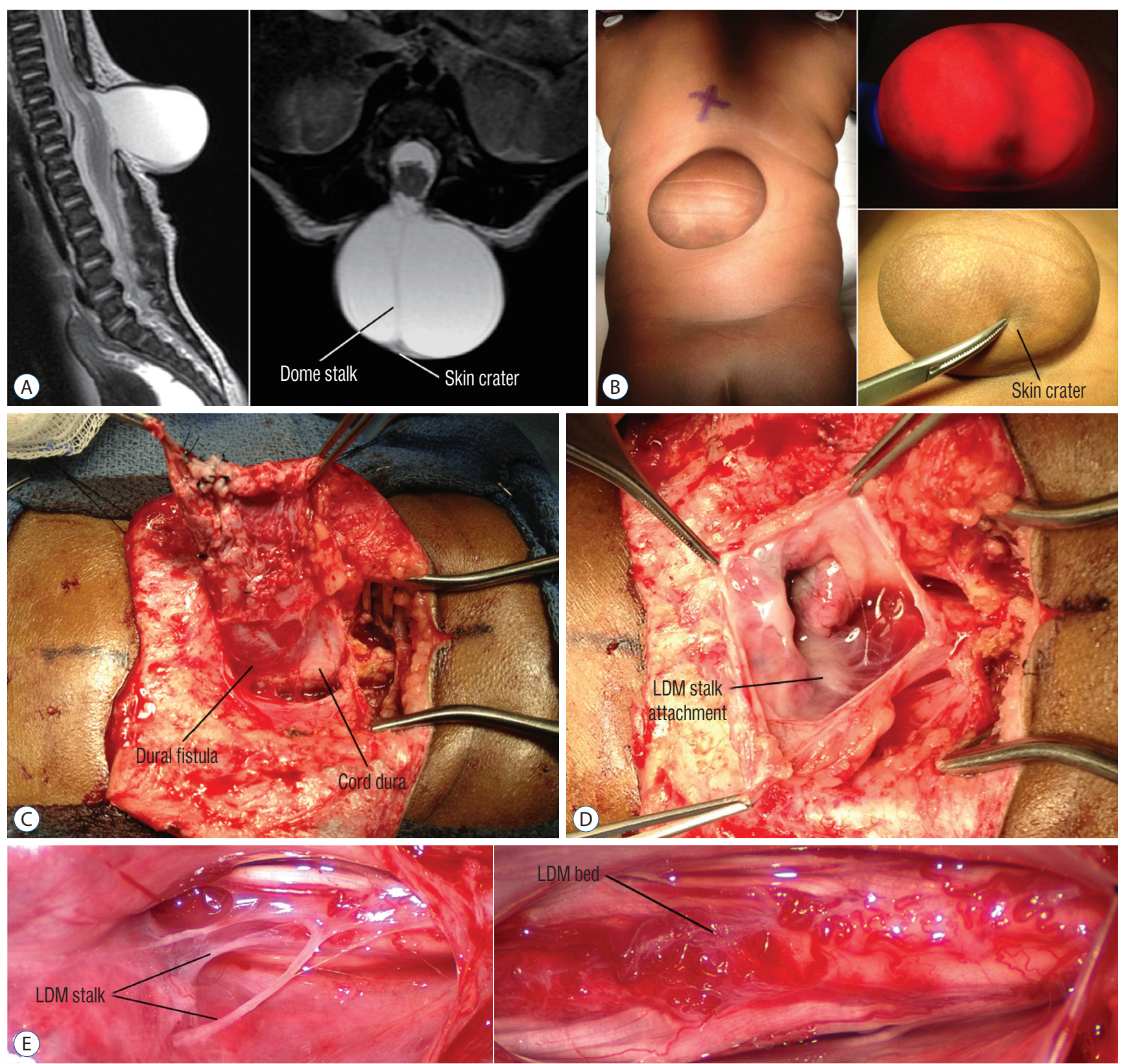

Fig. 60. Saccular limited dorsal myeloschisis (LDM) with the stalk-to-dome subtype of fibroneural stalk attachment. $A: T_{2}$ magnetic resonance imaging shows slight tenting of the cord towards the sac on the sagittal image, and the fibroneural stalk (dome stalk) traversing the sac to the cord from the base of the skin crater in the axial image. B : The sac with the slightly darker irregular skin at the lower dome with a slightly thinner covering (skin crater, lower right) that may be thick squamous epithelium. The transilluminated picture (upper right) shows the small nubbin of (neural) tissue beneath the skin crater, and the stream of bands traversing the middle of the sac. C : Shows the wide neck of the dural fistula at the base of the sac, and its relationship with the cord dura. D : Sac opened from the top, showing the white area where the LDM stalk attaches to the cord surface. $E$ : Close-up to show the strands of the LDM stalk inserting on the dorsal cord surface (upper). After resecting these strands (lower), the cord surface shows abnormal clusters of wiggly blood vessels and scar tissue. Reused from Pang et al. ${ }^{25)}$ with permission from Springer Nature.

diograph (Fig. 47). Standard midline longitudinal skin incision with a small elliptical island around the ostium is made; the tract is then traced from superficial to deep through the subcutaneous layers and deep fascia, to reach the bifid spinous process or through the interspinous ligament. The laminectomy is then carried out carefully around the tract. Dissection of the tract should be done under magnification to minimize the possibility of leaving behind residuum ${ }^{4}$. The dura should 

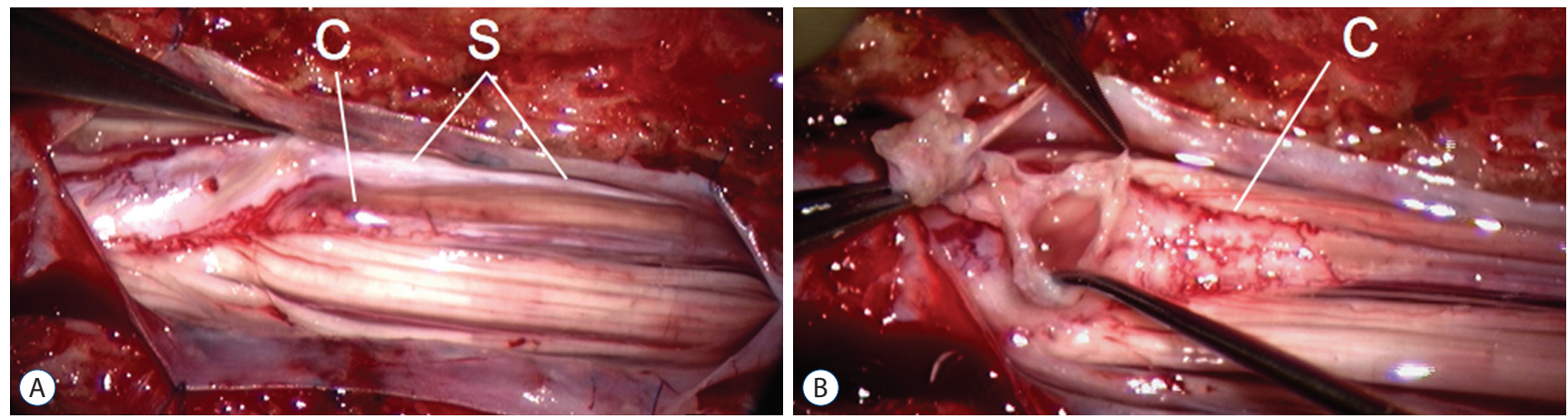

Fig. 61. Lumbar saccular limited dorsal myeloschisis with segmental myelocystocoele. A : A long intradural stalk (S) picked up by micro-forceps. B : Stalk traced to the hydromyelic portion of the cord after partial stalk resection. Reused from Pang et al ${ }^{25}$ ) with permission from Springer Nature. C : conus.
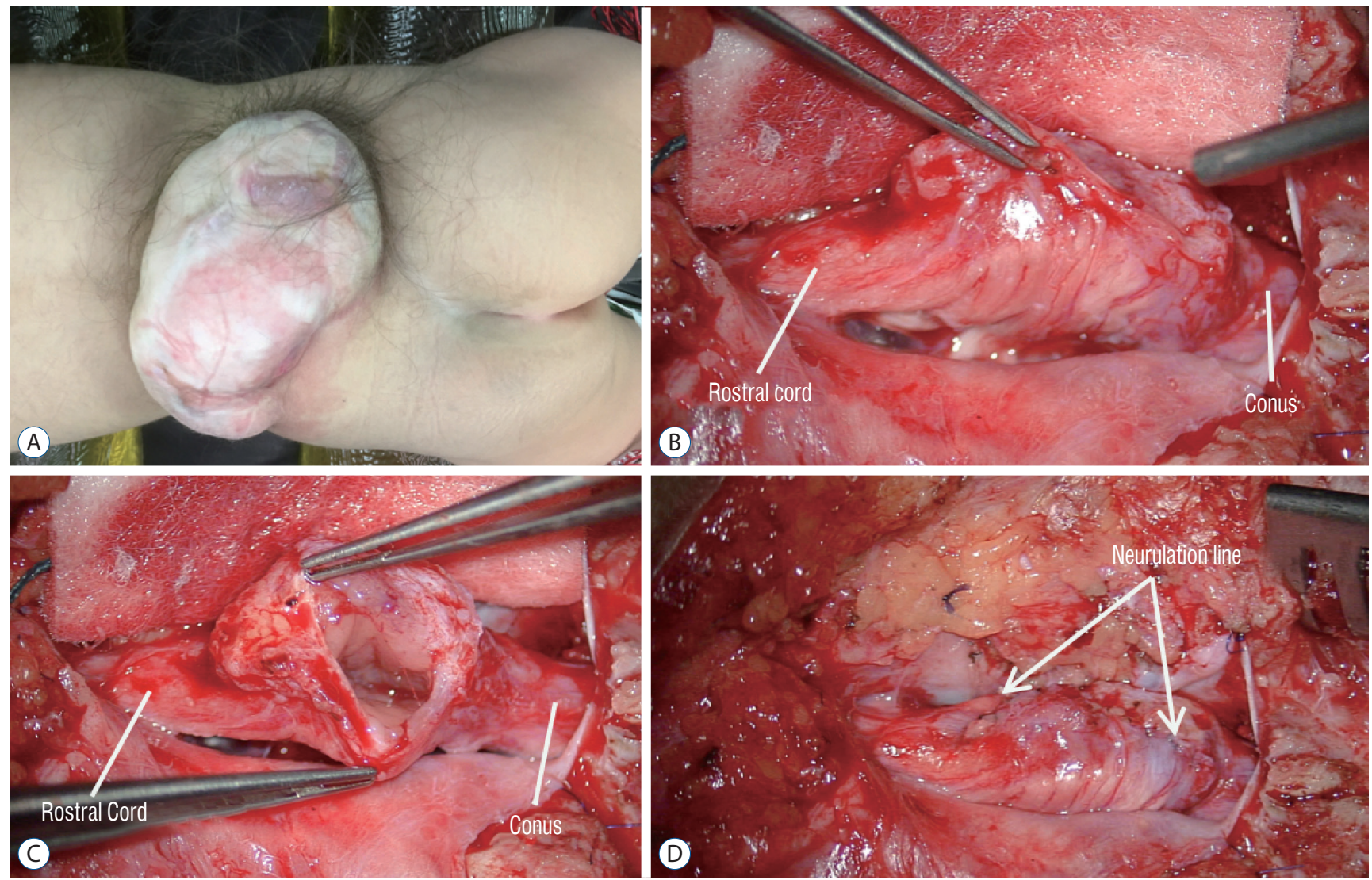

Fig. 62. Intraoperative photos of the case shown in Fig. 19. A : A large skin-based sac with pearly epithelium, and hypertrichosis around the base of the sac. B : The non-functional top of the cystocoele has been cut off leaving the functional base with nerve roots. C: Operative view looking through the neck of the cystocoele into the hydromyelic cavity. D : After neurulation closure of the myelocystocoele neck.

always be opened unless the surgeon is absolutely certain that the tract ends outside the dura. When the tract goes intradurally, a cuff of dura may need to be excised with the tract, and the latter should be traced to its destination spot on the spinal cord. Often the tract becomes attenuated and loosely perches on the surface of the cord. Adequate bony exposure must be done without compromise to display the full extent of the tract. If a long tract truly spans many laminar levels, skip laminectomy technique should be considered in which some laminae between the tract's dural entry point and its spinal cord attachment point are strategically kept intact. The CSDST tract can be carefully delivered from the laminectomy wounds 

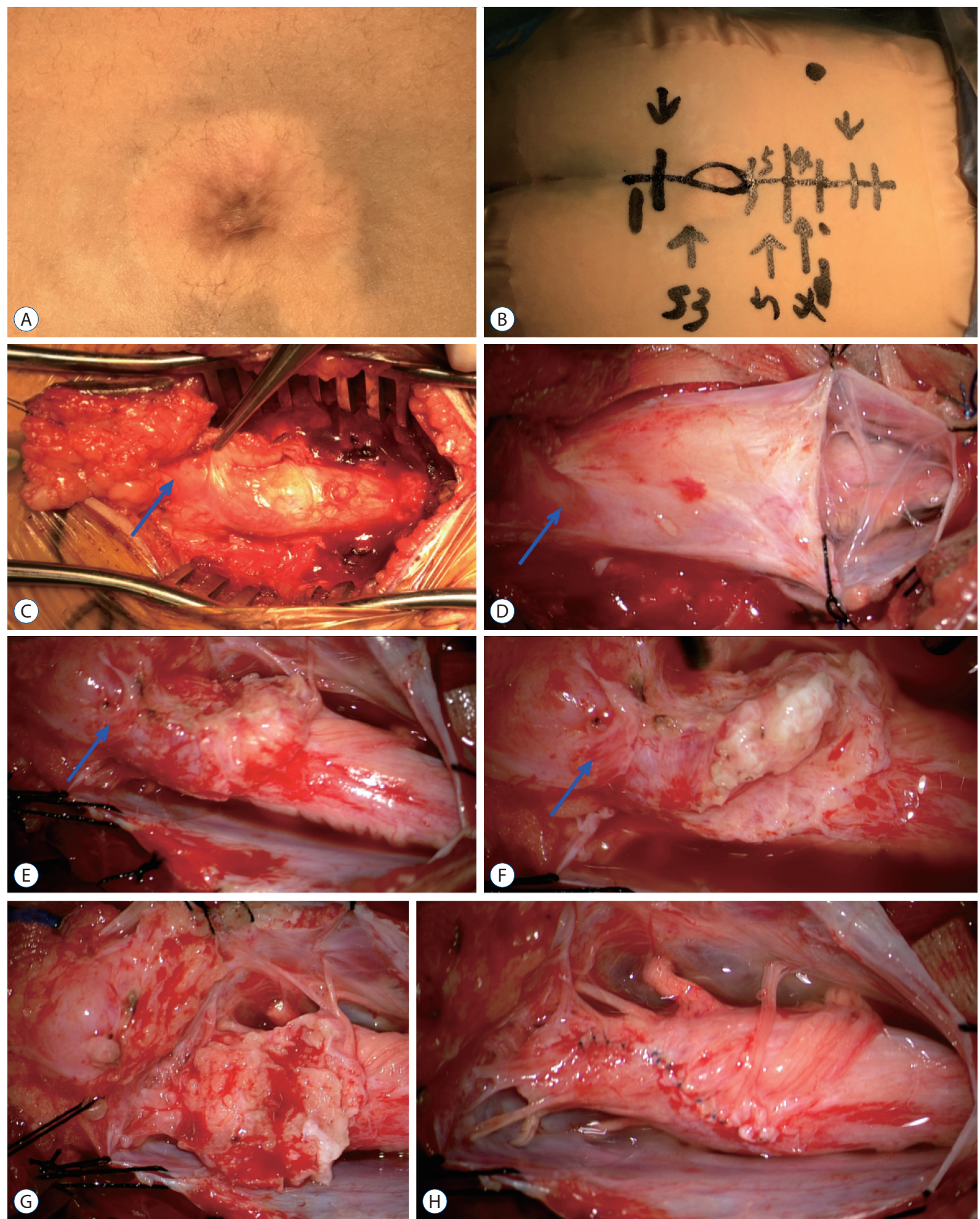

Fig. 63. Intra-operative photographs showing the excision of a LDM - lipoma complex (MRI images are shown in Fig. 45). A : The skin lesion, a cigar burn crater with surrounding skin discoloration. B: Markings for the skin incision. C : Surgical exposure after L4-S2 laminectomies. D : The dura has been opened. E : Surgical exposure after completion of "crotch dissection". F : Detaching the stout LDM stalk and lipoma from the spinal cord. G : Residual fat on the placode before final trimming. $\mathrm{H}$ : Appearance of the spinal cord after neurulation. The nerve roots at the end of the spinal cord were stimulation positive. Blue arrow means Extradural portion of the LDM. The patient has no neurological deficits before and after the untethering surgery. Reused from Wong et al. ${ }^{43)}$ with permission from Springer Nature. LDM : limited dorsal myeloschisis, MRI : magnetic resonance imaging. 

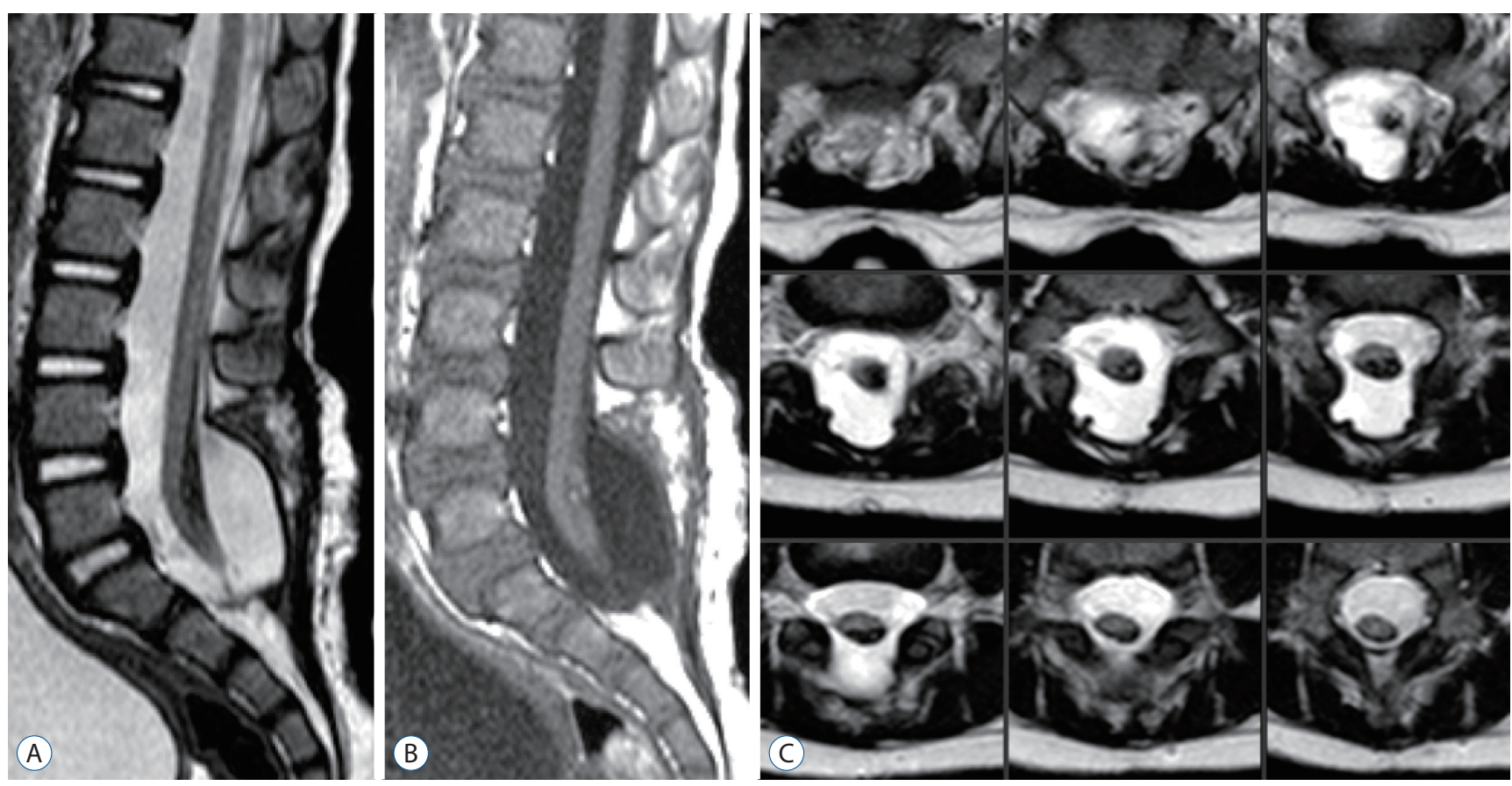

Fig. 64. Post-operative MRI images of the patient shown in Fig. 63. A : T2-weighed sagittal MRI image showing a cord-sac ratio of 33\%. B :T1-weighted sagittal MRI image. C : Serial T2-weighted axial MRI images showing the spinal cord completely surrounded by cerebrospinal fluid. Reused from Wong et $a{ }^{43}{ }^{43}$ with permission from Springer Nature. MRI : magnetic resonance imaging.

Table 4. Pre-operative, 3-month post-operative, and 1 year post-operative neurological grades in LDM* patients grouped against LDM location

\begin{tabular}{|c|c|c|c|c|c|c|c|c|c|c|c|c|}
\hline \multirow{3}{*}{ LDM location } & \multirow{2}{*}{\multicolumn{4}{|c|}{ Pre-operative grade }} & \multicolumn{8}{|c|}{ Post-operative grade } \\
\hline & & & & & \multicolumn{4}{|c|}{3 months } & \multicolumn{4}{|c|}{1 year } \\
\hline & 0 & 1 & 2 & 3 & 0 & 1 & 2 & 3 & 0 & 1 & 2 & 3 \\
\hline Cervical $(n=11)$ & 4 & 4 & 3 & 0 & 4 & 5 & 2 & 0 & 7 & 4 & 0 & 0 \\
\hline Thoracic upper $(n=9)$ & 2 & 6 & 1 & 0 & 3 & 6 & 0 & 0 & 6 & 3 & 0 & 0 \\
\hline Thoracic lower $(n=7)$ & 4 & 2 & 0 & 1 & 4 & 2 & 1 & 0 & 4 & 2 & 1 & 0 \\
\hline Thoracolumbar $(\mathrm{n}=8)$ & 4 & 3 & 0 & 1 & 4 & 3 & 1 & 0 & 5 & 3 & 0 & 0 \\
\hline Lumbar (n=28) & 15 & 8 & 3 & 2 & 17 & 6 & 4 & 1 & 20 & 7 & 0 & 1 \\
\hline Total number of patients ( $n=63$ ) & 29 & 23 & 7 & 4 & 31 & 22 & 8 & 1 & 41 & 19 & 1 & 1 \\
\hline
\end{tabular}

Reused from Pang et al. ${ }^{25}$ ) with permission from Springer Nature. *Neurological grading system in LDM : grade 0, no deficits or symptomsk; grade 1 , mild upper or lower extremity weakness, or pure sensory deficits \pm pain; grade 2, moderate to severe upper or lower extremity weakness \pm sensory deficits, or neurogenic bladder without weakness; grade 3, upper or lower extremity weakness+neurogenic bladder. LDM : limited dorsal myeloschisis

(Fig. 48). Afterwards, primary closure of the dura is usually possible.

The sinus tract may expand along its course or terminate in a dermoid or epidermoid cyst. Extradurally located cysts are readily excised. For large intradural cysts, refined microsurgical techniques are required for their complete removal since the cyst wall is notoriously adherent to nerve roots and pia (Figs. 49 and 50). Microbial cultures from adjacent areas should be obtained, and post-operative antibiotics should be given until negative growth is confirmed.

\section{Surgical technique for flat LDM}

The surgical strategy for flat LDMs is similar to that for CSDST. After a standard midline skin incision, the skin crater or pit is excised and the stalk at the base of the skin lesion is carefully dissected out and traced through the defects in myofascial layers, and laminae or interspinous ligament (Fig. 51A). To provide good exposure of the stalk-spinal cord attachment for 
a safe excision, at least one level of laminectomy both rostral and caudal to the stalk-spinal cord merge point should be planned (Fig. 51B). Again, if the stalk spans multiple levels, skip laminectomy may be considered.

A midline durotomy is made, centred upon the entry point of the fibroneural stalk and extended according to the track of the stalk as demonstrated on the MRI. The usually slender stalk is most often attached to a discrete linear spot or cleft on the dorsal midline of the cord (Fig. 51C); it is simply cut flush with the cord surface (Fig. 51D and E). Any peripheral nerve twigs, blood vessels, and fibrous bands encircling the neural stalk are similarly cut. Once the intradural stalk had been disconnected from the cord, the entire stalk with its skin appendage is resected en bloc (Fig. 51F).

The LDM stalk may be exceedingly slender and attaches to the cord in a minute midline scar (Fig. 52), or the stalk flares out into a wider hold on the cord so that the cut edge on the cord resembles a gaping fish mouth (Fig. 53). The stalk may also contain a glomus of vascular channels (Fig. 54), or its deep end expands into tentacles of blood vessels that crawl on the cord (Fig. 55). Rarely, the stalk attachment is stout and deceptively complex, and the dorsal roots surround it like a cuff (Fig. 56). These attachments are cut flush as above but sparing the surrounding nerve root to reveal a large base of raw spinal cord (Fig. 56B). Rarely, it is necessary to approximate the pial edges of the large raw bed of a pure LDM to eliminate a potentially adherent surface susceptible to re-tethering (Fig. 57). The dura is closed primarily.

\section{Surgical technique for saccular LDMs}

The surgical technique for tackling the internal structures of LDMs is basically the same whether the LDM is saccular or flat. The minor differences between handling the two types lay in the initial superficial soft tissue dissection. For the saccular LDMs, a large skin ellipse is made at the sessile base of the sac to expose the dural funnel where the narrow dural fistula fans out to form the sac at the skin level (Fig. 58A). The dural fistula is then traced to the lamina level as with the flat LDM (Fig. 58B).

For the basal nodule type of saccular LDM, the sac is entered at the base to locate the basal neural nodule (Fig. 58C) and the underlying fibroneural stalk within the dural fistula, where it is traced to its attachment to the cord and removed (Fig. 58D and E). Saccular LDMs with very thick stalks that traverse the sac to reach the dome are exposed from the spinal cord side up towards the top (Fig. 59). The base of the stalk is then disconnected from the cord surface. In large saccular lesions with slender stalks that may be hard to find, the sac is opened at the dome and the fibroneural stalk is located at the base of the abnormal skin crater, then traced to the spinal cord surface where it is transected (Fig. 60). In saccular LDM with myelocystocoeles, the stalk may be longer than expected and its cut end can often be traced directly into the hydromyelic cavity of the cord (Figs. 61 and 62).

\section{Surgical techniques for FSNDs associated with other anomalies}

The operative strategy for these conditions is a combination of the above techniques for FSNDs with the specific techniques suitable for the associated malformations ${ }^{25,26)}$. The technique for the associated malformation usually predominates (Figs. 63 and 64). The most salient point to note is the essential total extirpation of any dermal elements in a complex lesion.

\section{OUTCOMES AND CONCLUSION}

The clinical outcome of FSNDs is dictated by the presentation clinical status. With proper surgical techniques, surgery for FSNDs without large intradural dermoid/epidermoid cysts is usually uncomplicated; recurrence of dermal elements and re-tethering should be extremely rare; and most FSND patients without neurological deficits remain neurologically intact after surgery ${ }^{1,25,42)}$. Over two thirds of patients with preoperative neurological deficits improve after surgery; about one third will not improve though remain stable (Table $4)^{1,25,41)}$. In CSDST patients with large intradural dermoid/epidermoid cysts or active infections, the results are less salubrious ${ }^{1,7,40,43)}$. These statistics thus highlight the importance of early detection and prompt surgical intervention in FSNDs.

\section{CONFLICTS OF INTEREST}

No potential conflict of interest relevant to this article was reported. 


\section{INFORMED CONSENT}

This type of study does not require informed consent.

\section{AUTHOR CONTRIBUTIONS}

\author{
Conceptualization : DP, STW \\ Data curation : DP, STW \\ Formal analysis : STW \\ Funding acquisition : DP \\ Methodology : DP, STW \\ Project administration : STW \\ Visualization : STW \\ Writing - original draft : STW \\ Writing - review \& editing: DP
}

\section{ORCID}

$\begin{array}{ll}\text { Sui-To Wong } & \text { https://orcid.org/0000-0001-7940-8866 } \\ \text { Dachling Pang } & \text { https://orcid.org/0000-0002-6603-6546 }\end{array}$

\section{References}

1. Ackerman $L L$, Menezes AH : Spinal congenital dermal sinuses: a 30-year experience. Pediatrics 112(3 Pt 1) : 641-647, 2003

2. Colas JF, Schoenwolf GC : Towards a cellular and molecular understanding of neurulation. Dev Dyn 221 : 117-145, 2001

3. Cornips EM, Weber JW, Vles JS, van Aalst J : Pseudo-dermal sinus tract or spinal dermal-sinus-like stalk? Childs Nerv Syst 27 : 1189-1193, 2011

4. De Vloo P, Lagae L, Sciot R, Demaerel P, van Loon J, Van Calenbergh F : Spinal dermal sinuses and dermal sinus-like stalks analysis of 14 cases with suggestions for embryologic mechanisms resulting in dermal sinuslike stalks. Eur J Paediatr Neurol 17 : 575-584, 2013

5. Eibach S, Moes G, Zovickian J, Pang D : Limited dorsal myeloschisis associated with dermoid elements. Childs Nerv Syst 33 : 55-67, 2017

6. Elton $S$, Oakes WJ : Dermal sinus tracts of the spine. Neurosurg Focus $10: e 4,2001$

7. Girishan $S$, Rajshekhar $V$ : Rapid-onset paraparesis and quadriparesis in patients with intramedullary spinal dermoid cysts: report of 10 cases. J Neurosurg Pediatr $17:$ 86-93, 2016

8. Hiraoka A, Morioka T, Murakami N, Suzuki SO, Mizoguchi M : Limited dorsal myeloschisis with no extradural stalk linking to a flat skin lesion: a case report. Childs Nerv Syst 34 : 2497-2501, 2018
9. Kim JW, Wang KC, Chong S, Kim SK, Lee JY : Limited dorsal myeloschisis: reconsideration of its embryological origin. Neurosurgery 86 : 93100,2020

10. Lee JY, Chong S, Choi YH, Phi JH, Cheon JE, Kim SK, et al. : Modification of surgical procedure for "probable" limited dorsal myeloschisis. J Neurosurg Pediatr 19 : 616-619, 2017

11. Lee JY, Park SH, Chong S, Phi JH, Kim SK, Cho BK, et al. : Congenital dermal sinus and limited dorsal myeloschisis:"spectrum disorders" of incomplete dysjuction between cutaneous and neural ectoderms. Neurosurgery $84: 428-434,2019$

12. Lee SM, Cheon JE, Choi YH, Kim IO, Kim WS, Cho HH, et al. : Limited dorsal myeloschisis and congenital dermal sinus: comparison of clinical and MR imaging features. AJNR Am J Neuroradiol 38 : 176-182, 2017

13. Martínez-Lage JF, Almagro MJ, Ferri-Ñiguez B, Izura Azanza V, Serrano C, Domenech E : Spinal dermal sinus and pseudo-dermal sinus tracts: two different entities. Childs Nerv Syst 27 : 609-616, 2011

14. Martins-Green $\mathrm{M}$ : Origin of the dorsal surface of the neural tube by progressive delamination of epidermal ectoderm and neuroepithelium: implications for neurulation and neural tube defects. Development 103 : 687-706, 1988

15. Martins-Green M, Erickson CA : Basal lamina is not a barrier to neural crest cell emigration: documentation by TEM and by immunofluorescent and immunogold labelling. Development 101 : 517-533, 1987

16. Morioka T, Suzuki SO, Murakami N, Mukae N, Shimogawa T, Haruyama $\mathrm{H}$, et al. : Surgical histopathology of limited dorsal myeloschisis with flat skin lesion. Childs Nerv Syst 35 : 119-128, 2019

17. Morioka T, Suzuki SO, Murakami N, Shimogawa T, Mukae N, Inoha S, et al. : Neurosurgical pathology of limited dorsal myeloschisis. Childs Nerv Syst 34 : 293-303, 2018

18. Müller F, O'Rahilly $R$ : The prechordal plate, the rostral end of the notochord and nearby median features in staged human embryos. Cells Tissues Organs $173: 1-20,2003$

19. Müller F, O'Rahilly R : The primitive streak, the caudal eminence and related structures in staged human embryos. Cells Tissues Organs 177 : 2-20, 2004

20. Nikolopoulou E, Galea GL, Rolo A, Greene ND, Copp AJ : Neural tube closure: cellular, molecular and biomechanical mechanisms. Development 144 : 552-566, 2017

21. Pang $D$ : Surgical management of spinal dysraphism in Fessler $R$, Sekhar $L$ (eds) : Atlas of Neurosurgical Techniques. New York : Thieme Medical and Scientific Publishers, 2006, pp729-758

22. Pang D, Dias MS : Cervical myelomeningoceles. Neurosurgery 33 : 363-373, 1993

23. Pang D, Dias MS, Ahab-Barmada M : Split cord malformation part I: a unified theory of embryogenesis for double spinal cord malformations. Neurosurgery $31: 451-480,1992$

24. Pang D, Zovickian J, Oviedo A, Moes GS : Limited dorsal myeloschisis: a distinctive clinicopathological entity. Neurosurgery 67 : 1555-1580, 2010

25. Pang D, Zovickian J, Wong ST, Hou YJ, Moes GS : Limited dorsal myelos- 
chisis: a not-so-rare form of primary neurulation defect. Childs Nerv Syst 29 : 1459-1484, 2013

26. Pang D, Zovickian J, Wong ST, Hou YJ, Moes GS : Surgical treatment of complex spinal cord lipomas. Childs Nerv Syst 29 : 1485-1513, 2013

27. Quon JL, Grant GA : Commentary: limited dorsal myeloschisis: reconsideration of its embryological origin. Neurosurgery 86 : E13-E14, 2020

28. Rolo A, Escuin $\mathrm{S}$, Greene NDE, Copp AJ : Rho GTPases in mammalian spinal neural tube closure. Small GTPases 9 : 283-289, 2018

29. Rossi A, Piatelli G, Gandolfo C, Pavanello M, Hoffmann C, Van Goethem JW, et al. : Spectrum of nonterminal myelocystoceles. Neurosurgery $58:$ 509-515, 2006

30. Schoenwolf GC: Observations on closure of the neuropores in the chick embryo. Am J Anat 155 : 445-465, 1979

31. Schoenwolf GC, Smith JL : Mechanisms of neurulation: traditional viewpoint and recent advances. Development 109 : 243-270, 1990

32. Selleck MA, Bronner-Fraser $M:$ Origins of the avian neural crest: the role of neural plate-epidermal interactions. Development 121 : 525-538, 1995

33. Silver $\mathrm{MH}$, Kerns JM : Ultrastructure of neural fold fusion in chick embryos. Scanning Electron Microscope 2 : 209-216, 1978

34. Steinbok $P$ : Dysraphic lesions of the cervical spinal cord. Neurosurg Clin N Am 6 : 367-376, 1995

35. Steinbok P, Cochrane DD : The nature of congenital posterior cervical or cervicothoracic midline cutaneous mass lesions: report of eight cases. J Neurosurg $75:$ 206-212, 1991

36. Suneson A, Kalimo H : Myelocystocele with cerebellar heterotopia. J Neurosurg 51 : 392-396, 1979
37. Theveneau $E$, Mayor $R$ : Neural crest delamination and migration: from epithelium-to-mesenchyme transition to collective cell migration. Dev Biol 366 : 34-54, 2012

38. Tisdall MM, Hayward RD, Thompson DN : Congenital spinal dermal tract: how accurate is clinical and radiological evaluation? J Neurosurg Pediatr 15 : 651-656, 2015

39. van Aalst J, Beuls EA, Cornips EM, van Straaten HW, Boselie AFM, Rijkers K, et al. : The spinal dermal-sinus-like stalk. Childs Nerv Syst 25 : 191-197, 2009

40. van Aalst J, Beuls EA, Cornips EM, Vanormelingen L, Vandersteen M, Weber JW, et al. : Anatomy and surgery of the infected dermal sinus of the lower spine. Childs Nerv Syst 22 : 1307-1315, 2006

41. van Straaten HW, Jaskoll T, Rousseau AM, Terwindt-Rouwenhorst EA, Greenberg G, Shankar K, et al. : Raphe of the posterior neural tube in the chick embryo: its closure and reopening as studied in living embryos with a high definition light microscope. Dev Dyn 198 : 65-76, 1993

42. Wang KC, Yang HJ, Oh CW, Kim HJ, Cho BK : Spinal congenital dermal sinus--experience of 5 cases over a period of 10 years. J Korean Med Sci $8:$ 341-347, 1993

43. Wong ST, Kan A, Pang D : Limited dorsal spinal nondisjunctional disorders: Limited dorsal myeloschisis, congenital spinal dermal sinus tract, and mixed lesions in Di Rocco C, Pang D, Rutka JT (eds) : Textbook of Pediatric Neurosurgery, ed 1. Switzerland : Springer, 2020

44. Yamaguchi Y, Shinotsuka N, Nonomura K, Takemoto K, Kuida K, Yosida $\mathrm{H}$, et al. : Live imaging of apoptosis in a novel transgenic mouse highlights its role in neural tube closure. J Cell Biol 195 : 1047-1060, 2011 